\title{
Guidelines for the Treatment of Alcohol Problems
}

Prepared for the

Commonwealth Department of Health and Ageing

by

Fiona Shand, J ennifer Gates,

J ulia Fawcett, and Richard Mattick

National Drug and Alcohol Research Centre

(NDARC)

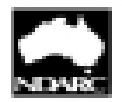

NATKONU DRLC AND

ACOHCL RESA WCHCENT: 
Commonwealth of Australia

ISBN 0642823839

This work is copyright. Apart from any use permitted under the Copyright Act 1968, no part may be reproduced by any process without prior written permission from the Commonwealth available from the Department of Communications, Information Technology and the Arts. Request and inquiries concerning reproduction and rights should be addressed to the Manager, Copyright Services, Info Access, GPO Box 1920, Canberra ACT 2601.

Publication Approval number 3379 (JN 8153)

Disclaimer

These Guidelines have been developed to assist health care workers. The diagnosis and treatment of alcohol problems require the consideration of an individual's particular circumstances by a qualified medical practitioner. These Guidelines are not a substitute for such advice, and should not be used to diagnose or prescribe treatment for any alcohol problem.

The views expressed in these Guidelines are not necessarily those of the Commonwealth or the National Drug and Alcohol Research Centre.

The Commonwealth and the National Drug and Alcohol Research Centre do not accept liability for any injury, illness, damage or loss incurred by any person arising from the use of or reliance on the information and advice that is provided in these Guidelines. 


\section{Contents}

List of Tables $\quad$ vi

List of Figures $\quad$ vi

Abbreviations vii

Acknowledgements $\quad x$

Executive Summary and Recommendations 1

Chapter 1: Introductory Comments 13

Purpose of the Guidelines $\quad 13$

Audience for the Guidelines $\quad 14$

$\begin{array}{ll}\text { Levels of Evidence } & 14\end{array}$

Chapter 2: Access to treatment: overcoming barriers 21

Chapter 3: Screening and assessment 25

$\begin{array}{ll}\text { The need for screening } & 25\end{array}$

In-depth assessment before more intensive treatment 33

Chapter 4: Patient-treatment matching 55

Chapter 5: Alcohol withdrawal management $\quad 59$

$\begin{array}{lr}\text { Alcohol withdrawal syndrome } & 59\end{array}$

$\begin{array}{ll}\text { Medical care for alcohol withdrawal complications } & 61\end{array}$

Home-based, outpatient and inpatient withdrawal management 69

$\begin{array}{ll}\text { Supervised medicated and non-medicated withdrawal management } & 73\end{array}$

Chapter 6: Post-withdrawal treatment setting 83

Who should receive residential treatment? $\quad 84$

Potential improvements to residential/therapeutic community care 86 
Chapter 8: Psychosocial interventions

Characteristics of effective clinicians

Counselling

Motivational interviewing

Cognitive-behavioural interventions

Skills training 108

Behavioural self-management

Cognitive restructuring

Cue exposure

Couples and family therapy

Self-guided materials

Standardised versus tailored treatment

Chapter 9: Relapse prevention

Identifying factors associated with relapse

Strategies for assessing relapse risk

Reducing the risk of relapse

Pharmacotherapies for relapse prevention

Chapter 10: Extended care

Treatment retention

Alcoholics Anonymous

Assertive outreach and structured aftercare 
Chapter 11: Interventions for specific client groups

Adolescents and young adults

Cognitive impairment 155

Comorbid disorders 158

Gender 161

Indigenous clients 164

Chapter 12: How to put it together

When there is no time available

Brief, one session, face-to-face interventions

Brief outpatient interventions

Longer multi-session outpatient interventions

Brief inpatient/residential withdrawal management 175

Day patient and inpatient/residential interventions

References 


\section{List of Tables}

$\begin{array}{ll}\text { Table } 1.1 \quad \text { Designation of levels of evidence } & 15\end{array}$

Table 3.1 Alcohol use disorders identification test screening instrument 29

Table 3.2 Levels of standard drinks 31

Table $3.3 \quad$ Issues to be covered in the initial clinical interview 36

Table 3.4 Severity of Alcohol Dependence Questionnaire Form-C (SADQ-C) 42

Table 3.5 The Severity Of Alcohol Dependence Data Questionnaire (SADD) 44

Table 3.6 Suggested scores on four measures of alcohol dependence to determine treatment goal and intensity

Table 5.1 Clinical Institute Withdrawal Assessment for Alcohol Revised (CIWA-Ar) 65

$\begin{array}{lll}\text { Table 5.2 } & \text { Alcohol Withdrawal Scale (AWS) } & 67\end{array}$

$\begin{array}{lll}\text { Table } 5.3 & \text { The Short Alcohol Withdrawal Scale (SAWS) } & 68\end{array}$

Table 5.4 Principles of supervised non-medicated alcohol $\begin{array}{ll}\text { withdrawal management. } & 74\end{array}$

$\begin{array}{lll}\text { Table } 5.5 & \text { Typical diazepam regime for alcohol withdrawal } & 77\end{array}$

$\begin{array}{lll}\text { Table 5.6 Diazepam regime for home -based alcohol withdrawal } & 77\end{array}$

$\begin{array}{lll}\text { Table 7.1 Common elements of a brief intervention } & 92\end{array}$

Table 8.1. Stages and steps of the Skilled Helper Model 101

$\begin{array}{lll}\text { Table } 8.2 & \text { The spirit of motivational interviewing } & 104\end{array}$

$\begin{array}{lll}\text { Table 8.3 The guiding principles of motivational interviewing } & 105\end{array}$

$\begin{array}{lll}\text { Table } 8.4 & \text { Standard units of alcohol } & 113\end{array}$

$\begin{array}{lll}\text { Table } 9.1 & \text { Summary of acamprosate and naltrexone characteristics } & 138\end{array}$

$\begin{array}{lll}\text { Table } 10.1 & \text { The twelve steps of Alcoholics Anonymous } & 146\end{array}$

\section{List of Figures}

$\begin{array}{lll}\text { Figure 5.1 Alcohol withdrawal syndrome progression } & 59\end{array}$

$\begin{array}{lll}\text { Figure } 8.1 & \text { The Skilled Helper Model } & 105\end{array}$

$\begin{array}{lll}\text { Figure } 12.1 & \text { Screening and intervention for alcohol problems } & 170\end{array}$

$\begin{array}{lll}\text { Figure } 12.2 & \text { Intensive treatment for alcohol dependence } & 171\end{array}$ 


\section{Abbreviations}

A\&D Alcohol and drug

$\mathrm{AA}$

Alcoholics Anonymous

ADCA

Alcohol and Other Drugs Council of Australia

AIHW

Australian Institute of Health and Welfare

ALT

Alanin aminotransferase

AOD

Alcohol and other drug

APQ

Alcohol Problems Questionnaire

AST

Aspartate

ATCA

Australasian Therapeutic Communities Association

AUDADIS-ADR Alcohol Use Disorder and Associated Disabilities Interview ScheduleAlcohol/Drug Revised

AUDIT Alcohol Use Disorders Identification Test

AWS

Alcohol Withdrawal Symptoms - rating scale

BAL Blood alcohol level

BCT Behavioural couples therapy

BDI Beck Depression Inventory

BI Brief Interventions

BMT Behavioural marital therapy

BSCT Behavioural self-control training

CBT Cognitive behavioural therapy

CDT Carbohydrate deficient transferrin

$\mathrm{CE} / \mathrm{CS} \quad$ Cue exposure with coping skills

CIDI Composite International Diagnostic Interview

CIWA-A Clinical Institute Withdrawal Assessment for Alcohol

CIWA-Ar Clinical Institute Withdrawal Assessment for Alcohol revised

CMP Client Matching Protocol

CNS Central nervous system

CRA Community reinforcement approach

DAS Depression, Anxiety and Stress Scale

DDD Drinks per drinking day 


$\begin{array}{ll}\text { DSM-IV } & \text { Diagnostic Statistics Manual-IV } \\ \text { DTs } & \text { Delirium tremens } \\ \text { DTCQ } & \text { Drug Taking Confidence Questionnaire } \\ \text { FRAMES } & \text { Feedback, responsibility, advice, menu, empathy, self-efficacy } \\ \text { GABA } & \text { Gamma-aminobutyric acid } \\ \text { GGT } & \text { Gamma-glutamyl transferase } \\ \text { GHB } & \text { Gamma- hydroxybutyrate acid } \\ \text { GHQ } & \text { General Health Questionnaire } \\ \text { ICD } & \text { International Classification of Diseases } \\ \text { IDTS } & \text { Inventory of Drug Taking Situations } \\ \text { KPMCP } & \text { Kaiser Permanente's Medical Care Program } \\ \text { MAST } & \text { Michigan Alcoholism Screening Test } \\ \text { MATCH } & \text { Matching Alcoholism Treatments to Client Heterogeneity } \\ \text { ME } & \text { Motivational enhancement } \\ \text { MET } & \text { Motivational enhancement therapy } \\ \text { mg } & \text { Milligrams } \\ \text { MI } & \text { Motivational interviewing } \\ \text { MM } & \text { Moderation management } \\ \text { MOCE } & \text { Moderation-oriented cue exposure } \\ \text { NAPS } & \text { Newcastle Alcohol-related Problems Scale } \\ \text { NCADA } & \text { National Campaign Against Drug Abuse } \\ \text { NDARC } & \text { National Drug and Alcohol Research Centre } \\ \text { NDRL } & \text { Non-directive reflective listening } \\ \text { NDS } & \text { National Drug Strategy } \\ \text { NDSHS } & \text { National Drug Strategy Household Survey } \\ \text { NAtional Health and Medical Research Council } \\ \text { NABional Institute on Alcohol Abuse and Alcoholism } \\ \text { NArinent }\end{array}$


PTSD

QAP

QFI

$\mathrm{RD}$

RFDQ

$\mathrm{RP}$

RTCQ

SADD

SADQ-C

SAWS

$\mathrm{SC}$

SCAN

SF-12

SNAP

SSRI

TC

TGA

TLFB

TrEAT

TSF

TTM

TWEAK

URICA

WAIS-III

WHO

WKS
Post Traumatic Stress Disorder

Quality Assurance Project

Quantity-frequency index

Retrospective diary

Reasons for Drinking Questionnaire

Relapse prevention

Readiness to Change Questionnaire

Short Alcohol Dependence Data

Severity of Alcohol Dependence Questionnaire

Short Alcohol Withdrawal Scale

Standard care

Schedules for Clinical Assessment in Neuropsychiatry

Short Form 12

Smoking, Nutrition, Alcohol and Physical Activity

Selective serotonin reuptake inhibitors

Therapeutic Community

Therapeutic Goods Administration

Timeline Followback Method

Treatment for Early Alcohol Treatment

Twelve step facilitation

Transtheoretical model

Tolerance, worried, eye opener, amnesia, cut down

University of Rhode Island Change Assessment

Wechsler Adult Intelligence Scale III

World Health Organization

Wernicke-Korsakoff's Syndrome 


\section{Acknowledgements}

The Management of Alcohol Dependence Project Expert Panel consisted of:

Professor Steven Allsop

Dr Jenny Bowman

Dr Charlotte de Crespigny

Mr Barry Evans

Associate Professor Aidan Foy

Dr Paul Haber

Professor Mark Harris

Ms Leanne Keen

Dr Alison Marsh

Ms Kathryn Modystack

Professor Philip Morris

Ms Jan Parr

Professor Robyn Richmond

Dr Alison Ritter

Dr Anthony Shakeshaft

Dr Moira Sim

Dr John Stanhope

Professor Greg Whelan

Dr Alex Wodak

\section{Professor Nick Zwar}

Special thanks to the following panel members for their extra contributions: Steve Allsop, Charlotte de Crespigny, Paul Haber, Phillip Morris, Jan Parr, Anthony Shakeshaft, and Moira Sim. Thanks to Sophie Reid for her assistance with information on compliance therapy, and to Eva Congreve for her assistance in locating books and journals. Our gratitude to Heather Proudfoot for preliminary editing, to Maree Teesson for her valuable advice early in the project, to Yvonne Bonomo for reviewing the section on interventions for adolescents, and to Julie Watkinson for her considerable work in reviewing drafts of the guidelines. 


\section{Executive Summary and Recommendations}

\section{Introduction}

These guidelines are based on a review of the evidence about the effectiveness of treatments, and on the clinical experience of an expert panel.

The aim of the guidelines is to provide evidence that guides treatment, education and professional development. It is not our aim to dictate treatments.

These guidelines are intended for all health workers and medical practitioners who come into contact with dependent or problem drinkers. Summary guidelines are available in mid-2003.

Problem drinking affects a broad cross section of Australia's population, with impacts on health, social, employment, and economic outcomes. However some groups face barriers to treatment, namely lower socioeconomic groups, those living in rural and remote communities, Indigenous Australians, prisoner inmates, those with comorbid mental health problems, and older people.

SCREENING

STRENGTH OF

RECOMMENDATION

Screening for risky and high risk alcohol consumption should Strong be widely implemented in general practice, hospitals, community health care, and workplaces.

The AUDIT, the Quantity Frequency Index or the Retrospective Diary Strong are recommended for screening in the above settings.

The T-ACE and the TWEAK are recommended for screening risky Strong drinking in pregnant women.

\section{ASSESSMENT}

Assessment for more intensive intervention should be conducted in a semi-structured, narrative style, with strategic use of structured questionnaires such as the SADD or the SADC-Q.

The length of the assessment process needs to be balanced with Strong retaining the client in treatment. Therefore, clinicians need to make Strong decisions about which aspects of assessment are essential. Key topics are the client's consumption of alcohol, level of alcohol dependence, cognitive functioning, psychological comorbidity, family situation, physical well-being, and readiness for change.

Assessment should lead to a clear, mutually-acceptable treatment plan Strong that structures a specific intervention to meet the needs of the individual.

Readiness to change may be best assessed via direct questioning.

Moderate 
Where possible and with the client's permission, family members

Moderate

should be involved in the assessment process.

Assessment for anxiety and depressive symptoms and disorders

Strong

should be routine.

\section{PATIENTT-TREATMENT MATCHING}

The intensity of interventions should vary, with clients with more

Moderate

severe problems receiving more intensive treatments.

Co-morbid psychological disorders should be taken into account when

Moderate

assigning patients to treatment, particulanly when pharmacotherapies

are being considered as a relapse prevention strategy.

Where possible, clients should be offered a choice of interventions.

Moderate

The pros and cons of abstinence versus moderated drinking should

be considered in light of the client's goals, their level of dependence,

their physical health and their relapse history.

\section{WITHDRAWAL MANAGEMENT}

Patient experiencing withdrawal complications such as seizures,

Strong

hallucinations, delinum and delinum tremens should be monitored

carefully and regularly using a withdrawal rating scale.

Patients at risk of Wemicke-Korsakoff's syndrome should be treated

Strong

with 100mg of intramuscular or oral thiamine before any glucose intake.

Patient withdrawing from alcohol should be monitored with

Strong

a withdrawal rating scale. The CIWA-Ar is the recommended scale.

If the CIWA-Ar scale is unavailable then the AWS should be used.

Fair

Although the AWS is widely used in Australia, it is not validated.

Home-based withdrawal management is recommended for patients

with mild to moderate withdrawals who have a support network,

no known co-existing medical or psychiatric illness, and no history

of withdrawal complications.

Outpatient withdrawal management is appropriate for patients with

mild to moderate withdrawals who have no history of severe withdrawal,

Strong

and no known co-existing medical or psychiatric illness.

Inpatient withdrawal management is required for people who have

severe withdrawal complications, a history of withdrawal complications,

and/or a known co-existing medical or psychiatric history.

Supervised non-medicated withdrawal management is appropriate only for people with mild to moderate alcohol withdrawal, and for those who have no known coexisting medical or psychiatric illnesses.

Strong 
A supervised medicated withdrawal is required for people who are Strong at risk of or suffer from alcohol withdrawal complications.

Diazepam is recommended as the "gold-standard" and first-line treatment for alcohol withdrawal symptoms.

Anti-convulsant medications are recommended for patients who take Moderate them on a regular basis before admission, and with patients who have epilepsy related to alcohol withdrawal.

Haloperidol (a major tranquilliser) is recommended for use with patients experiencing hallucinations and/or paranoid symptoms where diazepam is not effective in reducing or alleviating the hallucinations and paranoid symptoms.

100mg of intramuscular or oral thiamine should be administered before any glucose intake to any patient at risk of alcohol withdrawal complications.

Multi-vitamins and fluids should be given to any patient withdrawing from alcohol.

Moderate POST-WITHDRAWALTREATMENT SEITING

Decisions about treatment setting should be based on the client's Strong treatment goals, preferences, severity of dependence, the presence of comorbid disorders, cognitive and social functioning, relapse history, and social circumstances.

Residential programs should aim to increase retention in treatment by running structured, interactive induction sessions addressing the problems of staying in treatment and other client concerns, by using motivational interviewing techniques, and by strengthening the client's involvement in treatment and therapeutic alliances.

Residential programs should be modified to meet the needs Moderate of particular groups, for example those with comorbid disonders, those with childcare responsibilities, and the homeless.

\section{BRIEF INTERVENTIONS}

Brief interventions should be routine practice in general practice Strong settings, general and emergency hospital wards, and community counselling centres.

Brief interventions should consist of the six components of the FRAMES acronym: feedback, responsibility, advice, menu, empathy and self-efficacy.

Moderate 
Where brief interventions are not successful in reducing alcohol

Strong

consumption, a more intensive treatment should be offered to the client.

Health care professionals who work in general practice settings,

Strong general and emergency hospital wards, and community counselling services should be trained to deliver brief interventions.

\section{PSYCHOSOCIAL INTERVENTIONS}

Since clinician characteristics influence treatment outcomes, it is recommended that clinicians receive interpersonal skills training, focusing particularly on empathy.

An organised approach to treatment is most effective, supported by Moderate careful case and progress notes.

General counselling skills should form the basis of

a therapeutic relationship.

General counselling skills should be supported by more specific

Strong techniques aimed at initiating a change in behaviour.

Motivational interviewing is recommended as a treatment intervention.

Strong

Motivation is recommended particularly for patients who are

Moderate ambivalent about changing their behaviour.

Personalising information about the adverse health effects of nisky Strong drinking is recommended as a strategy to increase motivation to change.

The various interventions that fall under the umbrella of cognitive Strong behavioural interventions should be implemented in treatment settings.

Skills training should be offered to clients who lack the relevant skills. Strong

Problem solving skills training, assertiveness skills training, Strong communication skills training, drink refusal skills training, and relaxation and stress management skills trainings are recommended for use with risky and dependent drinkers.

Although appropriate for individual settings, skills should be Moderate implemented in group settings to allow for role-play and modelling.

Skills training is recommended for patients who have a high Strong nisk of relapse.

Behavioural self-management should be offered to clients with a goal Strong of moderation, and to those who are extremely unlikely to stop drinking. 
The client should be introduced to the National Health and Medical

Strong

Research Council's Alcohol Guidelines.

Clients should be taught the concept of a standard drink to assist

Strong

in monitoring levels of consumption.

Cognitive restructuring procedures are recommended as part of more

Strong

comprehensive interventions that rely on providing the client with specific skills.

Cognitive restructuring is not recommended for patients with cognitive deficits.

Strong

Cognitive restructuring should be practised throughout the

Strong

treatment process.

Cue exposure should consist of six to 12 sessions of 50 - 90 minutes.

Moderate

Cue exposure should only be offered by treatment specialists who

Strong

have appropriate training in the strategy.

Behavioural couples therapy should emphasise that drinking is the problem, and focus on drinking behaviour as the problem which needs to be fixed.

Behavioural couples therapy should not address entrenched

relationship problems or be used to counteract violence.

Behavioural couples therapy is recommended for couples with

Fair moderate to low problems in their relationship, couples who are living together, are at least high school educated and are employed.

Clinicians who deliver behavioural couples therapy should be suitably trained in the delivery of this intervention.

The interventions delivered should be supported by empirical research.

Strong

Self-help manuals should be made readily available to the general Strong public to assist drinkers who wish to cease or cut-down drinking without the aid of professionals.

Self-help materials are most appropriate for risky drinkers rather than those suffering from alcohol dependence.

Moderate

Self-help manuals should be modified to suit people from non-English

Strong

speaking backgrounds and those with a low reading age.

Computer and intemet based self-help materials should continue to be developed, as they may provide helping materials to a population of problem drinkers who may not ordinarily access treatment. 
Treatment intervention procedures should be clearly specified

Strong

in a written form.

Clinicians should be adequately trained in the procedures involved.

Strong

RELAPSE PREVENTION

Psychosocial relapse prevention strategies are recommended for use

with all moderate to severely alcohol dependent clients.

Psychosocial relapse prevention is best delivered after acute

Moderate

withdrawal symptoms have subsided.

High risk situations should be assessed for each client, along with

other risk factors such as cravings, disease beliefs, and coping skills.

Acamprosate is recommended as an adjunct to psychosocial relapse

Strong prevention for moderate to severely alcohol dependent clients,

with the exception of those contraindicated as per table 9.1.

Naltrexone is recommended as an adjunct to psychosocial relapse prevention for moderate to severely alcohol dependent clients, with the exception of those contraindicated as per table 9.1.

Patients who are opioid dependent should not be prescribed Strong naltrexone. All patients should be warned of the potential for opioid withdrawal syndrome if any form of opiates is taken, i.e. heroin, methadone, and opioid analgesics.

For some patients, GP management to accompany acamprosate Moderate and naltrexone will be sufficient. More severely dependent clients and chronic relapsers will need more intensive psychosocial interventions. Thus, psychosocial treatment should be tailored to the needs of the patient and should include relapse prevention strategies.

Acamprosate and naltrexone should be started within one week Moderate of detoxification.

Acamprosate and naltrexone are usually taken for three to six months, Moderate and in some cases up to 12 months. Optimum treatment duration has not been established.

Many patients find it difficult to comply with a medication program.

This issue should be addressed with compliance therapy which is based on motivational interviewing and cognitive-behavioural strategies. 
Attention should be given to the client's retention in treatment and Strong social support networks.

Aftercare should be structured and assertive, with follow-up of missed appointments.

Strong

Clients who show signs of dependence on alcohol should be made Moderate aware of the service offered by meetings of Alcoholics Anonymous (AA). Mandated AA participation is not recommended.

Conventional AA meetings should not be viewed as treatment for Moderate alcohol dependence.

TREATIMENT ISSUES FOR SPECIFIC GROUPS

\section{ADOLESCENTS}

Given the limitations of diagnostic criteria for alcohol use disorders

with adolescents, a flexible approach to diagnosis and treatment is needed. Whilst some young people may not meet strict diagnostic criteria for alcohol dependence or abuse, this should not be a bamier to treatment.

Clinicians should focus on building and maintaining a relationship Strong with younger clients through outreach, crisis intervention and harm reduction activities, and the use of an empathetic, non-judgemental, developmental approach to counselling.

Brief and motivational interventions help to reduce alcohol consumption among adolescent heavy or binge drinkers, across a range of settings.

Younger drinkers who require more intensive intervention may Strong have different treatment needs to older drinkers. These needs should start to be identified during the assessment phase, and may include crisis intervention, assertive outreach, and building social support networks.

The recommended psychological treatment for young people Moderate is cognitive behavioural therapy. Other potentially useful treatments include social skills training, family therapy, therapeutic support groups, and interpersonal therapy.

\section{COGNITIVELY IMIPAIRED CLIENTS}

A brief assessment of cognitive functioning should be a routine part of assessment upon treatment entry.

More detailed assessment should be camied out where brief assessment 


\section{TREATMENT ISSUES FOR SPECIFIC GROUPS}

STRENGTH OF

RECOMMENDATION

\section{COGNITIVELY IMPAIRED CLIENTS}

Where cognitive impairment is confirmed, information presented

Strong

to patients should be concrete and patients should be given

opportunities to practise behaviours taught during treatment sessions.

Cognitively impaired patients should be engaged in treatment

by the clinician by:

- providing information about treatment

- discussing different treatment options

- establishing a positive relationship

- maintaining contact with the client

The clinician should frequently check that the client understands what

is being said, and that the clinician understands what the client is saying.

The possibility of improvement in cognitive functioning should be taken into account by allowing a sufficient period of abstinence

from alcohol to elapse before finalising treatment planning.

Cognitively impaired patients should be taught relapse

Moderate

prevention strategies.

\section{COMORBID DISORDERS}

The AUDIT is recommended for screening psychiatric populations.

Strong

Assessment for comorbid disorders should take place once the

Strong

client's withdrawal syndrome has diminished, since some anxiety

and depressive symptoms may abate once alcohol consumption

is reduced or ceased.

Comorbid mood and anxiety disorders which do not abate

after alcohol withdrawal is complete should be treated with integrated/concurrent CBT for the cormorbid disorder.

Alcohol dependent patients with comorbid anxiety should be considered for treatment with buspirone, depending on the severity and nature of their symptoms.

Alcohol dependent patients with major depression should

Fair

be considered for treatment with desipramine, depending on the severity and nature of their symptoms.

Schizophrenic patients who are risky drinkers should be considered for treatment with clozapine or nisperidone, depending on the severity and nature of their symptoms. 


\section{TREATMENT ISSUES FOR SPECIFIC GROUPS}

STRENGTH OF

RECOMMENDATION

\section{COMORBID DISORDERS}

Alcohol dependent patients with antisocial personality disorder Fair should be considered for treatment with nortriptyline, depending on the severity and nature of their symptoms.

\section{GENDER}

Treatment providers need to be aware of and sensitive to issues Strong particular to women with alcohol problems.

Service providers should try to improve the recruitment of women to their services by improving outreach, setting up referral networks, advertising facilities specifically targeting women, and providing more detailed information about services. This would increase women's awareness of treatment services available, and may decrease any reservations or fears about entering treatment.

All clients should be provided with a safe therapeutic environment, Strong as the prevalence of physical and sexual abuse is high, particularly among women with alcohol problems.

Treatment agencies should offer all clients the opportunity to receive information about, and address health and other issues such as depression, anxiety and sexual and physical assault.

Women should be referred to other specialist services where necessary and appropriate.

Moderate

Where possible, patients should be given the option of having a clinician of the same or opposite gender, as preferred.

\section{INDIGENOUS CLIENTS}

Relapse among Indigenous clients who have completed treatment Moderate is likely to be high when clients return to a poor socio-economic situation. Treatment services therefore need to be supported by effective, culturally appropriate interventions at the population level.

Clients may present to health care clinics with minor complaints without volunteering information about alcohol consumption. Strong Carefully worded questions about the frequency of heavy drinking and average daily consumption can help to identify risky drinking.

Cultural sensitivity should not be confused with treatment efficacy. Strong For example, brief interventions may still be effective with Indigenous Moderate drinkers, but ways of communicating and knowledge of issues such as gender roles are important issues in their implementation. 


\begin{tabular}{l|l}
\hline TREATMENT ISSUES FOR SPECIFIC GROUPS & $\begin{array}{c}\text { STRENGTH OF } \\
\text { RECOMMENDATION }\end{array}$ \\
\hline INDIGENOUS CLIENTS & \\
\hline $\begin{array}{l}\text { Increasing compliance with medications such as acamprosate } \\
\text { and naltrexone for Indigenous clients may entail addressing } \\
\text { a slightly different set of issues, which will in turn depend on the } \\
\text { client's cultural environment. }\end{array}$ & Moderate \\
\hline $\begin{array}{l}\text { There is great potential for opportunistic intervention by medical } \\
\text { staff in hospitals, as well as in other settings, such as in sobering } \\
\text { up centres, workplaces, correctional facilities, and community }\end{array}$ & Strong \\
health services. & \\
\hline $\begin{array}{l}\text { The services available for Indigenous clients need to provide } \\
\text { a greater quality and diversity of treatment options. }\end{array}$ & Strong \\
\hline $\begin{array}{l}\text { Health care practitioners should seek to keep clients engaged } \\
\text { in treatment, whether or not the drinking behaviour changes. }\end{array}$ & Strong \\
In the meantime, support can be offered to the drinker's family \\
and community.
\end{tabular}


Chapter 1: Introductory comments 


\section{Chapter 1: Introductory comments}

In 1993, the National Drug and Alcohol Research Centre (NDARC) completed a monograph on behalf of the National Campaign Against Drug Abuse (NCADA), entitled "An outline for the management of alcohol problems: Quality assurance project for the management of alcohol dependence" (QAP). ${ }^{[1]}$ The primary aim of the project was to advise drug and alcohol treatment personnel in Australia on the current knowledge about the effectiveness of various techniques for assisting people who are risky drinkers or who exhibit a dependence on alcohol.

The Commonwealth, as part of the National Drug Strategy, has commissioned NDARC to develop updated guidelines for the treatment of alcohol problems. Since 1993 research has been undertaken for a range of treatments that at the time were relatively new or unavailable in Australia.

These guidelines are based on a review of the evidence for treatment efficacy, and upon the clinical experience of the expert panel. In developing the guidelines, we have relied where possible on evidence from well-designed research studies. Where this evidence was not available, recommendations are based upon appropriate clinical experience. There is a "summary of evidence" box at the end of each section. From this, readers can determine the strength of the evidence upon which the recommendations that follow are based.

As with the 1993 guidelines, our intention is to provide evidence that guides rather than dictates treatment interventions, education and professional development. Current research techniques do not allow us to rule in or out various treatment methods: they do, however, provide a sound level of evidence about what is effective in a research setting. In practice, many other factors impact upon the effectiveness of treatment (e.g. resource availability, patients' comorbid disorders, clinical skills). However, it is reasonable to expect that treatments possess some evidence of efficacy before they are supported in anything other than an experimental way.

There are a number of treatment guidelines for treating people with alcohol problems. This document is not intended to replace existing guidelines, rather, it provides a broad reference to a range of treatment options. Where it is appropriate and relevant, we refer practitioners to existing guidelines for more detail about the use of particular interventions. In other instances, we have integrated - with acknowledgement - existing recommendations into our own.

\section{Purpose of the guidelines}

The primary aim of the current guidelines is to provide up-to-date, evidence-based information to clinicians on the available treatments for people with alcohol problems. Up-to-date information is required because of the size and importance of the health burden created by alcohol problems, and the variations in practice for the treatment of alcohol dependence. Variation per se is not problematic. Different treatments are appropriate for different clients. However, variation in the quality of care remains an issue. In some instances, this is a resource and equity issue rather than an issue of knowledge. These guidelines are aimed at the latter. 


\section{Audience for the guidelines}

This comprehensive version of the guidelines is intended for clinicians who want a full review of the treatment options for alcohol problems. Specific, summarised guidelines are also available (in mid-2003) for general practitioners, drug and alcohol workers, general hospital workers, and consumers. Throughout this document we use the terms "clinician" or "health care worker" to refer to any of the above professionals.

\section{Development of the guidelines}

The guidelines are based on a review of the available evidence of efficacy (the Treatment of Alcohol Problems: A Review of the Evidence) and the knowledge of an expert panel convened to develop the guidelines. Members of the expert panel are listed in the preliminary pages. The procedure used to identify research has involved searching relevant databases for published clinical trials, hand searching journals, searching website bibliographies, canvassing for unpublished research, and contact with major research centres for unpublished research and other relevant guidelines. Databases searched include Medline, Psychinfo, Cochrane Database of Systematic Reviews, the National Clearinghouse for Alcohol and Drug Information, Evidence Based Medicine Reviews, and the Alcohol and Other Drug Council of Australia's (ADCA) Drug Database.

\section{Levels of evidence and strength of recommendations}

The National Health and Medical Research Council levels of evidence hierarchy (Table 1.1) was used to classify the quality of the evidence available in each treatment area. ${ }^{[2]}$ The preferred level of evidence was a systematic review of all relevant randomised controlled trials. Overall, the quality of evidence available was high: meta-analyses ${ }^{1}$ have been completed for most of the major treatment modalities. We have therefore opted to use these existing analyses.

For each treatment modality we have included the findings from meta-analytic reviews with a narrative review of other relevant evidence, typically randomised controlled trials. Quality evidence is scant for the effectiveness of treatment of specific sub-groups: indigenous clients, adolescents and those with comorbid mental disorders. For these areas, we have reviewed clinical trials where available, or otherwise relied on expert opinion. Similarly, while the residential/non-residential rehabilitation debate has been discussed and researched at length, little has been done to clarify which components of residential rehabilitation treatment work best, or for which clients residential treatment is recommended.

A randomised controlled trial refers to a study that has at least one treatment group and a control group, usually placebo or no treatment. The study uses outcome measures before and after treatment, and randomly assigns participants to the groups. Some trials, normally those testing medications, also use a double blind where neither the participants nor the researcher know who is receiving which treatment, or a single blind design where either the participants or the researcher does not know who is receiving which treatment. Controlled trials allow the researcher to conclude with a degree of certainty whether or not the treatment being tested is more effective than no treatment. In field research with patients, this ideal design is not always possible because of ethical concerns. However it is still possible to draw conclusions from some of these quasi-experimental

1 Meta analysis is a statistical technique which combines a number of single trials to increase the overall power and certainty of outcomes. 
designs, provided the correct statistical analysis is used to control for confounding variables. The conclusions drawn though might be more tentative.

\section{TABLE 1.1: DESIGNATION OF LEVELS OF EVIDENCE}

\section{LEVEL OF EVIDENCE}

I

II

III-1

III-2

III-3

\section{STUDY DESIGN}

Evidence obtained from a systematic review of all relevant randomised controlled trials.

Evidence obtained from at least one properly designed randomised controlled trial.

Evidence obtained from well-designed pseudo-randomised controlled trials (alternate allocation or some other method).

Evidence obtained from comparative studies (including systematic reviews of such studies) with concurrent controls and allocation not randomised, cohort studies, case-control studies, or interrupted time series with a control group.

Evidence obtained from comparative studies with historical control, two or more single arm studies, or interupted time series without a parallel control group.

IV

Evidence obtained from case series, either post-test or pre-test/post-test.

Source: "How to use the evidence: assessment and application of scientific evidence", National Health and Medical Research Council, 2000. Reproduced with permission. ${ }^{[2]}$

The strength of recommendation is based primarily on the evidence presented for the technique in question, combined with clinical expertise. Three levels are used:

\section{STRENGTH DESCRIPTOR \\ OF RECOMMENDATION}

Strong

Moderate

Fair
The recommendation is supported by at least level II research and expert clinical opinion.

The recommendation is supported by at least level III research and expert clinical opinion.

The recommendation is based on expert clinical opinion. 
Although experimental research evidence is the most appropriate way to determine the relative efficacy of one treatment against another, the effects seen in such trials might be diluted when the interventions are applied in normal clinical settings. Most trials examine the effects of interventions under highly controlled and ideal conditions. Attention should also be given to more pragmatic trials where interventions found to be efficacious in highly controlled trials are tested for their effectiveness in real-life settings. Loss of effect can result from factors associated with the realities of health care delivery, such as the training and experience of clinicians, the faithfulness with which the intervention is delivered, and the time and resources that are available to implement the intervention. These problems are present in all areas of health care, although they are likely to be more marked in the case of non-pharmacological and non-proprietary methods of intervention. ${ }^{[3]}$ This commentary represents a clear caution about the adequacy of the implementation of interventions. The guidelines provide a summary of treatment procedures that are advocated for use in interventions, from relatively brief interventions through to more intensive approaches to the management of risky drinkers and alcohol dependent individuals, respectively.

Despite the coverage of brief interventions, the interventions that are designed to assist people with serious drinking problems remain the major focus of this document. Brief interventions are an important part of the overall approach to alcohol problems in Australia, but they should not be expected to replace specialist interventions that deal with chronic and severe cases. Programs for alcohol dependent patients need to vary in intensity and length according to the degrees of dependency and other problems in those drinkers.

A distinction is drawn between two types of intervention: proactive and reactive. Proactive interventions are those that should routinely occur when a person is recognised as a risky drinker by a health care worker, even where drinking is not a focus of the examination. Thus, these proactive interventions occur when the risky drinker has not specifically sought help for alcohol problems. For a proactive intervention to occur, the health care worker needs to screen clients routinely for alcohol problems to detect risky drinkers, and offer them an intervention aimed to reduce alcohol consumption. The methods for proactive interventions are covered in Chapter 3 : Screening and assessment and Chapter 7: Brief interventions. In contrast, reactive interventions are those that are conducted when the drinker directly seeks help for an alcohol problem. The assessment and treatment plan involved in this intervention is likely to be longer than in the proactive case. Most of this document is devoted to this type of intervention. Where appropriate, clients can be referred from a brief, proactive intervention to an intensive intervention.

\section{What the guidelines cover}

These guidelines are complementary to existing practical guidelines for the treatment of alcohol problems and are to help clinicians make decisions about the type of interventions that should be made available in their practice. A range of appropriate treatment procedures is described in this monograph so that clinicians can select those approaches that match the setting and client type with which they deal. 
The treatment guidelines here provide a summary of what is supported by current research and specialist opinion. Clinical practice must change with advances in the field. It is up to informed individual clinicians to use the guidelines to guide but not to limit treatment needed for the circumstances of individual patients. The recommendations are outlines for treatment approaches, not mandatory and inflexible rules for intervention. However, it is inappropriate for clinicians in Australia to continue using treatment approaches of uncertain efficacy when there are procedures for which there is reasonable evidence of effectiveness available. It is the responsibility of individual drug and alcohol workers and counsellors as well as the government systems which support treatment provision to ensure that the treatments made available are those believed to be the most effective.

Interventions not in these guidelines either had no research supporting their effectiveness, based on the review of the literature, or were deemed irrelevant because of undeveloped research, or because the intervention concerned was not easily implemented. The information presented in these guidelines should allow interested groups to design an overall intervention by selecting from the procedures advocated. These guidelines do not attempt to provide information about systems of treatment delivery, which is a policy decision that relates to the needs, resources and structure of health care within jurisdictions.

Finally, it is recognised that some people with alcohol problems change without formal help or intervention. A complete account of alcohol use involves general societal factors, such as the availability of alcohol, societal pressure to drink or not drink, prohibitions on permissibility of use and restrictions on the age of use. At a more personal level, factors such as peer pressure, familial and parental drinking patterns, as well as early learning experiences, life events, initial drinking experiences and genetic predisposition are involved. It is not surprising then that there are many influences outside the formal treatment system that affect drinking behaviour. Many of these influences can also be directly manipulated within a formal treatment setting to take advantage of this effect on drinking behaviour, but it remains true that formal treatment will affect the drinking of only a proportion of the risky and problem drinkers in our society. Even so, there is a need for a formal treatment approach based on reasonable evidence. 
Chapter 2: Access to treatment: overcoming barriers 


\section{Chapter 2: Access to treatment: overcoming barniers}

\section{CHAPTER AIM}

The aim of this chapter is to identify barriers that drinkers may encounter in seeking treatment.

Many barriers to treatment are equity-based and systemic. For example, lower socioeconomic groups are less likely to be offered brief interventions. ${ }^{[4]}$ Remote Indigenous communities often have very limited access to treatment resources. ${ }^{[5]}$ Types of barriers include:

- Lower socioeconomic status.

- Lack of resources in rural areas, particularly within Indigenous communities

- Homelessness

- Comorbid psychological disorders, especially psychosis and schizophrenia

- Systemic barriers within the health care system (for example, Medicare rebate structure) which influence health professionals' behaviour

- Lack of awareness on the part of health professionals, and/or unwillingness to "interfere"

Some dependent drinkers face a number of these barriers. Those thought to be most at risk of harm include young adults, older males, pregnant women, Indigenous people, prisoners, and people with a comorbid psychological disorder.

\section{Lower socioeconomic groups, rural and remote communities}

Homeless people are included in this group, many of whom also have a comorbid psychiatric disonder. Often current health-care structures do not reach many of these people, let alone cater for their complex needs. Assertive outreach and follow-up is required to locate, treat and retain these clients in treatment.

In remote communities, health care workers may be faced with great demands upon their skills and resources to deal with alcohol related problems because of a lack of specialist services in the local area. Many dependent drinkers have family responsibilities which prevent them from travelling to seek specialist treatment. Thus, general practitioners and nursing staff must attempt to meet the varied needs of these patients.

\section{Indigenous Australians}

Although Indigenous Australians are less likely to consume alcohol than non-Indigenous Australians, many of those who consume alcohol drink at risky levels. ${ }^{[6]}$ In some Indigenous communities the key barriers include:

- Lack of a strong relationship with health care workers and a real or perceived lack of cultural sensitivity

- Restricted health services and opportunities for health education

- Lack of time and resources to deal with anything other than the presenting problem 
- Unwillingness by health care workers to raise the topic of risky drinking

- Awareness that the environment to which the patient is returning does not support nondrinking and a subsequent sense of hopelessness on the part of health care workers

- Some patients may become annoyed when alcohol is raised as an issue in addition to their presenting problem

- The severity and complexity of the illness and problems with which patients present

Annual use of hospital outpatients and emergency rooms is much higher in the indigenous than the non-indigenous population (8 percent vs 2.5 percent). Indigenous people are three times more likely to use outpatients than to go to a doctor's practice. This points to the potential for interventions by medical staff working in hospital settings. Other opportunities for intervention include workplaces, sobering-up centres, and correction facilities. ${ }^{[5]}$

Interventions based around the principles described in Chapter 7: Brief interventions can be used in many of these settings as a first step towards changing risky drinking behaviour. For more information, see Chapter 11: Interventions for specific client groups.

\section{Prison inmates}

The main barriers to treatment for inmates include under-diagnosis, limited access to health services, and non-screening of risk behaviours.

\section{Comorbidity}

Mental health problems commonly co-occur with alcohol use disorders. Some mental disorders, such as schizophrenia, are correlated with homelessness, thus making this population even more difficult to reach and to keep in treatment. Other mental health problems such as personality disorders complicate treatment so much that some providers are reluctant to accept these clients into treatment. Still other problems such as mood and anxiety disorders are more prevalent among women who may have difficulties attending treatment because of family responsibilities.

\section{Older people}

Under-diagnosis appears to be the key barrier to treatment for older patients. For instance, hospital workers are significantly less likely to identify alcohol-related problems in an older patient than in a younger patient. [?]. 
Chapter 3: Screening and assessment 


\section{Chapter 3: Screening and assessment}

\section{Chapter Aim}

The aim of this chapter is to guide screening which might take place prior to a brief and/or early intervention for those patients who may be drinking at risky levels, or have risky patterns of consumption.

\section{The need for screening}

There is strong evidence for the cost-effectiveness of screening and early intervention in primary care settings. ${ }^{[8,9]}$ Given the pervasiveness of risky alcohol consumption in Australia and the seriousness of the health consequences of risky drinking, detection of risky alcohol consumption through routine screening should be conducted in all health care settings. This activity must be followed by a time-limited intervention aimed at reducing consumption for those with risky levels or patterns of drinking.

Screening should be conducted in settings where the prevalence of risky drinkers is likely to be highest and where detection will have the greatest salience for both the health care worker and the drinker. The following settings are appropriate for screening. Their order reflects their probable effect, with medical settings most likely to show a high rate of identification, followed by workplace and other health care settings. The settings include the following sites:

\section{Medical practices.}

Detection and brief intervention activities should be encouraged in general and specialist medical practices. Barriers to implementing brief interventions in general practice settings are described in Chapter 7: Brief interventions. ${ }^{[10]}$ Because of their role in primary health care and their high rate of contact with the general public, general practitioners are ideally placed to detect and offer patients help with drug and alcohol problems. ${ }^{[1]}$

\section{General hospitals.}

In all general hospitals routine screening procedures for risky alcohol consumption among inpatients and outpatients, and procedures for appropriate intervention should be in place. These procedures should be followed by brief intervention and referral as necessary. This may include a letter to the referring GP giving feedback about the level of risky consumption and advising the need for followup of the patient. Hospital accreditation should assess whether these screening procedures are in place and are followed.

\section{The workplace.}

Young male drinkers, who are less likely to attend primary care settings, may be screened in the workplace. Detection of risky consumption should be a part of any routine health evaluation in the workplace. Such screening and brief intervention increase the health and safety of workers, and limit hazards and accidents in the workplace. There is evidence of high rates of problem drinking in some of these settings suggesting that the workplace is a suitable venue for detection of risky drinking and intervention. ${ }^{[12]}$ 


\section{Welfare and general counselling services.}

Although not an obvious setting for detection activities, screening in welfare and counselling services offers the opportunity of referral for intervention, and potentially a better outcome for the clients of these services. It is likely in a significant proportion of cases that risky alcohol intake has contributed to the presenting problem.

In all of these settings, there is a need to develop a structure where screening can occur in a routine way, thereby increasing the likelihood that it will become and will remain a part of the normal activities. There are significant barriers to the widespread adoption of screening and intervention procedures. For instance, there are few incentives and some disincentives to primary health care workers and others becoming involved in screening activities [13]. See Chapter 7, Brief Interventions.

A number of initiatives to deal with these barriers are being or will be undertaken as part of the Smoking, Nutrition, Alcohol and Physical Activity (SNAP) Framework for General Practice. ${ }^{[4]}$

\section{QUESTIONNAIRE APPROACHESTO SCREENING}

The approaches used to detect people with risky drinking patterns vary considerably across settings. For specialist drug and alcohol agencies, detecting risky drinkers is less of an issue because their clients are likely to declare their drinking problem when they present for assistance. For general practitioners and other general health care workers, however, screening is a major consideration. There are a number of methods for detecting risky drinkers, including asking the person about their alcohol consumption, using screening questionnaires, or clinical examinations to assess for the presence of signs and symptoms of risky use of alcohol, and biological markers of excessive consumption. One established method for detecting people with risky drinking habits is the use of a standard questionnaire. Many questionnaires have been designed to screen for alcohol dependence, but only a few have been devised specifically to detect risky drinkers who may be non-dependent.

The questionnaires described below do not represent an exhaustive list of the available instruments. See the National Drug Strategy publication, "Review of diagnostic screening instruments for alcohol and other drug use and other psychiatric disorders", for a description of instruments available for screening for risky alcohol and other drug consumption. ${ }^{[14]}$

\section{DETECTING RISKY ALCOHOL CONSUMPTION}

The Alcohol Use Disorders Identification Test (AUDIT) was designed to detect people with risky alcohol consumption (Table 3.1). ${ }^{[15]}$ The AUDIT is comprised of ten questions that represent the three major conceptual domains of intake (questions one to three), dependence (questions four to six), and problems (questions seven to ten). Each question will receive a score from zero to four. For the last two questions: "No" scores zero, "Yes, but not in the last year" scores two, and "Yes, during the last year" scores four. The scores for each question are then totalled, with a score over eight indicating risky drinking patterns. For more detailed administration and scoring information, refer to the World Health Organization Guidelines ${ }^{[16]}$. The AUDIT has demonstrated validity among adolescents, drug-dependent clients, cross-cultural groups, drink drivers, emergency ward patients, and psychiatric patients. The AUDIT performed as well as the Michigan Alcoholism Screening Test (MAST) and the CAGE for identifying dependent drinking, and had higher sensitivity and specificity 
for detecting risky, non-dependent drinking. ${ }^{[17]}$ The AUDIT is also considered desirable because it is relatively short, easy to administer, and needs no formal training for administration.

A shortened version of the AUDIT, the AUDIT-C which consists of the alcohol consumption questions one to three, has been used successfully with male Veterans' Affairs patients to screen for heavy drinking. The AUDIT-C performed similarly to the full AUDIT for detecting heavy drinking. Patients were considered to be heavy drinkers if they drank more than 14 drinks a week or five or more drinks on one occasion in the past or a typical month. ${ }^{[18]}$

The prototype alcohol dependence questionnaire is the MAST. ${ }^{[19]}$ Instruments such as the MAST and the CAGE questionnaire were derived on the basis of their ability to distinguish chronic alcohol dependent individuals from non-alcohol dependent individuals. ${ }^{[20}$ Their performance is good in that 95 percent or more of chronic alcoholic dependent people are detected. These questionnaires are, however, much less satisfactory in detecting people with less severe drinking problems. Because of this limitation they are not advocated for screening in primary care settings. For screening in primary care settings, the AUDT is more appropriate than the MAST
or the CAGE for detecting less severe drinking problems.

\section{SCREENING FOR ALCOHOL-RELATED PROBLEMS}

There are a number of other screening instruments that measure alcohol-related problems. These instruments were developed to overcome the limitations of existing inventories, including the heavy emphasis on dependence questions, and the use of quantity and frequency measures that do not always detect the experience of alcohol-related problems.

The Alcohol Problems Questionnaire is a reliable 44-item instrument covering eight problem domains: friends, money, police, physical, affective, marital, children, and work. All questions relate to the past six months. Each of these domains yields a subscale score, and their combination provides a total or common score. Every positive response is scored as one. ${ }^{[2,22 !}$ Validated cut-off scores are not available.

The Alcohol-Related Problems Screening Questionnaire was developed in Australia. ${ }^{[23]}$ It is brief and has been used in both general practice and hospital settings. Another instrument that has been developed in Australia and subjected to validation studies is the Newcastle Alcohol-related Problems Scale (NAPS.) ${ }^{[24}$ Both of these scales are designed to assist in the efficient identification of individuals with alcohol-related problems associated with risky consumption, and thus their focus is broader than simply consumption or signs and symptoms of dependence. This gives them an advantage for use in screening activities. Moreover, the NAPS has the advantage of detecting problems arising from either the respondent's or from someone else's drinking, potentially allowing a greater rate of detection than is the case with other scales.

These scales will not detect all cases where risky consumption is present. For example, in the case of episodic intoxication it is less likely that the alcohol consumption will be detected as problematic. 


\section{Screening instruments for pregnant women: theT-ACE and theTWEAK}

Because of concerns that standard screening instruments may be less sensitive with women, or when used in prenatal clinics ${ }^{255}$, two screening instruments were developed for use with pregnant women. The T-ACE consists of three CAGE questions and a tolerance question. The TWEAK is a modified, five-item version of the MAST:

\section{T-ACE}

T Tolerance: how many drinks does it take to make you feel high?

A Have people Annoyed you by criticizing your drinking?

C Have you ever felt you ought to Cut down on your drinking?

E Eye opener: Have you ever had a drink first thing in the morning to steady your nerves or get rid of a hangover?

Source: Russell et al. 1994 [26]

\section{TWEAK}

$T$ Tolerance: how many drinks can you hold?

W Have close friends or relatives Womied or complained about your drinking in the past year?

E Eye Opener: do you sometimes take a drink in the morning when you get up?

A Amnesia: Has a friend or family member ever told you about things you said or did while you were drinking that you could not remember?

$\mathbb{K}(\mathrm{C})$ Do you sometimes feel the need to Cut down on your drinking?

Both the T-ACE and the TWEAK are more specific and sensitive in screening for risky drinking during pregnancy than either the MAST or the CAGE. ${ }^{[26]}$ The T-ACE is quick and easy to administer. A score of two or more indicates that the client may be drinking at risky levels, and that further investigation is required. The TWEAK has five items, with a score of two or more suggesting that the client is drinking at risky levels. 
Table 3.1: Alcohol use disorders identification test screening instrument

AUDIT: PLEASE CIRCLETHE ANSWERTHAT IS CORRECT FORYOU.

1. HOW OFTEN DO YOU HAVE A DRINK CONTAINING ALCOHOL?

Never Monthly or less 2-4 times a month 2-3 times a week 4 or more times a week
(0)
(1)
(2)
(3)
(4)

2. HOW MANY DRINIKS CONTAINING ALCOHOL DOYOU HAVE ON ATYPICAL DAY WHEN YOU ARE DRINIKING?

$\begin{array}{ccccc}1 \text { or } 2 & 3 \text { or } 4 & 5 \text { or } 6 & 7 \text { to } 9 & 10 \text { or more } \\ (0) & (1) & (2) & (3) & (4)\end{array}$

3. HOW OFTEN DOYOU HAVE SIX OR MORE DRINIKS ON ONE OCCASION?

Never Less than monthly Monthly Weekly Daily or almost daily
(0)
(1)
(2)
(3)
(4)

4. HOW OFTEN DURING THE LASTYEAR HAVE YOU FOUNDTHATYOU WERE NOT ABLETO STOP DRINIING ONCEYOU HAD STARTED?

\section{Never}
Less than monthly
Monthly
Weekly
Daily or almost daily

(0)
(1)
(2)
(3)
(4)

5. HOW OFTEN DURING THE LASTYEAR HAVEYOU FAILEDTO DO WHATWAS NORMALLY EXPECTED FROM YOU BECAUSE OF DRINIKING?
Never
Less than monthly
Weekly
Daily or almost daily

(1)

(2)

(3)

(0)

(2)

(4)

6. HOW OFTEN DURING THE LASTYEAR HAVE YOU NEEDED A FIRST DRINK IN THE MORNING TO GETYOURSELF GOING AFTER A HEAVY DRINIKING SESSION?
Never
Less than monthly
Monthly
Weekly
Daily or almost daily

(1)

(3)

(0)

(2)

(4)

7. HOW OFTEN DURING THE LASTYEAR HAVE YOU HAD A FEELING OF GUILT OR REMORSE AFTER DRINIKING?
Never
Less than monthly
Monthly
Weekly
Daily or almost daily

(0)

(1)

(2)

(3)

(4)

8. HOW OFTEN DURING THE LASTYEAR HAVEYOU BEEN UNABLETO REMEMBER WHAT HAPPENEDTHE NIGHT BEFORE BECAUSEYOU HAD BEEN DRINIKING?
Never
Less than monthly
Monthly
Weekly
Daily or almost daily

(0)

(1)

(2)

(3)

(4)

9. HAVEYOU OR SOMEONE ELSE BEEN INJ URED AS A RESULT OFYOUR DRINIKING? No
Yes, but not in the last year
Yes, during the last year

(0)

(1)

(2)

10. HAS A RELATIVE OR FRIEND OR A DOCTOR OR OTHER HEALTHWORKER, BEEN CONCERNED ABOUTYOUR DRINIKING OR SUGGESTEDYOU CUT DOWIN?
No
Yes, but not in the last year
Yes, during the last year

(0)

(1)

(2)

Source: World Health Organization, 1992 [27] 


\section{DETECTION USING MEDICAL HISTORY AND CLINICAL INDICATORS}

Certain physical disorders or signs are indicative of risky alcohol use. Common physical indicators include hypertension, a pattern of accidents, dilated facial capillaries, blood shot eyes, hand or tongue tremor, history of gastrointestinal disorders, duodenal ulcers and cognitive deficits. ${ }^{[2,29]}$ Conditions such as liver cirrhosis and pancreatitis are commonly alcohol-induced. Subtler signs include work, financial, marital and relationship problems, domestic violence, insomnia, depression and anxiety. ${ }^{30]}$

While the above problems are indicative of alcohol misuse, they are not conclusive. Nor does their absence rule out the existence of risky alcohol consumption.

\section{DETECTION USING BIOLOGICAL MARKERS}

A number of biological markers are used to detect alcohol consumption: serum GGT, aspartate (AST), alanine aminotransferase (ALT), HDL-cholesterol, uric acid, mean cell volume, and carbohydrate-deficient transferin (CDT). Serum GGT, a liver enzyme, is elevated in 60 to 80 percent of alcoholics. Newer markers, including CDT and antibodies to acetaldehyde-protein abducts, offer fair prospects for early detection. A CDT test kit is now available and is in use at some clinics in Australia. GGT is also used in some clinical settings.

The other generally available laboratory tests are less sensitive: for example, an elevated mean cell volume is found in only 5 to 20 percent of alcoholic patients. The value of these tests in detecting non-alcohol dependent people with risky alcohol consumption is correspondingly lower.

The combination of a number of biological markers can provide a rate of detection above the rate achievable by any biochemical marker alone, with a sensitivity of 78 percent. ${ }^{[31]}$ However, combinations of tests are not recommended for clinical use because of reduced specificity. ${ }^{[32]}$

\footnotetext{
D Because of the greater sensitivity and specificity of questionnaire approaches (for example, the AUDT) these are preferred to biological markers. Biological markers should only be used as an adjunct to other screening measures.
} 


\section{AND RETROSPECTIVE DIARY}

The retrospective diary $(\mathrm{RD})$ requires drinkers to identify the type and quantity of alcoholic beverage consumed beginning with the previous day and working back through each day of the week. ${ }^{\text {[3] }}$ The Quantity-Frequency Index (QFI) question asks the drinker to indicate the number of occasions during the previous 30 days on which they had consumed four different levels of standard drinks as defined by NHMRC guidelines ${ }^{[34}$ :

\section{TABLE 3.2: LEVELS OF STANDARD DRINKS}

\begin{tabular}{lcc}
\hline Levels of standard drinks & Males & Females \\
\hline Level 1 & 7 to 10 & 5 to 8 \\
Level 2 & 11 to 15 & 9 to 13 \\
Level 3 & 16 to 20 & 14 to 18 \\
Level 4 & $>20$ & $>18$ \\
\hline
\end{tabular}

The lowest categories ( 7 to 10 drinks for males and 5 to 8 drinks for females) were used as the cut-off score, that is any respondents who identified an occasion of drinking above those levels were classified as binge drinkers (Table 3.2).

Although the $\mathrm{RD}$ takes longer to administer than the QFI (mean completion times of three minute, 38 seconds and one minute, 41 seconds respectively), it provides two important pieces of information: weekly and binge consumption. Further, while the $\mathrm{RD}$ was inferior in detecting binge drinking, the $\mathrm{QFI}$ underestimated overall drinking relative to the $\mathrm{RD}$. ${ }^{[35]}$ 
In routine general practice, without specific screening techniques, 75 percent of risky/high risk drinkers are not detected.

There is strong evidence that health care providers in general practice and hospital settings can effectively screen for and intervene with risky/high risk drinkers. Screening can also be usefully conducted in community health care settings and workplaces.

The AUDIT is a reliable, valid way of screening for risky drinking in primary care settings: it has demonstrated validity among adolescents, drug-dependent clients, a range of cross-cultural groups, drink drivers, emergency ward patients, and psychiatric patients.

The T-ACE and the TWEAK are more specific and sensitive in screening for risky drinking in pregnant women than the MAST or the CAGE.

The Alcohol Problems Questionnaire (APQ) is a valid and reliable measure of alcohol-related problems, and is relatively independent of levels of consumption.

The Quantity-Frequency Index and the retrospective diary are both reliable ways of identifying risky/high risk levels and patterns of consumption.

Screening for risky and high risk alcohol consumption

should be widely implemented in general practice, hospitals, community health care, and workplaces.

The AUDIT, the Quantity-Frequency Index or the retrospective diary are recommended for screening in the above settings.

The T-ACE and the TWEAK are recommended for screening risky drinking in pregnant women. 


\section{In-depth assessment before more intensive treatment}

\section{SECTION AIM}

The aim of this section is to guide assessment which might take place before a relatively intensive and extensive intervention for those drinkers who:

- Have not responded to brief advice to cut down their drinking

- Have severe alcohol related problems

- Have asked for specialised assistance

- Are clearly in need of specialist help in dealing with their drinking

This level of assessment is not appropriate for brief intervention.

In the preceding section, guidelines are provided for brief, routine screening for proactive interventions. Assessment intensity and detail varies across settings, the amount of assessment being related to the level of specialisation in alcohol problems.

This section covers several areas for assessment:

- motivation to change

- alcohol dependence and consumption pattern

- concurrent psychological and psychiatric problems

- family factors

- physical well-being and cognitive functioning

From the first contact with the client there is a need to instil in the client a sense of hope and a belief that change is possible. This is especially important in clients who have tried to alter their drinking and failed. Self-efficacy, that is the client's belief that there is something they can do about their problem, is an important factor in treatment success. ${ }^{360}$ Self-efficacy may in turn be influenced by the therapeutic relationship. ${ }^{[37}$ (see Chapter 8: Psychosocial interventions)

\section{The need for comprehensive assessment must be ballanced with engaging and retaining the client.}

If the client perceives that little or no progress is being made in the first sessions, their motivation to stay in treatment may reduce, and they will leave. The assessment process might be spread over several sessions, allowing some time in each session for setting preliminary treatment goals and working towards those goals. As more in-depth assessment occurs, these treatment goals and strategies may need to be adjusted. 


\section{THE PURPOSE OF ASSESSMENT}

Before intensive and extensive interventions, assessment needs to be carefully conducted. Assessment has two important functions:

1. It assists the client and clinician to develop shared treatment goals and a treatment plan. Different patients will require different approaches, as risky drinkers and alcohol dependent people do not have a homogeneous group of problems. Any underlying and accompanying problems must be identified and treated, even if the causal relationship is not clear.

2. It provides an opportunity for clinician and client to develop a rapport. If the clinician shows the client empathy and courtesy and provides a sense of hope and optimism, the client is less likely to take a defensive stance in the interview, and is more likely to look at the opportunity as one that facilitates change. In this shared process, feedback from the clinician can encourage the client to appraise their situation from a new perspective. Assessment could be defined as the beginning of therapy, and sympathetic understanding of the implications of this for the drinker and their family is important. In particular, it is important to highlight the client's perception of the opportunity for change, and this requires the clinician to have a positive and realistic approach.

\section{KEY ISSUES IN ASSESSMENT:}

- Assessment is one of the earliest opportunities the clinician has for engaging and retaining the client in treatment.

- Intensive treatment for alcohol problems should begin with a comprehensive assessment so that the most appropriate intervention(s) can be selected.

- Assessment should be balanced with achieving treatment progress so that the client remains motivated.

- Assessment should lead to agreed treatment goals and a treatment plan. The treatment plan should be based on the most effective intervention for the client, not just on the kind of treatment typically provided by the agency. It is preferable that the client be informed about the range of options for intervention available locally and assisted to make a reasoned decision as to which intervention is most suited to his or her needs. In this way, assessment should help to guide client-treatment matching.

- Assessment should continue throughout treatment as the client's progress is measured against the treatment goals.

- Assessment should combine a variety of techniques for gathering information about the client, including diagnostic interviews, standardised questionnaires, medical examinations, and/or biological markers.

- Much information can be best collected in a semi-structured, open-ended interview, using a guided exploration of the client's subjective experience of drinking. This has the advantage of clinician involvement which is personal and responsive to the drinker, rather than mechanical and impersonal. Yet, it should maintain a purposeful structure so as to avoid a vague, directionless discussion of the drinker's history.

- The assessment should emphasise the client's present situation. Information about past experiences is useful in clarifying how the client came to be in the present situation and what is maintaining maladaptive thoughts and behaviours. However, the assessment should be geared to collecting information that will help to tailor treatment so that it is appropriate for the client. 


\section{ENGAGINGTHE CLIENT INTREATMENT}

Client engagement may be viewed in terms of intensity and duration of treatment participation. Higher levels of engagement are predictive of positive treatment outcomes and are, in tum, contingent upon both client characteristics - for example pre-treatment motivation, higher pre-treatment alcohol consumption, more pre-treatment arrests, higher levels of concentration - and treatment experiences: strength of the therapeutic relationship, perceived helpfulness of the treatment services, empathy of the clinician, removal of practical barriers such as transportation, and the inclusion of relapse prevention training. ${ }^{[38,39]}$ Clinician characteristics and the therapeutic relationship are also crucial to engaging the client in treatment (see Chapter 8: Psychological interventions for more information on this issue). ${ }^{37]}$

\section{Goal-setting and treatment planning}

The client's perception of a gap between their goals and their present state may improve motivation for change. ${ }^{\left[{ }^{[6]}\right]}$ Acknowledging the client's goals rather than insisting on a particular set of goals is more effective motivationally. ${ }^{\text {[40 }}$ Sanchez-Craig claims that offering clients choices about treatment goals and strategies produces better outcomes. ${ }^{[41]}$ There is also evidence that providing the client with a choice of treatment options improves treatment retention. ${ }^{[42]}$

\section{THE INITIAL INTERVIEW}

The assessment procedure ideally takes the form of a semi-structured interview where the client and the clinician compile a narrative history, using questionnaires as appropriate and necessary. The clinician should cover the important areas for assessment presented in Table 3.3. Specific areas for assessment are covered in detail in the following sections. It is recommended that these areas be covered in two or more sessions. Assessment is an ongoing part of the treatment process, and it should not be viewed as something that occurs in isolation, to be ignored later.

In some settings, for example psychiatric or medico-legal settings, a more structured interview may be appropriate. The Composite International Diagnostic Interview (CIDI) is a standardised and comprehensive interview designed to assess psychological disorders against the International Classification of Diseases (ICD) and Diagnostic Statistics Manual-IV (DSM-IV) diagnoses. It must be administered or supervised by a fully trained mental health professional who has undertaken recognised CIDI training. As well as substance use disorders, it covers eating disorders, organic mental disorders, schizophrenic disorders, paranoid disorders, affective disorders, anxiety disorders, somatisation disorders, dissociative disorders, and psychosexual disorders.

The CIDI, the Schedules for Clinical Assessment in Neuropsychiatry (SCAN) and the Alcohol Use Disorder and Associated Disabilities Interview Schedule-Alcohol/Drug-Revised (AUDADIS-ADR) all have reasonable test-retest reliability and diagnostic concordance for alcohol dependence, but not for risky alcohol use or abuse.

The sections that follow cover specific areas that may require assessment. Each of these areas needs to be covered to ensure a comprehensive assessment. It is not necessary for every client to be assessed extensively on each of the areas. In some cases, such a detailed assessment is unnecessary as the status of the client will be obvious. In other cases the information provided below allows the clinician to carry out a careful assessment of the important aspects. 


\section{TABLE 3.3: ISSUESTO BE COVERED INTHE INITIAL CLINICAL INTERVIEW}

\section{INITIAL ASSESSMENT}

- Presenting problems

- Role of drinking/drug use in presenting problems

- Motivation for change

- Other concerns, for example relationship issues, work problems, legal problems

\section{DRINKING/DRUG USE ASSESSMIENT}

- Quantity, frequency, pattern of drinking

- Last drink/drug use

- Length of drinking/drug problem

- Consequences of drinking/drug use

- DSM-IV symptoms of abuse or dependence

- Assessment of need for detoxification

\section{ASSESSMENT OF OTHER PROBLEMS}

- Psychotic symptoms

- Depression

- Anxiety

- Cognitive impairment

- Physical wellbeing

- Family issues 


\section{ASSESSING MOTIVATION}

Motivation to change is an important mediating variable in treatment outcome, so enhancing the drinker's level of motivation may be an important aspect of treatment. ${ }^{[43]}$ Perhaps the simplest method for assessing a drinker's readiness to change is through direct questioning during the assessment interview. This should only be done after risky alcohol consumption has been discussed, and the patient has received feedback on their level of drinking. Two questions that might prove useful are:

1 .How interested are you in changing your drinking now?' and

2. "Do you feel that you ought to stop drinking, or do you really want to?".

The client may also be asked:

1 'What would you be prepared to do to solve this drinking problem?'

2. "How confident are you that you can achieve this?"

3. "Are you prepared to attend the next appointment?"

The client may be encouraged to explore the various treatment options from the perspective of motivation to participate. Altematively, as developed elsewhere [44] the client may simply be asked:

"How do you feel about your drinking at the moment?"

Responses may vary from:

1 .I'm happy with my drinking", "I enjoy drinking", "I'm not interested in stopping drinking"; through to

2. contemplative responses such as "I'm thinking about stopping", "I'm not sure if I'm ready at the moment", "I'm interested in weighing up stopping"; to

3. action-oriented responses such as "I want to stop now", "I may need some help", or "The disadvantages of drinking outweigh the benefits for me".

\section{These questions should be asked with curiosity and a willingness to explore the client's answers, not in a confronting or adversarial way.}

The client's responses may indicate a need for a motivational intervention, particularly for the ambivalent drinker, methods for which are outlined in Chapter 8: Motivational interviewing.

The stages of change model, also known as the transtheoretical model (TTM), includes change processes and levels of change. ${ }^{[45]}$ However, assessment tools are primarily available to measure stages of change; thus our discussion is limited to this aspect of the model. Although the model is widely used there is equivocal evidence of its ability to predict treatment outcome with alcohol dependent patients. ${ }^{[46,4]}$ The model though, appears to identify some differences between drinkers at various stages. ${ }^{[48]} \mathrm{A}$ number of reviewers have concluded that the stages are not mutually exclusive; there is little evidence of sequential movement through discrete stages; and better quantitative outcome studies are needed. ${ }^{49,50,51]}$ One problem may be that the link between stages and particular treatments has been oversimplified. ${ }^{[52]}$ For example, people in the preparation and action stages may still be confused over the costs and benefits of changing their drinking behaviour, and continue to have doubts about making such changes. 
Because of the mixed evidence supporting the model, we recommend that the stages of change model be used with caution and that motivational interviewing strategies be used whenever clients appear to be struggling with decisions, not just at the beginning of treatment.

Two questionnaires have been designed to assess the drinker's readiness to change: the University of Rhode Island Change Assessment (URICA) scale, and the Readiness to Change Questionnaire (RTCQ). URICA is a 32-item questionnaire with subscales corresponding to the stages of change. URICA seems to produce consistent profiles corresponding to the stages of change, which in turn predicts alcohol and drug use severity. ${ }^{[3]}$

The RTCQ is a 12-item questionnaire with three subscales that correspond to the pre-contemplation, contemplation and action stages of the model. Although the RTCQ demonstrated reasonable predictive validity with a sample of male risky drinkers in a general hospital setting ${ }^{[54]}$, its usefulness in clinical settings is yet to be determined, given the low internal reliability of the pre-contemplation and contemplation subscales. ${ }^{[55]}$

\section{ASSESSINGTHE CONSEQUENCES OF DRINKING}

It is important for the clinician to assess the range of problems encountered by the client as a result of their drinking. In addition to health and family problems, the client's drinking may have detrimentally affected work performance, social relations or financial stability. Alcohol-related offences such as drink-driving might also be relevant. A specific crisis about one of these factors may have been the impetus for seeking help, and this should be explored. Discussion of the "less good things" about drinking can also help to enhance the client's readiness for change (see the motivational interviewing material in Chapter 8: Psychosocial interventions).

The Alcohol Problems Questionnaire (APQ) is a reliable instrument that covers eight domains:

- friends

- money

- police

- physical

- affective

- marital

- children

- work

For more information on the APQ, see the Screening section earlier in this chapter. 


\section{ASSESSING LEVEL AND HISTORY OF ALCOHOL CONSUMPTION}

This part of the assessment process should gather information about the drinking history, including how the risky drinking pattern evolved, fluctuated and/or progressed. The assessment should include the client's reconstruction of a typical drinking day and week, from the time of waking through all the day's activities. This information can be used when planning relapse prevention procedures with the client.

Edwards (1982) has recommended that clients be asked to timetable their drinking on a typical day. ${ }^{[5]}$ For example, the clinician might ask at what time the first drink is taken, where and with whom. The time spent drinking or the money spent on alcohol can be compared with the client's estimate of the amount of alcohol consumed to test the accuracy of that estimation. Consumption can be linked to particular events, behaviours and times. An assessment of a typical day also gives information about the antecedents and consequences of drinking.

Careful probing in the interview assists the client to make accurate estimations of the amount of alcohol usually consumed. It is useful for the clinician to suggest a level of drinking that is higher than expected so that the client is most likely to feel comfortable in admitting the real level of drinking by bringing the estimation down to the correct level.

\section{Quantity of alcohol consumed is not the only way to look at alcohol consumption. The clinician also needs to assess frequency of drinking, lt is strongly recommended that the assessment also look at the client's pattem of drinking.}

The clinician needs to distinguish between daily drinking and binge drinking where the weekly or monthly consumption is concentrated over several days and the client is abstinent or drinks lightly at other times. There are many methods available for this assessment, ranging from diary methods, using a calendar to assess drinking over the previous month or months, as well as gathering information from a spouse or other person close to the drinker to validate the drinker's report.

The Timeline Follow back Method (TLFB) helps to obtain an accurate, retrospective account of alcohol consumption over a particular period, typically three months. ${ }^{[57]}$ This method requires the client and clinician to fill in a blank calendar with a detailed description of alcohol consumption. The client is first asked to note all events that may assist with recall, for example public holidays or significant personal events. Any personal diaries may help with recall. The client then fills in the drinking days, noting the amount consumed, and perhaps also the number of hours of consumption.

The Brief Drinker Profile and the Comprehensive Drinker Profile allow for a standardised assessment of both "steady pattern" drinking and binge drinking. ${ }^{[5,59]}$ Other drug use, including use of sedative medications and illicit drugs, should also be assessed. 


\section{ASSESSING ALCOHOL DEPENDENCE}

The measurement of the degree to which a drinker is dependent upon alcohol allows the clinician to plan the treatment goals. A person who is less alcohol dependent may be able to achieve controlled drinking. The dependence level indicates whether the client can expect to experience withdrawal and might also provide some initial indication of how intense the treatment program needs to be.

The DSM-IV-R criteria for the alcohol dependence and abuse syndromes are presented in Appendix three. ${ }^{[60]}$ There are several questionnaires that measure alcohol dependence and two of these are included in these guidelines.

The SADQ-C has three sections and is shown in Table $3.4{ }^{\left[{ }^{[61]}\right.}$. The Short Alcohol Dependence Data questionnaire (SADD) ${ }^{[62]}$ is shown in Table 3.5.

\section{The SADQ-C}

Answers to each question are rated on a four-point scale as follows:

$0=$ almost never

$1=$ sometimes

$2=$ often

$3=$ nearly always

\section{Section B and Section C SADQ-C scores lower than or equal to 30 indicate low (zero to 20) to moderate dependence, while scores higher than 30 indicate a high level of dependence.}

This dichotomy has been used in the past as a point at which drinkers who exceed the cut-off should be advised that abstinence is the appropriate goal, as well as being used to determine kind and intensity of intervention. The SADQ-C emphasises tolerance and withdrawal symptoms, and physical dependence generally. ${ }^{[63]}$ The impaired control items are a new inclusion, but they do not feature in the current scoring of dependence, and should not be used in reaching an overall score on the SADQ-C. The impaired control items form a separate scale. ${ }^{[1]]}$

\section{The SADD}

By way of contrast, the SADD questionnaire measures physiological and behavioural features of dependence, such as the salience of the drink-seeking behaviour. ${ }^{[2]}$ Its authors have recommended that scores of one to nine be considered low dependence, 10 to 19 medium dependence, and 20 or more high dependence, on the basis of a four-point rating scale similar to that used in the SADQC. Other questionnaires are available to the interested reader, including the Alcohol Dependence Scale (ADS). ${ }^{[64]}$ 
The client can complete these questionnaires during assessment or, altematively, they can provide a checklist to help organise the clinician's questions. Explore the client's experiences of dependence and tolerance by asking the client to describe the last two or three occasions on which they reached intoxication and the last two or three occasions when they did not become intoxicated.

No test or measure should be used in an absolute or rigid way to determine the kind of intervention that might be appropriate. Moreover, it should be recognised that dependence is used, to an extent, as a proxy for the severity of the alcohol problems and although it is a useful concept, there is a need to assess the severity of the person's alcohol related problems more widely. The implication that more severely dependent and affected drinkers require a more intense intervention is relevant for three aspects of treatment delivery:

- These people may require more attention and more time during treatment.

- Treatment might be spread over a longer period and/or come from multiple sources.

- Treatment might be more strategically focussed on the particular problem and deficits that are apparently responsible for the risky drinking. 


\section{TABLE 3.4: SEVERITY OF ALCOHOL DEPENDENCE QUESTIONNAIRE FORM-C (SADQ-C)}

\section{SADQ-C}

Name: ................ Sex: M/ F Date of birth:

.Age: . . . . . . .

Have you drunk any alcohol in the past six months? YES / NO.

If YES, please answer all the following questions by circling the most appropriate response.

\section{(Section A - ICQ) During the past SIX MONTHS:}

1. AFTER HAVING JUST ONE TO TWO DRINKS, I FELT LIKE HAVING A FEW MORE.

Never or almost never Sometimes Often Nearly always

2. After having two or three drinks, I could stop drinking if I had other things to do.
Never or almost never
Sometimes
Often
Nearly always

3. When I started drinking alcohol, I found it hard to stop until I was fairly drunk.
Never or almost never
Sometimes
Often
Nearly always

4. When I went drinking, I planned to have at least six drinks.

Never or almost never Sometimes Often Nearly always

5. When I went drinking, I planned to have no more than two or three drinks.

Never or almost never Sometimes Often Nearly always

\section{(Section B - SADQ, Form-C) During the past SIX MONTHS:}

1. The day after drinking alcohol, I woke up feeling sweaty.
Never or almost never
Sometimes
Often
Nearly always

2. The day after drinking alcohol, my hands shook first thing in the morning.
Never or almost never
Sometimes
Often
Nearly always

3. The day after drinking alcohol, I woke up absolutely drenched in sweat.
Never or almost never
Sometimes
Often
Nearly always

4. The day after drinking alcohol, my whole body shook violently first thing in the morning if I didn't have a drink.
Never or almost never
Sometimes
Often
Nearly always

5. The day after drinking alcohol, I dread waking up in the morning.
Never or almost never
Sometimes
Often
Nearly always

6. The day after drinking alcohol, I was frightened of meeting people first thing in the morning.
Never or almost never
Sometimes
Often
Nearly always

7. The day after drinking alcohol, I felt at the edge of despair when I awoke.

Never or almost never

Sometimes

Often

Nearly always

(Section B - SADQ, Form-C) During the past SIX MONTHS: cont. 
8. The day after drinking alcohol, I felt very frightened when I awoke.
Never or almost never
Sometimes
Often
Nearly always

9. The day after drinking alcohol, I liked to have a morning drink.
Never or almost never
Sometimes
Often
Nearly always

10. The day after drinking alcohol, in the morning I always gulped my first few alcoholic drinks down as quickly as possible.

Never or almost never Sometimes Often Nearly always

11. The day after drinking alcohol, I drank more alcohol in the morning to get rid of the shakes.
Never or almost never
Sometimes
Often
Nearly always

12. The day after drinking alcohol, I had a very strong craving for an alcoholic drink when I woke. Never or almost never Sometimes Often Nearly always

13. I drank more than a quarter of a bottle of spirits in a day ( or 1 bottle of wine or 7 middies of beer).

Never or almost never Sometimes Often Nearly always

14. I drank more than half a bottle of spirits in a day ( or 2 bottles of wine or 15 middies of beer). Never or almost never Sometimes $\quad$ Often Nearly always

15. I drank more than one bottle of spirits per day ( or 4 bottles of wine or 30 middies of beer). $\begin{array}{llll}\text { Never or almost never } & \text { Sometimes } & \text { Often } & \text { Nearly always }\end{array}$

16. I drank more than two bottles of spirits per day ( or 8 bottles of wine or 60 middies of beer). $\begin{array}{llll}\text { Never or almost never } & \text { Sometimes } & \text { Often } & \text { Nearly always }\end{array}$

\section{(Section C - SADQ, Form-C) Imagine the following situation:}

(1) You have HARDLY DRUNK ANY ALCOHOL FOR A FEW DAYS.

(2) You then drink VERY HEAVILY for TWO DAYS.

How would you feel the MORNING AFTER those two days of heavy drinking?

17. I would start to sweat

Notatall Slightly Moderately Quite a lot

18. My hands would shake

Notat all Slightly Moderately Quite a lot

19. My body would shake

Not at all

Slightly Moderately Quite a lot

20. I would be craving for a drink

Not at all Slightly Moderately Quite a lot

Source: Stockwell et al. (1994) [61] 


\section{TABLE 3.5:THE SEVERITY OF ALCOHOL DEPENDENCE DATA (SADD) QUESTIONNAIRE}

SADD: The following questions cover a wide range of topics to do with drinking. Please read each question carefully but do not think too much about its exact meaning. Think about your MOST RECENT drinking habits and answer each question by placing a tick ( .) under the MOST APPROPRIATE heading. If you have any difficulties ASK FOR HELP.

Never Some- Often Nearly

times always

1. Do you find difficulty in getting the thought of drink out of your mind?

2. Is getting drunk more important than your next meal?

3. Do you plan your day around when and where you can drink?

4. Do you drink in the morning, afternoon and evening?

5. Do you drink for the effect of alcohol without caring what the drink is?

6. Do you drink as much as you want irrespective of what you are doing the next day?

7. Given that many problems might be caused by alcohol do you still drink too much?

8. Do you know that you won't be able to stop drinking once you start?

9. Do you try to control your drinking by giving it up completely for days or weeks at a time?

10. The morning after a heavy drinking session do you need your first drink to get yourself going?

11. The morning after a heavy drinking session do you wake up with a definite shakiness of your hands?

12. After a heavy drinking session do you wake up and retch or vomit?

13. The morning after a heavy drinking session do you go out of your way to avoid people?

14. After a heavy drinking session do you see frightening things that later you realise were imaginary?

15. Do you go drinking and the next day find you have forgotten what happened the night before?

Source: Raistrick, et al. $1983^{[62]}$ 
The level of dependence can assist in determining the appropriate goal for the drinker: moderation or abstinence. Heather (1989) has used clinical judgement and research experience to develop a set of suggested ranges on these instruments for determining both the goal and the intensity of the intervention that might be used. ${ }^{[65]}$ His scheme is partly produced in Table 3.6. Heather notes that the range of scores is not based on empirical data, and that there is no evidence to suggest that the ranges are equivalent. They should be treated as "rule of thumb". He also indicates that it "cannot be stated too strongly that allocation to moderate drinking or abstinence goals and to brief and intensive interventions are essentially clinical decisions, depending critically on the unique circumstances of the individual client". ${ }^{[65]}$

A SADQ score should not be the sole criterion for determining the intensity of treatment because the cut-off point might exclude some people who are clearly dependent upon alcohol in a psychological sense, or who require a more intense intervention than their dependence level might suggest was necessary. Therefore all aspects of the drinker's presentation should be considered when assisting him or her to choose an appropriate treatment goal and strategy.

\section{TABLE 3.6: SUGGESTED SCORES ONTHREE MEASURES OF ALCOHOL DEPENDENCETO} DETERM INETREATMENT GOAL AND INTENSITY

$\begin{array}{lccc}\text { Scale } & \begin{array}{c}\text { Low dependence; } \\ \text { Moderation goal; } \\ \text { Brief intervention }\end{array} & \begin{array}{c}\text { Moderate dependence; } \\ \text { Moderation/abstinence; } \\ \text { Brief or intensive intervention }\end{array} & \begin{array}{c}\text { High dependence; } \\ \text { Abstinence goal; } \\ \text { Intensive intervention }\end{array} \\ \text { SADQ } & 0-20 & 21-40 & 41-60 \\ \text { SADD } & 0-9 & 10-19 & 20-45 \\ \text { ADS } & 0-13 & 14-30 & 31-51\end{array}$

Source: Heather (1989) ${ }^{[65]}$

\section{ASSESSING PSYCHOLOGICAL PROBLEMS AND PSYCHIATRIC DISORDERS}

Assessment of the presence of psychiatric comorbidity and psychological problems among alcohol dependent patients is essential, and when problems are detected, ensuring that the patient receives an appropriate intervention must be a high priority. Psychological problems and psychiatric comorbidity are more prevalent among people who are alcohol dependent than in the general population. ${ }^{[6]]}$

\section{Depressive and anxiety disorders are the most prevalent psychiatric disorders among alcohol dependent persons.}

The specialist interventions required to resolve serious psychiatric disorders may not be within the range of qualification or experience of most drug and alcohol workers, be they nurses, doctors, or social workers/psychologists. It is important that:

- All drug and alcohol counsellors and professional personnel in this area develop links with other services that can deal with these disorders.

- The presence of behavioural or affective problems be assessed in the narrative interview with questions about mood, fears and anxieties. Alternatively, there are a number of symptom 
checklists that can be used to assess for the more common disorders. These include the Beck Depression Inventory ${ }^{[67]}$, the Beck Anxiety Inventory ${ }^{[6]}$, the Hopkins Symptom Checklist 90Revised ${ }^{[69]}$, and the Depression, Anxiety and Stress Scale (DASS, ${ }^{[00]}$ ). The responses to these questionnaires can form the basis for a discussion with the client about psychological problems. Where it is suspected that there is some psychological problem present, a full diagnostic assessment should be undertaken by a suitably qualified professional, that is, a psychiatrist or a clinical psychologist.

- Clients are reassessed after three or four weeks of treatment, and a final psychiatric diagnosis is delayed until this time: it is likely that many of these disorders are reactions to the chaos and disarray in the client's life that are associated with the risky drinking, or to the neurological effects of alcohol. Therefore some of these apparent disonders resolve without formal therapy when the drinking ceases or decreases. Depressive disorders can be expected to lessen in severity with ongoing abstinence in a substantial number of cases. Anxiety may also be caused by the drinking problem, rather than the reverse. However, serious anxiety disonders may be present and can lead to relapse.

- Questions about sexual abuse should be framed in a non-threatening way so that the client can discuss the experience without fear of rejection. There is evidence that a high percentage of alcohol-dependent women in treatment have had some experience of physical and sexual abuse. ${ }^{[1]}$ Women with a history of child sexual abuse who are pressured to discuss the issue with non-specialist counsellors in the context of treatment for drug and alcohol problems may have worse outcomes at six months compared to women who have not received this form of treatment. Based on these trends, and drawing on clinical expertise, it has been argued that if child sexual abuse is an issue, the client should be referred for a specialist intervention. Although caution should be exercised in addressing the issue, clinicians need to discuss the issue without seeming too tentative or fearful. In some jurisdictions, training in dealing with child sexual abuse is now available for alcohol and drug counsellors. A number of jurisdictions have set up services for treating victims of child sexual assault but resources are limited.

- Assessment covers subclinical symptoms of depression and anxiety. Some symptoms may not be obvious to the clinician inexperienced in the area. Because of the risk of missing such symptoms at the initial assessment it is important to ask the client directly whether he or she has had problems with relaxing or in dealing with stress. A positive response may indicate the need to offer an intervention to deal with these issues. Similarly, the presence of difficulties in interpersonal situations, again often subclinical and within "normal" limits, can result in some emotional turmoil which causes the person to drink. Again the clinician is advised to discuss these problems with the drinker. The presence of such problems may suggest the need to deal with interpersonal skills, such as assertiveness and other social skills. These and other such interventions are covered later in this chapter. 
- Suitable scales for the assessment of comorbid mental health disorders include:

$\triangleright \quad$ The General Health Questionnaire (GHQ) is designed as a screening instrument to identify likely non-psychotic psychiatric "cases" in general health settings. ${ }^{[72]}$

$\triangleright \quad$ The Short Form 12 (SF-12) assesses possible limitations in both physical and mental health. [73]

$\triangleright \quad$ The Beck Depression Inventory measures depression and its symptoms. ${ }^{[6]}$

$\triangleright \quad$ The Beck Hopelessness Scale measures hopelessness and negative views about the future, and an indicator of suicide attempts. ${ }^{[74]}$

$\triangleright \quad$ The Spielberger State-Trait Anxiety Scale measures current anxiety - state anxiety - and a more enduring personality characteristic - trait anxiety. ${ }^{[75]}$

$\triangleright \quad$ The Social Anxiety Interaction Scale and the Social Phobia Scale are useful for assessing social phobia. ${ }^{[76]}$

$\triangleright \quad$ The Modified PTSD Symptom Scale is a brief (17-item) measure of post-traumatic stress disorder symptoms. ${ }^{[7]}$

$\triangleright \quad$ The Acute Stress Disorder Scale is a measure of acute stress disorder symptoms. ${ }^{[7]}$

$\triangleright \quad$ The Eating Attitudes Test is used to detect disturbed eating patterns. ${ }^{[9]}$

$\triangleright \quad$ The Kessler 10 Symptom Scale is a scale of psychological distress, suitable for use as an outcome measure in people with anxiety and depressive disorders. It is likely to become the standard scale for use by general practitioners and mental health workers. ${ }^{180]}$

\section{ASSESSING COGNITIVE FUNCTIONING}

Formal assessment of alcohol related brain damage is a specialist activity that is outside the scope of most drug and alcohol workers, and referral may be necessary to a psychologist or a neuropsychologist. Nevertheless, since there is a high prevalence of cognitive dysfunction among people with alcohol problems ${ }^{\left[{ }^{[1]}\right.}$, drug and alcohol workers should screen for deficits, even if such an assessment is limited. Only some clients presenting for treatment for alcohol problems will need to be formally assessed for cognitive dysfunction, that is, those whose deficits interfere with dayto-day functioning and participating in treatment.

It is estimated that more than 50 percent of patients over the age of 45 who have lengthy histories of drinking at risky levels will show some degree of cognitive dysfunction, although this may not be permanent. ${ }^{[82]}$ In fact, between 75 percent and 100 percent of patients admitted to alcohol treatment facilities perform below normal for their age groups on tests of cognitive function. ${ }^{[83]}$ If the client has pronounced organic brain damage, the problems are usually obvious.

\section{For other clients who have alcohol-related cognitive dysfunction there might not be olbvious behavioural signs of impairment, and specific tests are required to detect and to measure the extent of the deficits.}

The drug and alcohol professional must be aware of the possibility of alcohol-related brain damage and be watchful for signs of it in the clinical interview. This section provides some information 
about the kinds of deficits that can occur and the range of methods available to detect them. See Chapter 11: Interventions for specific client groups for more information about treatment.

\section{Cognitive impairment associated with alcohol abuse and dependence}

Amnesia associated with Wernicke Korsakoff's syndrome (WKS) leaves short term memory and memory for some long term events intact, but particularly interferes with learning new information. A person suffering from the amnesic syndrome might, for example, not recognise their doctor even though she or he visited only yesterday. The client might also show deficits in planning, organisation, and problem-solving. The prevalence of Wernicke Korsakoff's syndrome has reduced in Australia since the introduction of thiamine enriched bread flour. However, there is evidence that some single symptoms of Wernicke Korsakoff's syndrome are present in about one-third of alcoholdependent people, and that lower estimates are because of the difficulty in diagnosing Wernicke Korsakoff's syndrome. ${ }^{[81]}$

Cognitive deficits such as impairment in verbal abilities, visual-spatial abilities, problem-solving skills and memory often improve with a period of abstinence from alcohol. ${ }^{[3] 1}$ However, they can still hamper the effectiveness of treatment programs. These deficits may impair motivation, attention span, the capacity to evaluate situations critically or the ability to acquire new skills. Therefore interventions during this early stage may need to be kept relatively simple until or unless it is apparent that the client can cope with more complex information. The possibility of improving cognitive functioning should be taken account of in the assessment process by allowing a sufficient period of abstinence from alcohol to elapse before finalising assessment of cognitive dysfunction.

\section{Assessment Instruments for Cognitive Impairment}

For the non-psychologist who is seeking a quick screening for cognitive dysfunction, the Mini-Mental State Examination is helpful. ${ }^{[84}$ The use of age-and education-specific cut-off scores may improve sensitivity without affecting specificity. Because the test may have limited sensitivity to subtle deficits, care should be taken not to interpret an apparent lack of cognitive deficit as a real lack of deficit. ${ }^{[5]}$ To ensure optimal performance, testing should not be conducted during detoxification and the client must be sober during testing. The clinician also needs to be aware of the effects of anxiety in the testing situation as extreme anxiety reduces performance. It is recommended that drug and alcohol workers be trained in basic screening for marked cognitive dysfunction, such as that represented in the Mini-Mental State Examination. ${ }^{[84]}$

For a more extensive assessment, a variety of tests have been shown to be sensitive to alcoholrelated brain damage. These tests include the Rey Complex Figure Test, designed to test perceptual organisation and visual memory, the Rey Auditory-Verbal Learning Test which measures verbal memory recall and recognition, and the Trail Making Test which tests visual concepts and visuomotor tracking. However, some of these tests have very limited normative data available and they are not specific to alcohol-related brain damage, so that their confident interpretation is made difficult. Also some of the tests relevant to the detection of cognitive dysfunction should only be administered by psychologists who have been trained in their interpretation. This is the case for the Wechsler Adult Intelligence Scale-III (WAIS-III). Two subtests are especially useful in this context, although the entire WAIS-III should be administered to determine if there is significant scatter among the various subtests, rather than relying upon a single subtest result. The most relevant subtests of the WAIS-III are the Digit Symbol subtest and the Block Design subtest. ${ }^{\left[{ }^{66]}\right.}$ The Wechsler 
Memory Scale (Revised) provides a comprehensive assessment of memory function. These psychological testing materials are available, depending upon professional qualifications, from the Australian Council for Educational Research and the Psychological Corporation.

\section{Issues in assessing for cognitive impairment}

- Weighing the client's risk factors for cognitive dysfunction. For example, if the client is 45 years old and has been drinking to excess for 15 years or more, and also reports social or vocational disnuption, there is a risk that this person has some alcohol-related brain damage or cognitive dysfunction. Many clients can report that there has been a decline in memory function as shown by a number of changes in memory ability, although some commentators caution against relying upon the self-report of clients, especially in the case of damage to the frontal lobes, as they may not be aware that the changes have occurred. ${ }^{[8]}$

- If the clinician suspects that the client has marked cognitive impairment, referral to a clinical psychologist or neuropsychologist for further testing might be appropriate.

\section{ASSESSING PHYSICAL WELL-BEING}

The following areas should be assessed:

- medical history

- current physical symptoms

- use of medication

- any current withdrawal symptoms

- liver disease

- previous or current health problems related to drinking

Medical examination should at least assess signs of liver disease, blood pressure, withdrawal symptoms, and organic brain damage. ${ }^{[8]}$ The medical results provide tangible evidence of the effects of risky drinking. There is potential value in the simple act of feeding back to the client the results of the medical examination. For instance, a discussion with the client about the implications of abnormal liver function tests is not only informative, but might also increase the client's motivation to change.

The Drinker's Check-up relies heavily on this motivating function of feeding back objective information. ${ }^{[8]}$

The advantages of feedback are less clear when the medical tests show normal results. However, the whole assessment process should allow clients to assess accurately the degree of their alcoholrelated problems and normal medical results should not detract from this process. The issue of normal range results can be looked at within the context of a clinical interaction and is further discussed in the motivational interviewing material in Chapter 8: Psychosocial interventions.

\section{ASSESSING FAMILY FACTORS}

Clients should be encouraged to explore relevant family issues during assessment. These might include:

- How their drinking might have affected relationships with their spouse or partner, their parents, 
their children, and other significant people in their lives. The Alcohol Problems Questionnaire has a subscale assessing family problems and one assessing marital/relationship problems. ${ }^{[21]}$

- The quality of various family relationships independent of any attributions about the effects of the client's drinking.

- Experience of domestic violence and sexual abuse from the perspective of the client as perpetrator and/or victim. Because of the sensitivity of these issues, it is not usually appropriate to raise them in the first contact session. As mentioned above, these issues may require specialist assessment and intervention.

- The family's role in convincing the client to seek help. A client who is "self-referred" may be responding to family pressure and this is important information for the assessment of the client's motivations and ambivalence.

When it is possible the clinician should interview the spouse or the family members. The interview should provide family members with the opportunity to explore:

- Their observations about the drinker's behaviour.

- The problems they have had in coping with the drinking behaviour. The clinician will need to evaluate the levels of distress within the family, feelings of isolation and confusion, specific crises preceding help-seeking, and who feels responsible for solving the family problems.

- Expectations that family members have about treatment. If the spouse or significant other is going to be involved in the alcohol treatment (see Chapter 8: Psychosocial interventions), the clinician needs to assess whether the couple have adequate communication to enable mutual problem solving.

- What happens before and after drinking episodes, so that particular dynamics relevant to the drinking can be identified. If the spouse's role in therapy is aimed at selectively reinforcing certain behaviours in their partner, then the clinician should be sure that does not threaten the spouse's well-being by reinforcing the notion that she or he is somehow responsible for the partner's drinking.

The family interview is an opportunity for family members to ask questions and to voice their concerns. It is also a good time to help the family see the drinking problem in perspective. For instance, family members should be advised that achieving abstinence or moderation does not necessarily resolve family problems, and that their personal health and wellbeing does not necessarily depend upon the resolution of the drinker's problem. Most importantly, the attitude of the clinician should permit the significant other to help themselves rather than feeling obligated to help the drinker. 
Client involvement in goal setting and treatment planning

III-1

helps increase motivation and retention in treatment.

The stages of change model and its associated questionnaire

IV

(the RTCQ) have not demonstrated consistent reliability and validity. Although the client's readiness to change is important, its assessment may best be carried out through direct questioning.

A number of validated scales are available to assess the client's severity of dependence. Three useful scales are the SADQ-C, the SADD and the ADS.

The Timeline Followback Method helps to obtain an accurate, retrospective account of alcohol consumption over a particular period, typically three months.

Psychological comorbidity, particularly anxiety and depression, is common in alcohol dependent clients.

Including family members in the assessment can provide important information about the triggers for drinking, drinking patterns, and alcohol-related problems. It also provides an opportunity to clarify family members' expectations about treatment.

Assessment for more intensive intervention should be conducted in a semi-structured, narrative style, with strategic use of structured techniques and questionnaires such as the Timeline Followback Method for assessing alcohol intake, and the SADD or the SAD-Q for assessing severity of dependence.

The length of the assessment process needs to be balanced with retaining the client in treatment. Therefore, clinicians need to make decisions about which aspects of assessment are essential. Key topics are the client's consumption of alcohol, level of alcohol dependence, cognitive functioning, psychological comorbidity, family situation, physical wellbeing, and readiness for change.

Assessment should lead to a clear, mutually acceptable treatment plan that structures a specific intervention to meet the client's needs.

Readiness to change may be best assessed through direct questioning Where possible, and with the client's permission, family members should be involved in the assessment process

Assessment for anxiety and depressive symptoms and disorders should be routine.

Strong

Moderate

Moderate

Strong 
Chapter 4: Patient-treatment matching 


\section{Chapter 4: Patient-treatment matching}

\section{Chapter aim}

The aim of this chapter is to describe how clinicians can decide which treatment intervention is likely to be most effective for a patient, based on the individual characteristics and needs of that patient.

Patient-treatment matching refers to the prescription of treatment according to individual patient characteristics, as opposed to providing the same therapy to all patients with a common diagnosis.

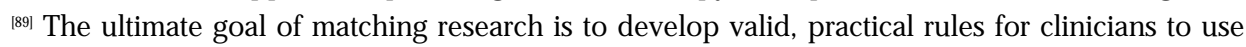
in assigning patients to treatment regimes. In the accompanying literature review, a range of treatment options were evaluated in terms of the evidence relating to their effectiveness. A major conclusion of this review was that there are a range of treatment interventions, all of which appear to have a role in treatment, but that no single intervention is effective for all persons with alcohol problems. A second major conclusion is that there is no reason to believe that persons with alcohol problems are a homogeneous group with identical reasons for risky drinking, or that they all should receive a single intervention of the same type, content and duration.

Despite the intuitive appeal of these conclusions, the largest investigation of patient-treatment matching, Project MATCH, found no evidence to support the patient-treatment matching hypothesis.

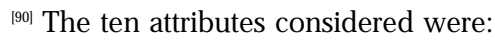

- severity of alcohol involvement

- cognitive impairment

- client conceptual level

- gender

- meaning seeking

- motivational readiness to change

- psychiatric severity

- social support

- sociopathy

- typology

Psychiatric severity was the only attribute that showed a significant attribute by treatment interaction: in the outpatient study, clients low in psychiatric severity had more abstinent days after 12-step facilitation than after cognitive behavioural therapy. Neither treatment was clearly superior for clients with higher levels of psychiatric severity.

Although evidence does not indicate that certain patients respond best to certain treatment interventions, in practice clinicians do match clients to treatments. Clinicians often use criteria such as severity of dependence, the presence of co-occurring pathology or other problems such as marital problems or a lack of social support to assign patients to treatment. By doing this, clinicians ensure that the treatments offered consider the factors associated with the alcohol problem. ${ }^{199}$ This form of matching depends upon the careful and comprehensive assessment described in Chapter 3: Screening and assessment. 
Further, the clinician can make the decision with the client about treatment modality. It is thought that if the client contributes in deciding which treatment to receive, they are more likely to participate more fully in treatment. In this situation the clinician describes the available treatment options that might best assist with the various deficits or problems identified as being associated with the client's drinking, and the client and clinician agree upon which treatment is likely to be most appropriate.

\section{SUMMARY}

The role of matching in alcohol treatment is an important area of research, but at this stage research evidence provides few clear guidelines. In the absence of clear research evidence, clients and clinicians should make joint decisions about the treatment likely to be most appropriate, given the severity of dependence of the client, and their treatment goals.

\section{SUIMIMARY OF EVIDENCE: PATIENTT-TREATMENT MATCHING}

Evidence from well-controlled trials does not support the patient-treatment matching hypothesis

\section{LEVEL OF EVIDENCE}

II

RECOMMIENDATIONS: PATIENTT-TREATMENT MATCHING

STRENGTH OF RECOMMENDATION

The intensity of interventions should vary, with clients with more severe problems receiving more intensive treatments

Comorbid psychological disorders should be taken into account when assigning patients to treatment, particularly when pharmacotherapies are being considered as a relapse prevention strategy.

The clinician and the client should discuss and agree upon treatment goals and interventions.
Strong

Strong

Moderate 
Chapter 5: Alcohol withdrawal management 


\section{Chapter 5: Alcohol withdrawal management²}

The clinical management of alcohol dependent drinkers during withdrawal is a critical phase of an alcohol treatment program, since it serves to minimise the severity of withdrawal symptoms that occur when alcohol consumption is ceased.

\section{CHAPTER AIM}

To provide knowledge and guidance on the appropriate management of patients in alcohol withdrawal.

\section{Alcohol withdrawal syndrome}

People who are physically dependent upon alcohol are likely to experience withdrawal symptoms 6 to 24 hours after the last drink is consumed. The alcohol withdrawal syndrome usually begins as the blood alcohol level (BAL) reduces and may become clinically apparent usually before the BAL reaches zero. ${ }^{[1,192]}$ The alcohol withdrawal is usually self-limiting and usually uncomplicated resolving within 5 days with minimal or no intervention. However, this depends largely on the individual's drinking pattern, frequency, duration and quantity. While for most individuals the alcohol withdrawal syndrome is short-lived and inconsequential in others it increases in severity through the first 48 to 72 hours of abstinence. The patient becomes highly vulnerable to psychological and physiological stress during this time. Alcohol withdrawal seizures may occur 12 to 48 hours post-drinking and alcohol withdrawal delirium tremens 48 to 96 hours post-drinking. ${ }^{\text {[93] }}$ Figure 5.1 outlines the alcohol withdrawal syndrome progression.

\section{FIGURE 5.1:THE PROGRESSION OFTHE ALCOHOL WITHDRAWAL SYNDROME}

Source: Frank

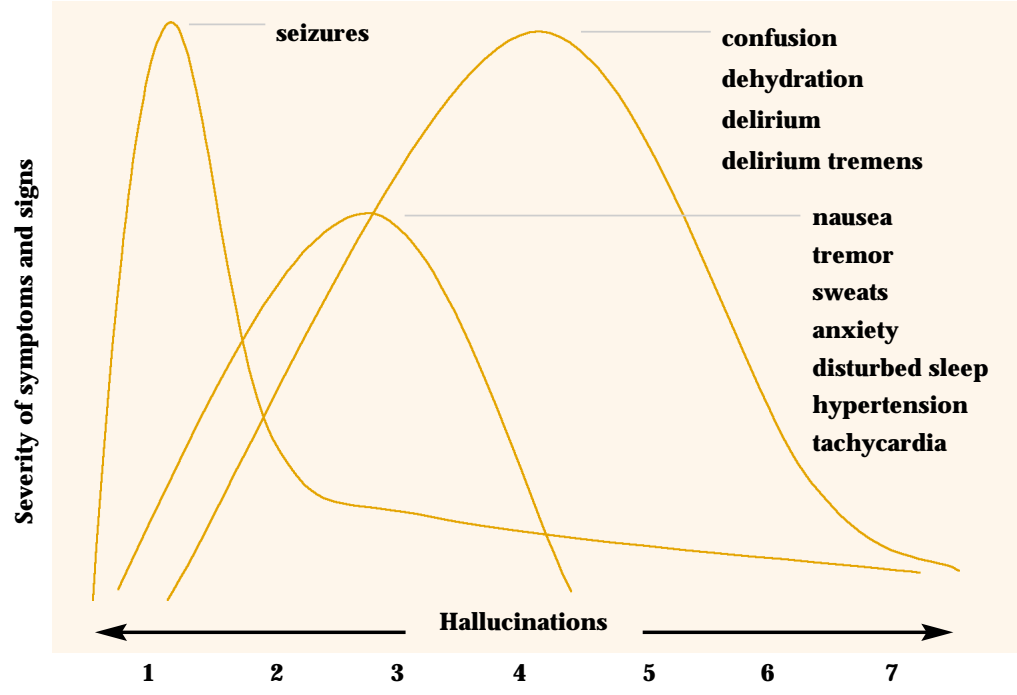

and Pead (1995). Reproduced with permission. ${ }^{[94]}$

2 Since the 1993 monograph 'An outline for the management of alcohol problems: quality assurance project',there has been little research on alcohol withdrawal. Therefore with permission from the authors the withdrawal section is based upon that of the previous monograph. 
Alcohol withdrawal symptoms may be present when the person has a significant BAL. The BAL does not have to be zero for the onset of alcohol withdrawal to occur, with a significant proportion of dependent drinkers experiencing the onset of withdrawal symptoms before the BAL reaches zero. Patient care should not be decided based on the BAL alone, the alcohol withdrawal rating scales should also be used to assess the patient's level of alcohol withdrawal symptoms.

The severity of alcohol withdrawal also depends on the number of drinks per day, the total number and severity of past withdrawal episodes, greater use of non-therapeutic sedative-hypnotic drugs, and the number of medical problems. However, caution is necessary as clinical experience suggests that some patients may undergo severe withdrawal at lower levels of consumption than others who are heavier drinkers. Therefore it is important to monitor patients carefully during the alcohol withdrawal period to identity patients at risk. Patients withdrawing from alcohol may also be withdrawing from multiple substances. As a result these patients may require closer monitoring for abstinence on a range of substances in addition to alcohol.

\section{ALCOHOL WITHDRAWAL SYMPTOMS AND SIGNS}

THE SIGNS AND SYMPTOMS OFTHE ALCOHOLWITHDRAWAL SYNDROME INCLUDE:

- increased body temperature (hyperthermia)

- increased pulse rate (tachycardia)

- increased respiration rate

- increased blood pressure (hypertension)

- nausea and vomiting

- tremor

- sweating

- agitation and anxiety

- disturbed sleep

- tactile disturbances such as pins and needles, itching, burning, numbness, crawling sensations and "electric fleas", including tactile hallucinations

- auditory and/or visual disturbances including hallucinations 


\section{Medical care for alcohol withdrawal complications}

\section{All health professionals planning on prescribing or using any of the medications listed below are advised to first refier to MIIMS Australia.. ${ }^{[95]}$}

\section{SEIZURES}

Withdrawal-induced seizures may occur 12 to 48 hours after the last drink is consumed. A patient with a previous history of alcohol withdrawal seizures should be monitored regularly and carefully with an alcohol withdrawal rating scale and withdrawal medication given, preferably as a loading dose regime with diazepam orally and in some cases intravenously. However, one study has examined the use of $2 \mathrm{mg}$ lorazepam in $2 \mathrm{ml}$ of normal saline compared to $4 \mathrm{ml}$ of normal saline for preventing recurent seizures in patients withdrawing from alcohol and found that the lorazepam group had a significant effect on preventing recurrent seizures. ${ }^{\text {[9] }}$ If the patient experiences two or more seizures, focal seizures ${ }^{\left[{ }^{97]}\right.}$ or develops status epilepticus the patient should be investigated separately since these are not necessarily because of alcohol withdrawal. ${ }^{\text {[9] }}$

\section{HALLUCINATIONS}

Some patients may experience hallucinations, which may occur during any stage of the alcohol withdrawal phase.

The symptoms and signs of hallucinations include:

- hallucinations usually auditory

- perceptual distortions

- paranoid delusions

- psychomotor disturbances

- abnormal affect

Patients experiencing hallucinations should be monitored regularly and carefully. Alcohol withdrawal medication such as diazepam should be given. However, if the patient continues to experience hallucinations an anti-psychotic medication, such as haloperidol, may be given as well. An initial dose of $2.5 \mathrm{mg}$ to $10 \mathrm{mg}$ of haloperidol orally or intramuscularly can be given if diazepam is not effective in reducing or alleviating the hallucinations. Additional doses of haloperidol may be given if the patient continues to experience hallucinations. ${ }^{[1,9]}$ The patient should also be monitored carefully for hypotension.

\section{DELIRIUM}

A small percentage of patients withdrawing from alcohol experience delirium during the first 24 to 48 hours of withdrawal.

The symptoms and signs of delirium include:

- agitation

- hyperactivity

- tremor

- confusion

- disorientation

Patients experiencing delirium should be monitored regularly and carefully. Alcohol withdrawal medication such as diazepam and fluids should be given to prevent progression to delinium tremens. 


\section{DELIRIUM TREMENS (DTS)}

Delirium tremens is by far the most serious life-threatening complication of the alcohol withdrawal syndrome, which usually occurs 48 to 96 hours after the last drink is consumed, and should be monitored.

The symptoms and signs of delirium tremens include:

- exaggerated alcohol withdrawal syndrome

- extreme restlessness or agitation

- autonomic instability

- gross tremor

- confusion and disorientation

- paranoid ideation

- hallucinations (any senses)

Delirium tremens complications may include dehydration, arrhythmias, hypotension, renal failure and pneumonia. Patients with DTs should be monitored and given intravenous fluids, effective sedation (either benzodiazepines or major tranquillisers) and should be treated for conditions occurning at the same time. Management needs to be tailored to the patients' needs, however an intravenous injection of 10 to $20 \mathrm{mg}$ diazepam may be given over two to five minutes. ${ }^{[1,97]}$ The patient should be observed continuously and if necessary a further two doses (given over the next hour). ${ }^{[1,97]} \mathrm{A}$ maintenance dose of one to five $\mathrm{mg}$ per hour (gradually reducing) intravenously may be necessary over the first 24 hours or 10 to $20 \mathrm{mg}$ diazepam every two hours for six hours as required followed by 10 to $20 \mathrm{mg}$ every six hours until 24 hours since initial dose. ${ }^{[1,9]}$ The patient should be kept under close supervision at all times.

\section{WERNICKE-KORSAKOFF'S SYNDROME}

Wernicke-Korsakoff's syndrome is a form of brain injury resulting from a lack of thiamine (vitamin B1) deficiency in chronically alcohol dependent patients. The condition could lead to permanent brain damage and memory loss if not treated early.

The symptoms and signs of Wernicke-Korsakoff's syndrome:

- nystagmus (reduced eye movement)

- neuropathy

- ataxia

- confusion

Patients at risk of Wemicke-Korsakoff's syndrome should be treated with 100mg of intramuscular or oral thiamine before any glucose intake.[1] 


\begin{tabular}{|c|c|}
\hline SUMMARY OF EVIDENCE: ALCOHOL WITHDRAWAL & LEVEL OF EVIDENCE \\
\hline $\begin{array}{l}\text { It is important to monitor patients for alcohol } \\
\text { withdrawal symptoms. The severity of the symptoms } \\
\text { depends upon the number of drinks per day, } \\
\text { duration of drinking, number of previous alcohol } \\
\text { withdrawal episodes, a known coexisting illness and } \\
\text { use of other psychotropic drugs. }\end{array}$ & I \\
\hline $\begin{array}{l}\text { Alcohol withdrawal complications may occur, such } \\
\text { as seizures, hallucinations, delirium and delirium } \\
\text { tremens. Patients should be monitored with an } \\
\text { alcohol withdrawal rating scale and managed with } \\
\text { withdrawal medication. }\end{array}$ & I \\
\hline $\begin{array}{l}\text { Alcohol dependent patients risk developing } \\
\text { Wernicke-Korsakoff's syndrome (permanent } \\
\text { brain damage), which can be prevented by giving } \\
\text { the patient parenteral thiamine before any glucose } \\
\text { intake during withdrawal. }\end{array}$ & I \\
\hline RECOMMENDATIONS: ALCOHOL WITHDRAWAL & STRENGTH OF RECOMMENDATIONS \\
\hline $\begin{array}{l}\text { Patient experiencing withdrawal complications such } \\
\text { as seizures, hallucinations, delirium and delirium } \\
\text { tremens should be monitored carefully and regularly } \\
\text { with a withdrawal rating scale and withdrawal } \\
\text { medication should be given. }\end{array}$ & Strong \\
\hline $\begin{array}{l}\text { Patients at risk of Wernicke-Korsakoff's syndrome should } \\
\text { be treated with } 100 \mathrm{mg} \text { of intramuscular or oral thiamine } \\
\text { before any glucose intake. }\end{array}$ & Strong \\
\hline
\end{tabular}




\section{Monitoring the withdrawal state}

All patients in alcohol withdrawal, or who are considered at risk of alcohol withdrawal, should be monitored regularly. It is beneficial to use an alcohol withdrawal rating scale to assess the severity of the withdrawal state.

\section{- The alcohol withdrawal rating scales are intended for monitoring the severity of the alcohol withdrawal syndrome and should not be used as diagnostic tools.}

The Clinical Institute Withdrawal Assessment for Alcohol revised CIWA-Ar is a 10-item scale and is helpful in assessing the severity of the alcohol withdrawal (Table 5.1). It helps to guide treatment and allows clinicians to communicate more objectively about the severity and management of alcohol withdrawal. The CIWA-Ar is important in helping to indicate when the alcohol withdrawal is continuing to develop in spite of conservative measures. Patients scoring more than 10 have an increased risk of complications if they remain without sedatives. ${ }^{[99}$ As the CIWA-Ar score increases the risk increases and the patient should be monitored closely and sedatives given. The scale is usually used one to three times a day and more frequently when patients are symptomatic. ${ }^{[98]}$

An alternative scale is the Alcohol Withdrawal Symptoms - Rating Scale (AWS) (Table 5.2). The AWS has not been validated. However, the AWS is widely used and is considered acceptable for use in hospitals and non-medicated environments despite no strong evidence or validation. If the CIWA-Ar scale is not available then an AWS score of up to four indicates a mild withdrawal syndrome, five to seven a moderate withdrawal, eight to 14 a severe withdrawal and 15 or more is indicative of a very severe withdrawal. Close monitoring is advised at least every four hours and if the AWS score reaches 10 or more, every two hours. The point at which sedatives should be prescribed depends upon the severity of the alcohol withdrawal and the setting in which the alcohol withdrawal is taking place.

The Short Alcohol Withdrawal Scale (SAWS) was developed as a self-completion scale (Table 5.3). The SAWS should be explained to the patient on first administration. The scale is designed for administration once every 24 hours. The severity of each symptom is rated on a four point scale from none (zero) to severe (four). An item score total of 12 or above indicates the possible need for medication to reduce alcohol withdrawal symptoms. The SAWS requires further research and is recommended for use with the standard clinical assessment and nursing observations.

Not all patients will be withdrawing from just alcohol. Patients may also be withdrawing from other substance(s) such as heroin and diazepam. In these cases, the patient should be monitored very closely for withdrawal from alcohol and other substance(s). Clinicians are advised to refer to the withdrawal guidelines for these substance(s) and use these guidelines with these alcohol withdrawal management guidelines. 


\section{TABLE 5.1: CLINICAL INSTITUTE WITHDRAWAL ASSESSMENT FOR ALCOHOL REVISED (CIWA-AR)}

\section{NAUSEAANDVOMITING}

Ask "Do you feel sick to your stomach? Have you vomited?" and observe.

q 0 No nausea and no vomiting

q 1 Mild nausea with no vomiting

q 2

9 3

q 4 Intermittent nausea with dry heaves

q 5

व 6

q 7 Constant nausea, frequent dry heaves and vomiting

\section{TREMOR}

Observe patient's arms extended and fingers spread apart

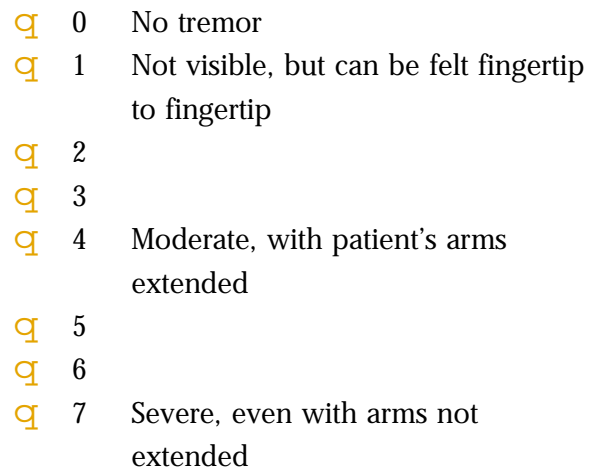

\section{PAROXYSMAL SWEATS}

$\begin{array}{lll}\Upsilon & 0 & \text { No sweat visible } \\ \Upsilon & 1 & \text { Barely perceptible sweating, palms } \\ & & \text { moist } \\ \Upsilon & 2 & \\ \Upsilon & 3 & \\ \Upsilon & 4 & \text { Beads of sweat obvious on forehead } \\ \Upsilon & 5 & \\ \Upsilon & 6 & \\ \Upsilon & 7 & \text { Drenching sweats }\end{array}$

ANXIETY

Observe, and ask "Do you feel nervous?'

$$
\begin{array}{lll}
\mathrm{q} & 0 & \text { No anxiety, at ease } \\
\mathrm{q} & 1 & \text { Mildly anxious } \\
\mathrm{q} & 2 & \\
\mathrm{q} & 3 & \\
\mathrm{q} & 4 & \text { Moderately anxious, or guarded, so } \\
& & \text { anxiety is inferred } \\
\mathrm{q} & 5 & \\
\mathrm{q} & 6 & \\
\mathrm{q} & 7 & \text { Equivalent to acute panic states as } \\
& & \begin{array}{l}
\text { seen in severe delirium or acute } \\
\end{array} \\
& & \text { schizophrenic reactions }
\end{array}
$$

\section{AGITATION \\ q 0 Normal activity \\ q 1 Somewhat more than nomal activity \\ व 2 \\ 9 3 \\ q 4 Moderately fidgety and restless \\ q 5 \\ व 6 \\ 9 7 Paces back and forth during most of the interview, or constantly thrashes about}

\section{TACTILE DISTURBANCES}

Ask "Have you any itching, pins and needles sensations, any buming, any numbness or do you feel bugs crawling on or under your skin?'

\section{q 0 None}

q 1 Very mild itching, pins and needles, burning or numbness

q 2 Mild itching, pins and needles, burning or numbness

93 Moderate itching, pins and needles, burning or numbness

q 4 Moderately severe hallucinations

q 5 Severe hallucinations

9 $6 \quad$ Extremely severe hallucinations

q 7 Continuous hallucinations 


\section{TABLE 5.1: CLINICAL INSTITUTE WITHDRAWAL ASSESSMENT FOR ALCOHOL REVISED (CIWA-AR) CONT.

\begin{tabular}{lll}
\hline \multicolumn{2}{l}{ AUDITORY DISTURBANCES } \\
\hline$q$ & 0 & Not present \\
$q$ & 1 & Very mild harshness or ability to \\
& & frighten \\
$q$ & 2 & Mild harshness or ability to frighten \\
$q$ & 3 & Moderate harshness or ability to \\
& & frighten \\
$q$ & 4 & Moderately severe hallucinations \\
$q$ & 5 & Severe hallucinations \\
$q$ & 6 & Extremely severe hallucinations \\
$q$ & 7 & Continuous hallucinations
\end{tabular} \\ VISUAL DISTURBANCES \\ Ask "Does the light appear to be too bright? \\ ORIENTATION AND CLOUDING OF SENSORIUM \\ Ask "What day is this? Where are you? Who am I?' \\ q 0 Orientated and can do serial additions \\ q 1 Cannot do serial additions or is uncertain about date \\ 2 Disorientated for date by no more than 2 calendar days \\ q 3 Disorientated for date by more than 2 calendar days \\ q 4 Disorientated for place and/or person} Is its colour different? Does it hurt your eyes? Are you seeing anything that is disturbing to you? Are you seeing things you know are not there?' and observe.

$\begin{array}{lll}q & 0 & \text { Not present } \\ q & 1 & \text { Very mild sensitivity } \\ q & 2 & \text { Mild sensitivity } \\ q & 3 & \text { Moderate sensitivity } \\ q & 4 & \text { Moderately severe hallucinations } \\ q & 5 & \text { Severe hallucinations } \\ q & 6 & \text { Extremely severe hallucinations } \\ q & 7 & \text { Continuous hallucinations }\end{array}$

HEADACHES, FULLLNESS IN HEAD

Ask "Does your head feel different? Does it feel like there is a band around your head?" Do not rate for dizziness or light-headedness. Otherwise, rate severity.

$\begin{array}{lll}q & 0 & \text { Not present } \\ q & 1 & \text { Very mild } \\ q & 2 & \text { Mild } \\ q & 3 & \text { Moderate } \\ q & 4 & \text { Moderately severe } \\ q & 5 & \text { Severe } \\ q & 6 & \text { Very severe } \\ q & 7 & \text { Extremely severe }\end{array}$

Source: Sullivan et al. (1989). ${ }^{\text {[99] }}$ 


\section{ITEM 1 PERSPIRATION}

q 0 No abnormal sweating.

q 1 Moist skin.

q 2 Localised beads of sweat (such as on face, chest etc).

q 3 Whole body wet from perspiration.

q 4 Profuse sweating (such as Clothes, linen etc are wet).

\section{ITEM 2 TREMOR}

q 0 No tremor.

q 1 Slight intentional tremor.

q 2 Constant slight tremor of upper extremities.

q 3 Constant marked tremor of extremities.

\section{ITEM 3 ANXIETY}

q 0 No apprehension of anxiety.

q 1 Slight apprehension.

q 2 Apprehension or understandable fear (such as of withdrawal symptoms).

q 3 Anxiety occasionally accentuated to a state of panic.

q 4 Constant panic-like anxiety.

\section{ITEM 4 AGITATION}

q 0 Rests normally during day, no signs of agitation.

q 1 Slight restlessness, cannot sit or lie still, awake when others sleep.

q 2 Moves constantly, looks tense, wants to get out of bed but obeys requests to stay in bed.

q 3 Constantly restless, gets out of bed for no obvious reason, returns to bed if taken.

q 4 Maximally restless, aggressive, ignores request to stay in bed.
ITEM 5 AXILLA (ARMPIT)TEMPERATURE

q $0 \quad$ Temperature of $370 \mathrm{c}$ or less

q 1 Temperature of $37.1^{\circ \mathrm{c}}$ to $37.5^{\circ \mathrm{cc}}$

q 2 Temperature of $37.6^{\mathrm{oc}}$ to $38.0^{\mathrm{oc}}$

q 3 Temperature of $38.1^{\circ \mathrm{c}}$ to $38.5^{\mathrm{oc}}$

q 4 Temperature above $38.5^{\text {oc }}$

ITEM 6 HALLUCINATIONS (A FALSE PERCEPTION OF SIGHT, SOUND, TASTE ORTOUCH)

q $\quad 0 \quad$ No evidence of hallucinations.

q 1 Distortions of real objects. Aware that these are not real if this is pointed out.

2 Appearance of totally new objects or perceptions, aware that these are not real if this is pointed out.

3 Believes the hallucinations real but still oriented in place and person.

4 Believes self to be in a totally nonexistent environment, preoccupied and cannot be diverted or reassured.

\section{ITEM 7 ORIENTATION}

q 0 The patient is fully oriented in time, place and person.

q 1 The patient is oriented in person but is not sure where he is or what time it is.

q 2 Oriented in person but disoriented in time and place.

q 3 Doubtful personal orientation, disoriented in time and place, there might be short periods of lucidity.

q 4 Disoriented in time, place and person, no meaningful contact can be obtained.

Source: Novak et al (1989). ${ }^{[100]}$ 


\section{TABLE 5.3:THE SHORT ALCOHOL WITHDRAWAL SCALE (SAWS)}

Please put a tick in the boxes to show how you have been feeling for all of the following conditions in the last 24 hours.

$\begin{array}{lcccc} & \text { None (0) } & \text { Mild (1) } & \text { Moderate (2) } & \text { Severe (3) } \\ \text { Anxious } & q & q & q & q \\ \text { Sleep disturbance } & q & q & q & q \\ \text { Problems with memory } & q & q & q & q \\ \text { Nausea } & q & q & q & q \\ \text { Restless } & q & q & q & q \\ \text { Tremor (shakes) } & q & q & q & q \\ \text { Feeling confused } & q & q & q & q \\ \text { Sweating } & q & q & q & q \\ \text { Miserable } & q & q & q & q \\ \text { Heart pounding } & q & q & q & q\end{array}$

Source: Gossop et al (2002). Reproduced with permission. ${ }^{1011}$

\section{SUMMARY OF EVIDENCE: WITHDRAWAL SCALES}

The Clinical Institute Withdrawal Assessment for Alcohol Revised (CIWA-Ar) is the most widely used and validated alcohol withdrawal scale.

However, the expert panel agreed that the Alcohol Withdrawal Scale - Rating Scale (AWS) is widely used and is acceptable for use in hospitals and non-medicated environments despite no strong evidence or validation.

The Short Alcohol Withdrawal Scale (SAWS) is a more recent scale to be used with the standard clinical assessment and observation.

\section{LEVEL OF EVIDENCE}

I

IV

IV

STRENGTH OF RECOMMENDATIONS

Strong

Patient withdrawing from alcohol should be monitored with a withdrawal rating scale. The CIWA-Ar is the most validated and recommended scale to use.

If the CIWA-Ar scale is unavailable then the AWS should be used, however this scale is not validated despite it wide use in Australia.

All of the alcohol withdrawal rating scales are to be used Moderate to monitor the severity of the withdrawal syndrome and are not intended as diagnostic tools. 


\section{Home-based, outpatient and inpatient withdrawal management}

A range of alcohol withdrawal management settings currently exist. The appropriateness of each of these settings to an individual drinker's case will depend upon good clinical judgement of the actual or likely severity of the alcohol withdrawal syndrome; the presence of other physical and psychiatric conditions; and the choice made by the drinker. A "triage" system with clear criteria known by all staff for the different alcohol withdrawal management options should be in place to screen all patients presenting for alcohol withdrawal management.

These withdrawal management settings include:

- Home-based withdrawal is supervised medically or non-medically, which is conducted under supervision.

- Outpatient withdrawal is supervised medically or non-medically, which is conducted under supervision.

- Inpatient withdrawal deals with medical emergencies associated with alcohol withdrawal, such as delirium tremens.

Home-based, outpatient and inpatient withdrawal management settings are not for alcohol withdrawal management alone. The majority of these settings will provide withdrawal management for a range of substances such as heroin and diazepam. Therefore, staff training is required to detect withdrawal symptoms from other substances besides alcohol.

\section{HOME-BASED WITHDRAWAL MANAGEMENT}

Many people who choose to withdraw from alcohol can be managed in the home. Home-based withdrawal management involves the patient withdrawing from alcohol at home in a supportive setting or group accommodation, such as a hostel or halfway house. Patient's withdrawal symptoms are usually monitored by visits from a health care worker and telephone calls. Medications are usually managed by the patient or lay carer and the through access to a 24-hour telephone support line.

Home-based withdrawal management may be appropriate for those who are likely to suffer from mild to moderate alcohol withdrawal, may not require sedative medication, have no known coexisting medical or psychiatric history, and for groups of people who may have difficulty reaching inpatient withdrawal settings, such as women who have children at home, or people from cultural groups who value intensive family or community support that cannot be readily provided by residential settings.

Alcohol withdrawal management at home may be attempted provided that:

- There are no signs of severe alcohol withdrawal, such as severe tremor, tachycardia greater than 110 beats per minute, seizures, delirium, clouding of consciousness or hallucinations at the time.

- There is no history of severe alcohol withdrawal, such as delirium tremens or alcohol withdrawal seizures.

- There are supportive relatives or friends who could stay with the patient during the period of alcohol withdrawal, supervise administration of medication and call for medical assistance if it becomes necessary. 
- There are no other significant medical illnesses, such as pneumonia or pancreatitis. Delirium tremens is more common when such illnesses are present.

- There is no evidence of suicidal ideation, or severe depressive disorder.

- The patient does not have ready access to a supply of alcohol or other drugs at home.

There are some potential negative aspects to home-based withdrawal management. Mainly, the failure rate of home-based withdrawal management may be higher than for inpatient withdrawal management. The reasons for this include:

- Patients withdrawing at home are less likely to be removed from the factors that have become associated with regular drinking.

- Patients are thus less likely to complete the withdrawal process without drinking alcohol.

- It is not appropriate for some drinkers, for example, the homeless and those patients at risk of severe alcohol withdrawal.

Readers are referred to the "Guide to home detoxification" by Saunders et al. (1996) ${ }^{[102]}$ for further information on home-based withdrawal management.

\section{OUTPATIENT WITHDRAWAL MANAGEMENT}

Outpatient withdrawal management is similar to home-based withdrawal management; however the patient attends a clinic or outpatient withdrawal management setting for observation, assessment by trained staff and to collect alcohol withdrawal medication usually on a daily basis.

Outpatient withdrawal management, like home-based withdrawal management may be appropriate for those who are likely to suffer from mild to moderate alcohol withdrawal, are not in need of sedative medication, have no known coexisting medical or psychiatric history, and for groups of people who may have difficulty attending inpatient services. The criteria for outpatient withdrawal management are similar to home-based withdrawal management as discussed above, except that the "support of family and friends" is not necessary because unlike home-based withdrawal management, outpatient withdrawal management allows for close observation, assessment by staff and access to other alcohol treatment services such as counselling. The main negative aspect of outpatient withdrawal management is the possibility of relapse because the patient's environment remains the same, such as in the homeless.

\section{INPATIENT WITHDRAWAL MANAGEMENT}

Inpatient withdrawal management settings may be a community residential setting or a dedicated acute hospital bed with well-trained clinicians available for the care of dependent drinkers at risk of alcohol withdrawal complications. Community residential settings are different to acute hospital beds, in that they are more domestic/home like environment than clinical, patients are ambulatory and are not lying in bed all day, patients are either supervised medically or non-medically, and community residential settings may provide group programs focusing on, for example relapse prevention, how to cope with symptoms, and stress management. 
Circumstances where inpatient withdrawal management is indicated include; medical or psychiatric disorders, unsafe environment, homelessness, living with other addicted individuals, and/or a history of failed attempts to abstain in either a home-based or outpatient withdrawal setting. Admission to inpatient withdrawal management settings should be based on meeting at least one of the criteria outlined below.

Inpatient withdrawal management criteria are set out below:

- A history of severe alcohol withdrawal symptoms or episodes such as seizures, recurrent alcohol withdrawal seizures, delirium or delirium tremens that should be managed in a residential or inpatient basis.

- The probability of imminent and severe alcohol withdrawal syndrome, or else the actual presence of a marked alcohol withdrawal state indicating the need for inpatient withdrawal management or residential care.

- The presence of a known coexisting psychiatric or medical disorder, such as depression, suicidal ideation, chest infection, pneumonia, or pancreatitis -each of which increase the likelihood that the alcohol withdrawal will end in injury or death.

- A home environment that is unfavourable to successful alcohol withdrawal, because of a lack of supervision or because of the presence of heavy drinkers and alcohol that predisposes the withdrawing drinker to relapse.

- The lack of a suitably supervised home-based or outpatient withdrawal management settings.

However, inpatient withdrawal management may face some issues such as a lack of resources, difficulties in managing patient care, occupational health and safety issues for staff, patients and visitors, dealing with patient welfare issues, policing patient access to alcohol and other substances and the cost of running an inpatient withdrawal management setting.

Overall, home-based, outpatient and inpatient withdrawal management not only offers alcohol withdrawal management but also provides advice, support and allows patients to be referred to ongoing treatment such as counselling and accommodation. 
Home-based withdrawal management is appropriate

when there are no signs of severe withdrawal, no history of severe withdrawal, there are supportive relatives or friends who can help to assist and look after the person and there are no known coexisting medical or psychiatric illnesses.

Outpatient withdrawal management patients attend II a clinic or facility, usually on a daily basi,s for assessment and to collect withdrawal medication. It is appropriate for patients with no obvious se vere withdrawal, or history of severe withdrawal and no known coexisting medical or psychiatric illnesses.

Inpatient withdrawal management is required for people who have a severe history of alcohol withdrawal symptoms, a known coexisting medical or psychiatric illness and/or an unfavourable home environment.

Home-based withdrawal management is Strong recommended for patients with mild to moderate withdrawals who have a support network, no known coexisting medical or psychiatric illness and no history of withdrawal complications.

Outpatient withdrawal management is appropriate Strong for patients with mild to moderate withdrawals who have no history of severe withdrawal or known coexisting medical or psychiatric illness.

Inpatient withdrawal management is required for Strong people who have severe withdrawal complications, a history of withdrawal complications or a known coexisting medical or psychiatric history. 


\section{Supervised medicated and non-medicated withdrawal management}

Alcohol withdrawal can be managed in either a supervised medicated by substituting a controlled sedation for uncontrolled alcohol intoxication, or in a supervised non-medicated withdrawal management setting.

Outlined below are the two options:

- Supervised non-medicated withdrawal monitors patients who are at risk of relapse but are not at risk of serious alcohol withdrawal. Monitoring may take place in either a home-based, outpatient or inpatient withdrawal management setting.

- Supervised medicated withdrawal treats patients who are at risk of serious alcohol withdrawal, usually in an inpatient withdrawal management setting. However, patients experiencing mild to moderate alcohol withdrawal may be treated in a home-based or outpatient withdrawal management setting.

A supervised non-medicated alcohol withdrawal is appropriate for patients with mild to moderate alcohol withdrawal who have no known coexisting medical or psychiatric disorders. A supervised non-medicated withdrawal may take place in either a home-based, outpatient or inpatient withdrawal management setting. The rationale for this approach is that withdrawal symptoms, including anxiety and agitation, are lessened and may be prevented by supportive counselling in a non-stimulating, non-threatening environment.

\section{- Patients in a supervised non-medicated withdrawall setting should be monitored with an alcohol withdrawal rating scale. If alcohol withdrawal complications become evident the patient should be given withdrawal medicated or transferred to a supervised medicated withdrawal setting.}

The essential elements of a supervised non-medicated withdrawal approach are outlined in Table 5.4. These supervised non-medicated withdrawal management principles are also helpful in the context of medicated withdrawal. 
PRINCIPLES OF SUPERVISED NON-MEDICATED ALCOHOLWITHDRAWAL

- Use a quiet, non-stimulating, non-threatening and safe environment.

- Employ a slow, steady, non-threatening approach to reduce anxiety.

- Explain all interventions clearly.

- Speak slowly and distinctly in a friendly manner.

- Maintain continuous eye contact when speaking.

- Avoid confrontation and arguments.

- Test the patient's reality base and orientation repeatedly and, if necessary, re-acquaint the patient with his or her environment.

- Attempt to explain to the patient that the unreal nature of illusions and hallucinations may cause anxiety and are likely to be part of the alcohol withdrawal syndrome.

- A night light reduces the likelihood of perceptual errors and an exacerbation of anxiety and psychotic phenomena during the night.

- When the patient is endangering his life or threatening others, minimal restraint may be necessary. If coercion is required, give clear directions to the patient.

- Sedation should be considered if restraint is required, or persistent or increasing disorientation, delusions or hallucinations are present.

- Thiamine should be given in all cases, preferably 100mg intramuscularly, or failing that 100 $\mathrm{mg}$ orally three times daily. Administration of other B group vitamins and vitamin $\mathrm{C}$ is advisable.

Source: Mattick and Jarvis (1993). ${ }^{[1]}$

A supervised medicated alcohol withdrawal is indicated for patients who may suffer or are suffering a severe alcohol withdrawal. A supervised medicated alcohol withdrawal usually takes place in an inpatient withdrawal management setting; however in mild to moderate cases a supervised medicated withdrawal may take place in a home-based or outpatient withdrawal management setting.

\section{- Patients in a supervised medicated withdrawal setting should be monitored using a withdrawal rating scale. Withdrawal medications should be provided to prevent and treat withdrawal symptoms and complications.}

The essential principle of a supervised medicated alcohol withdrawal is that the dose of the sedative is titrated against the severity of the patient's alcohol withdrawal syndrome. Drugs should not be given to patients who are still intoxicated. The medication most commonly used for alcohol withdrawal is diazepam (discussed later on in this section). These medications also have the advantage of having some anticonvulsant properties. Medical practitioners and other health professionals are advised to become familiar with these drugs and gain experience on the dosage and duration of treatment necessary for patients with alcohol withdrawal syndromes of different severity. 
Procedural guidelines for the nursing management of patients who are intoxicated or are withdrawing from alcohol have been developed in Australia. ${ }^{1033}$ These guidelines, Alcohol and Other Drugs Policy for Nursing Practice in NSW: Clinical Guidelines, are presented in an easily understood format and are recommended for general dissemination.

At the time of writing draft clinical guidelines were being prepared for nurses and midwives called Alcohol, Tobacco and other drugs: A framework for Policy and Clinical Practice for Nurses and Midwives: Clinical Guidelines 2002-2005 by the Drug and Alcohol Services Council of South Australia and Flinders University.

RECOMMENDATIONS:WITHDRAWAL MANAGEMENT

A supervised non-medicated withdrawal management is appropriate only for people with mild to moderate alcohol withdrawal and no known coexisting medical or psychiatric illnesses.

A supervised medicated withdrawal is required for people who are at risk of, or suffer from, alcohol withdrawal complications.

\section{STRENGTH OF RECOMMENDATIONS}

Strong

Strong

\section{SUPERVISED MEDICATED WITHDRAWAL REGIMES}

\section{All health professionals planning on prescribing or using any of the medications listed below are advised to first refer to MIMS Australia., ${ }^{\text {95] }}$}

Outlined below are the three most commonly used supervised medicated withdrawal regimes.

\section{Symptom-triggered therapy}

This regime administers medication only when the patient is symptomatic. It is suggested that this form of regime should be restricted to patients without a history of alcohol withdrawal seizures, other complications or acute concurrent medical illness, particularly as there have been no thorough studies in this area.

\section{Fixed-schedule therapy}

Benzodiazepines given at fixed dosing intervals are a common therapy for alcohol withdrawal management. For example, providing the patient is not at risk or experiencing complications, diazepam 10mg is given every six hours for two days to prevent delirium and seizures. The dose is usually tapered over the latter days of withdrawal and should not continue past day six. Additional medication can be given if required depending on health status and withdrawal symptoms. 


\section{Loading dose}

Loading dose regimes administer tailored and titrated doses of medication in the early stages of alcohol withdrawal. For example, diazepam $20 \mathrm{mg}$ is given every two hours until the alcohol withdrawal subside or the patient is sedated, reducing the chances of seizures, especially for those patients who have experienced alcohol withdrawal seizures in previous alcohol withdrawal attempts.

\section{SUMMARY OF EVIDENCE: MEDICATED WITHDRAWAL REGIMES}

Symptom-triggered therapy offers alcohol withdrawal medication as symptoms appear (not appropriate for people with a history of severe alcohol withdrawal).

Fixed schedule therapy administers withdrawal medication at set interval times over a period of days.

Loading dose regime administers tailored and titrated doses of withdrawal medication at the early stages of the alcohol withdrawal.

\section{Pharmacological interventions in withdrawal management}

\section{All health professionals planning on prescribing or using any of the medications listed below are advised to first refer to MIIMS Australia. ${ }^{95]}$}

The administration of alcohol, chlordiazepoxide, barbiturates, beta-adrenergic blockers, clonidine, acamprosate and gamma- hydroxybutyrate acid (GHB) as agents to facilitate alcohol withdrawal are not recommended and will therefore not be covered in these guidelines. Chlormethiazole is not recommended as a first line treatment and may only be useful in patients experiencing severe withdrawal symptoms. Anticonvulsant medications should not be used in routine practice, as they are not effective in preventing alcohol withdrawal complications such as seizures. However, anticonvulsants should be made available for patients currently taking them for other medical reasons. The use of major tranquillisers or anti-psychotics medications should be reserved for patients with hallucinosis and paranoid symptoms that occur during alcohol withdrawal and have not responded to a conventional sedative regime. If psychotic symptoms persist, a psychiatric evaluation may be required. All pharmacotherapy treatment listed below should be used with an alcohol withdrawal rating scale and stored in a safe environment away from patient access.

D Diazepam is a suitable medication for use in alcohol withdrawal and is considered to be the "gold standard" and first line treatment for alcohol withdrawal management. 


\section{DIAZEPAM}

Diazepam, an anti-anxiety agent or anxiolytic is widely used in medicated withdrawal from alcohol. Diazepam can be given as a loading dose, where $20 \mathrm{mg}$ is given every two hours until the withdrawal subsides or the patient is sedated after which a further dose is usually unnecessary. However, the standard therapeutic (fixed-schedule therapy) regimen involves regular doses of diazepam over two to six days. A regular starting dose is $10 \mathrm{mg}$ every six hours for two days. An additional 10mg can be supplemented if required up to a recommended two doses. The dose is usually tapered over the latter days and should not continue past day six. Outlined in Table 5.5 is the diazepam regime for ambulatory withdrawal from the New South Wales Health Department (1999) ${ }^{[97}$. Table 5.6 is an example of a diazepam regime for home-based withdrawal from Saunders et al. (1996) ${ }^{102]}$. Both tables present very similar dosing regimes.

\section{TABLE 5.5:TYPICAL DIAZEPAM REGIME FOR ALCOHOL WITHDRAWAL}

$\begin{array}{ll}\text { Day } 1 & 10 \mathrm{mg} \text { six hourly with up to } 2 \text { additional } 10 \mathrm{mg} \text { doses PRN. } \\ \text { Day } 2 & 10 \mathrm{mg} \text { six hourly with up to } 2 \text { additional } 10 \mathrm{mg} \text { doses PRN. } \\ \text { Day } 3 & 10 \mathrm{mg} \text { six hourly. } \\ \text { Day } 4 & 5 \mathrm{mg} \text { morning and night. }\end{array}$

Tapering dose may be required over the next two days.

PRN - taken as required for symptom relief.

Source: New South Wales Health Department (1999) ${ }^{[9]}$

TABLE 5.6: DIAZEPAM REGIME FOR HOME -BASED ALCOHOL WITHDRAWAL

\begin{tabular}{lllll}
\hline & $6 \mathrm{am}$ & 12 midday & $6 \mathrm{pm}$ & 12 midnight \\
\hline Day 1 & $10 \mathrm{mg}$ & $10 \mathrm{mg}$ & $10 \mathrm{mg}$ & $10 \mathrm{mg}$ \\
Day 2 & $10 \mathrm{mg}$ & $10 \mathrm{mg}$ & $10 \mathrm{mg}$ & $10 \mathrm{mg}$ \\
Day 3 & $5 \mathrm{mg}$ & $5 \mathrm{mg}$ & $5 \mathrm{mg}$ & $10 \mathrm{mg}$ \\
Day 4 & $5 \mathrm{mg}$ & $5 \mathrm{mg}$ & $5 \mathrm{mg}$ & $10 \mathrm{mg}$ \\
Day 5 & $5 \mathrm{mg}$ & - & - & $5 \mathrm{mg}$ \\
Day 6 & $5 \mathrm{mg}$ & - & - & $5 \mathrm{mg}$
\end{tabular}

*Additional 10mg dose can be given if required.

Source: Saunders et al. (1996) ${ }^{[102]}$

For patients in severe alcohol withdrawal (such as DTs) an intravenous injection of $10 \mathrm{mg}$ to $20 \mathrm{mg}$ diazepam may be given over two to five minutes. ${ }^{[1,97]}$ The patient should be observed continuously and, if necessary a further two doses given over the next hour. ${ }^{[1,9]} \mathrm{A}$ maintenance dose of one to five mg per hour (gradually reducing) by intravenous injection may be necessary over the first 24 hours or 10 to $20 \mathrm{mg}$ diazepam every two hours for six hours as required followed by 10 to $20 \mathrm{mg}$ 
every six hours until 24 hours since the initial dose. ${ }^{[1,97}$ After 24 hours the patient can usually switch to oral diazepam. ${ }^{[9]}$ The patient should be kept under close supervision.

Readers are referred to the NSW detoxification Clinical Practice Guidelines (1999) ${ }^{[9]}$ for further alcohol withdrawal diazepam treatment regimes in a specialist residential or hospital setting and for severe alcohol withdrawal.

\section{CHLORMETHIAZOLE}

Chlormethiazole is a short-acting sedative and anticonvulsant medication. Chlormethiazole is slowly being phased out and is not recommended as first line treatment. It is also not advised for outpatient withdrawal management because of its dependence-producing potential, sedative effects and lethal interaction with alcohol. ${ }^{[1,93]}$ If intravenous diazepam is unavailable or is not suitable, chlormethiazole is available in an intravenous preparation (of the edisylate salt) for patients with very severe alcohol withdrawal syndromes, such as delirium tremens, or for those on no oral intake. ${ }^{[1]}$ However, intravenous chlormethiazole carries a significant risk of respiratory depression and reduction in the gag reflex. ${ }^{[1]}$ Chlormethiazole should be taken with caution and only in fully medicated settings where the patient is closely monitored.

\section{ANTICONVULSANT MEDICATIONS}

Anticonvulsant medications such as carbamazepine have not been shown to reduce alcohol withdrawal complications such as seizures. ${ }^{1044}$ The clinical utility of these drugs seems to be outweighed by their side effects and are therefore not recommended as first line medications. The preferred course of action is to institute or increase conventional sedation using benzodiazepines such as diazepam. Anticonvulsant medications should only be made available for patients who have been taking them on a regular basis before admission and patients with epilepsy not related to withdrawal. "II If a patient suffers two or more alcohol withdrawal seizures, has focal seizures or develops status epilepticus, it should be assumed that the seizures are not a result of alcohol withdrawal and should be investigated.

\section{MAJ ORTRANQUILLISERS}

Major tranquillisers or anti-psychotic medication should only be made available to patients experiencing hallucinations where benzodiazepines are not effective. An initial dose of $2.5 \mathrm{mg}$ to $10 \mathrm{mg}$ of haloperidol orally or intramuscularly can be given if benzodiazepines, such as diazepam, are not effective in reducing or in alleviating hallucinations. Additional doses of haloperidol can be given if the patient continues to experience hallucinations. ${ }^{[1,97,105]}$ The patient should also be monitored carefully for hypotension. 


\section{VITAMINS}

All patients in alcohol withdrawal and all alcohol-dependent patients should receive thiamine as a preventive measure against Wernicke-Korsakoff's syndrome. An intramuscular dose of at least $100 \mathrm{mg}$ of thiamine should be given each day for three days. ${ }^{[1,94,97,106]}$ If intramuscular administration is not possible, $100 \mathrm{mg}$ of thiamine should be given orally three times a day for at least a week. ${ }^{[1,}$ ${ }^{94]} \mathrm{A}$ large dose such as this is necessary because oral thiamine is poorly and erratically absorbed in alcohol-dependent patients. Thiamine should be given before any glucose infusion, since a glucose load may further deplete thiamine and precipitate the onset of Wernicke-Korsakoff's syndrome. ${ }^{[1,97]}$ Deficiencies of other B complex vitamins and of vitamin $\mathrm{C}$ are not uncommon and an oral multi-vitamin preparation should be given for a few days.

\section{FLUIDS AND ELECTROLYTES}

The fluid status of patients must be carefully assessed and when necessary intravenous saline or dextrose-saline should be administered. Dextrose solutions should not be given until the patient has received at least one dose of thiamine 100mg parenterally, as a glucose load in a patient with borderline thiamine status is likely to precipitate Wernicke-Korsakoff's syndrome. ${ }^{[1,97,106]}$ Low levels of potassium and magnesium are common in severe withdrawal and may lead to neuromuscular problems. Potassium and magnesium supplements can be administered, however, no evidence exists to support the use of magnesium alone or with benzodiazepines for alcohol withdrawal. Patients should be treated with the appropriate supplements of potassium ( 80 to $240 \mathrm{mmol}$ per day) or magnesium (40 to $100 \mathrm{mmol}$ per day). ${ }^{[1,97]}$

\section{SUMMARY}

A number of medications have a place in the management of severe alcohol withdrawal:

- Diazepam is recommended as "gold standard" and as first-line treatment because of its relatively long half-life and evidence for effectiveness for a number of reasons.

- Chlordiazepoxide, barbiturates, beta-adrenergic blockers, clonidine acamprosate and GHB, have no routine place in the management of the alcohol withdrawal syndrome given the effectiveness of other treatments.

- Chlormethiazole should be taken with caution and only in fully supervised medicated withdrawal setting where the patient is closely monitored.

- Major tranquillisers or anti-psychotic medications such as haloperidol should only be used for alcohol withdrawal complications such as hallucinations when diazepam is not effective.

- Intravenous chlormethiazole should be used only in severe alcohol withdrawal situations when intravenous diazepam is not available or is not suitable.

- Anticonvulsant medications should be made available for patients who have taken them on a regular basis and for patients with epilepsy not related to withdrawal.

- Electrolyte replacement may be a necessary adjunctive treatment for some patients.

- All patients who are experiencing the alcohol withdrawal syndrome should receive thiamine and an oral multi-vitamin preparation each day for a week. 
Diazepam is the "gold-standard" and first-line treatment for alcohol withdrawal managemen.

Barbiturates, major tranquillisers or anti-psychotic medications, beta-adrenergic blockers, chlormethiazole, clonidine and chlordiazepoxide, have no routine place in the management of the alcohol withdrawal syndrome.

Anticonvulsants are not recommended as first-line treatment for alcohol withdrawal, however should be given to patients who take them on a regular basis before admission and to patients with epilepsy not related to alcohol withdrawal.

However, major tranquillisers or anti-psychotic medication such as haloperidol should be made available for patients experiencing hallucinations when diazepam is not effective in reducing or alleviating hallucinations.

$100 \mathrm{mg}$ of intramuscular or oral thiamine should be administered to any patient at risk of alcohol withdrawal. Thiamine needs to be given before any glucose intake to prevent Wernicke-Korsakoff's syndrome.

Multi-vitamins should be given during the alcohol withdrawal phase.

Patients in alcohol withdrawal should have their fluid status IV monitored regularly, fluids given, and if needed, intravenous saline or dextrose-saline should be considered (only after 100mg thiamine has been administered).

\section{RECOMMENDATIONS: PHARMACOLOGICALWITHDRAWAL MANAGEMENT}

Diazepam is recommended as the "gold-standard" and first-line treatment for alcohol withdrawal symptoms.

Anticonvulsant medications are recommended for use with patients who take them on a regular basis before admission and with patients who have epilepsy not related to alcohol withdrawal.

Haloperidol (a major tranquilliser or anti-psychotic medication) is Strong recommended for use with patients experiencing hallucinations and/or paranoid symptoms where diazepam is not effective in reducing or alleviating the hallucinations and paranoid symptoms.

$100 \mathrm{mg}$ of intramuscular or oral thiamine should be administered before glucose to any patient at risk of alcohol withdrawal.

Moderate

Multi-vitamins and fluids should be given to any patient withdrawal from alcohol. 
Chapter 6: Post-withdrawal treatment setting 


\section{Chapter 6: Post-withdrawal treatment setting}

Given the range of needs and circumstances among alcohol dependent patients, it makes sense to have different treatment options to meet these needs. The evidence reviewed for these guidelines suggests that there is a valid role for the full spectrum of treatments, from brief interventions through to intensive inpatient or residential rehabilitation. However, the evidence does not support residential rehabilitation for most patients as most seem to do equally well in non-residential settings.

\section{CHAPTER AIM}

The aim of this chapter is to:

- Describe the range of post-withdrawal treatment settings available in Australia.

- Present evidence about matching patients to treatment setting.

The 1993 guidelines ${ }^{[1]}$ highlighted the debate about the relative efficacy of residential and nonresidential treatment settings. Since then, the emphasis has shifted towards identifying those who benefit most from residential treatment, and those who are better treated on a non-residential basis. Residential treatment is a less cost-effective option if it is used by those who would benefit equally well from less intensive, less expensive interventions. There is no evidence of a need for residential intervention for those who have mild to moderate levels of dependence, and some patients with low alcohol involvement may fare worse in a residential setting. ${ }^{[107]}$

Nevertheless, it seems that residential treatment does confer specific benefits for some patients.

\section{The benefits are most evident for those with impaired cognitive functioning, serious psychiatric disorders that may affect their progress, those who have failed repeatedlly with non-residential care, or those who are in a socially unstable environment that might affect their success with non-residential care. ${ }^{[87,107]}$}

There is also some evidence that suggests that residential treatments differ in their effectiveness $[108,109]$, and it may be that the more effective residential treatments might be superior to a nonresidential intervention ${ }^{3}$. However, as discussed in the 1993 guidelines there is insufficient evidence to resolve this issue.

\section{KINDS OF RESIDENTIAL CARE}

A distinction needs to be made between standard residential care, and the therapeutic community (TC) approach. In the latter case, therapeutic communities emphasise a holistic approach to treatment, which implies addressing the psychological and other issues behind substance abuse. The community is viewed as both the context and method in the treatment process. In the former, residential programs use similar treatment approaches to those seen in non-residential services, albeit more intensively.

3 The argument could be mounted in the reverse fashion. That is, outpatient interventions might be improved by careful attention to the nature and appropriateness of the procedures used. 
TCs are a complex mix of elements that can be difficult to define. However, the Australasian Therapeutic Communities Association (ATCA) Better Practice Project identified the following features of therapeutic communities: ${ }^{[110]}$

1. "Residents participate in the management and operation of the community.

2. The community, through self-help and mutual support, is the principal means for promoting behavioural change.

3. There is a focus on social, psychological and behavioural dimensions of substance use". (p. 10)

\section{Who should receive residential treatment?}

Matching clients to treatment setting remains a clinical decision, however Melnick's (2001) Client Matching Protocol (CMP) may be helpful in determining who should be referred to residential or non-residential therapeutic community settings. ${ }^{[111]}$ To date, only one study has evaluated the CMP, which consists of a 30-item individually administered questionnaire and a decision tree. The questionnaire is currently not available, but the decision points are outlined below:

1. Individuals with a low risk pattern of drug use are referred to non-residential treatment. Highrisk individuals enter into the second assessment point.

2. Individuals with one year or more of abstinence in the last four, or a drug history of less than four years, are referred to non-residential treatment. Those not meeting this criterion move to the third point.

3. If social factors (drug-free domicile, peer involvement with drugs, criminal behaviour) are scored as high-risk, residential treatment is recommended. Others are referred to the final assessment point.

4. Individuals who are not habilitated (do not have a high school diploma, technical training or sufficient work skills and experience to earn a living) are referred to residential treatment. Others are referred to non-residential treatment. ${ }^{[11]}$

Residential care should be considered in a number of circumstances set out below.

- A residential intervention may be a suitable alternative option for chronic relapsing cases when non-residential interventions have been unsuccessful. Indeed, residential services might be particularly valuable for this chronic subgroup of drinkers who relapse and incur severe damage to their health.

- Residential care should be considered for those clients with severe dependence on alcohol coming from an environment that will not support changes in drinking behaviour.

- For those with a high level of physical dependence on alcohol, who are most likely to require medicalised care, including medical detoxification, brief residential care may be suitable. This decision depends on the physical status of the patient as assessed by suitably qualified practitioners, and the likelihood of an imminent severe withdrawal syndrome. Please refer to Chapter 5: Withdrawal management for more information.

- Residential care should be considered where there are concurrent psychiatric complications such as alcoholic hallucinosis, depression and psychotic states, especially where there is reason to believe that these disorders will affect withdrawal management. 
- A residential intervention can be particularly useful for those patients with moderate to severe alcohol-related brain damage such as Wernicke-Korsakoff's Syndrome and other alcohol amnestic disorders that may affect the person's ability to cope with, or to even attend regularly, a non-residential intervention. ${ }^{[8]}$ Not all such people require a residential program. However, evidence of organic brain damage, plus an inability to live independently, a lack of support in the community, and a likelihood of sudden relapse to heavy drinking need to be weighed in the decision to admit a person with alcohol-related brain damage to a suitable program. These patients are likely to benefit from a structured intervention within a residential setting that includes:

$\triangleright$ The opportunity to detoxify from alcohol

$\triangleright \quad$ A clear daily routine

$\triangleright \quad$ Presentation of therapeutic messages to decrease alcohol consumption using a simple and repetitive approach

$\triangleright \quad$ Participation in training aimed at helping them to deal with their memory problems, such as notebooks, diaries, and other mnemonic devices and skill [112]

\section{$\triangleright \quad$ Practice in non-drinking social activities}

The content of a residential program that might best suit these patients is exemplified by, but is not necessarily unique to those that use a 12-step approach. ${ }^{[113]}$ This is not a general endorsement of the 12-step approach to treatment without the addition of other interventions.

- Residential care for the homeless and for the severely deteriorated, malnourished or socially unstable alcohol dependent persons is required for therapeutic and humanitarian reasons. While providing shelter and welfare services is not specifically treatment for alcohol dependence, for severely affected and dependent patients the stability provided by residential care it is a necessary prerequisite to treatment. Providing these services and general health care must be continued for those who need it.

Where residential intervention occurs, there is a further distinction to be made between the therapeutic potential of the settings to produce changes in alcohol intake, and the welfare functions that some of these facilities fulfil. The welfare functions fulfilled by some alcohol and drug residential facilities include the provision of beds and shelter for those who need them, and providing a place where the drinker can be away from drinking alcohol for a period, even though there could be a retum to drinking after leaving the facility. For some more chronically dependent patients, residential facilities offer a place to belong, a sense of community and a safe and structured way to live in times of need and crisis. Although the responsibility for the welfare needs of such people might properly belong to another area of community service, for many patients this need may currently only be provided by alcohol and drug facilities.

Residential programs that intervene to alter drinking should include a full assessment of the patient. They should offer a range of treatment modalities that can be tailored to the needs of the presenting problem. They should have clearly articulated aims and objectives, and they should have an evaluation component in place. They should also have a number of relapse prevention strategies to support those who might relapse upon return to their normal environment. 


\section{Potential improvements to residential/therapeutic community care}

The following comments are tentative, given the limited evidence to support them. No doubt, future phases of ATCA Better Practice Project will provide more substantial recommendations than can be made here. ${ }^{[101}$

Given the consistent finding that motivation mediates residential treatment outcomes, using motivational interviewing as preparation for treatment may improve outcomes. ${ }^{[3]}$ Motivational intervention appears to exert its influence on treatment outcome by increasing treatment participation.

An important issue for all treatment services, including residential care, is the need to improve retention. Structured and interactive information sessions conducted by experienced staff members at the start of therapeutic community treatment appear to improve retention in treatment for clients who lack motivation to change. Seminar themes reflected the therapeutic community approach to treatment and recovery, for example self-esteem, self-help and recovery concepts, therapeutic community philosophy and expectations, therapeutic community retention and success rates. Problems of staying in treatment and client concerns were addressed. It has been suggested that motivated clients are more likely to have higher therapeutic involvement, resulting in initial improvement and in sustaining motivation for remaining and participating in treatment. ${ }^{[114]}$ For more detail on treatment retention, refer to Chapter 10: Extended care.

Finally, tailoring therapeutic communities to clients' needs, specifically those associated with psychiatric comorbidity and cognitive deficits, may produce better outcomes across a range of areas. In a study conducted with a group of homeless, chemically dependent, psychiatrically disordered males ${ }^{[109]}$, changes made to improve treatment outcomes included:

- Administration of psychiatric medications

- Increased program flexibility

- Less confrontation

- Increased psycho-educational instruction

- Fewer sanctions

- More explicit affirmation for achievements

- Greater responsiveness to individual needs

- Greater freedom to come and go from the residential facility early in treatment

- Allowing clients to leave the residence to attend a day treatment program for mentally ill, chemically dependent individuals

- Reduced therapeutic community duties

- Staff provided more assistance to clients in running program interventions and directing client activities

- The program was structured to have fewer activities and shorter interactions

The above research is US-based. This modified approach is already offered by many therapeutic communities in Australia, including shorter planned stays which appear to be a viable option for many clients. 
The evidence on non-residential versus residential treatment

III-1 setting is equivocal. Variables such as client motivation and treatment modality appear to have more effect on treatment outcome than treatment setting.

Clients high in alcohol dependence may benefit more from residential than non-residential care; those with low alcohol dependence may fare better in non-residential settings.

Clients low in cognitive functioning may benefit more from residential than non-residential care, although this finding appears to be moderated by AA attendance.

In some circumstances, the availability of inpatient/residential III-2 treatment is indicated, such as for chronic relapsers, those with significant comorbid mental disorders, an unstable social environment, severe cognitive impairment, medical complications requiring residential care, or homeless clients.

Retention in treatment may be improved by conducting structured, interactive induction sessions looking at the problems of staying in residential treatment and other client concerns.

Residential treatment programs modified to meet the needs III-1 of mentally ill, homeless clients appear to be more effective with this population than residential "treatment as usual".

Strategies to increase treatment adherence, for example, motivational interviewing at the start of residential treatment may improve treatment outcomes.

Decisions about treatment setting should be based on the Strong client's treatment goals, preferences, severity of dependence, the presence of comorbid disorders, cognitive and social functioning, relapse history, and social circumstances.

Residential programs should aim to increase retention in treatment by conducting structured, interactive induction sessions looking at the problems of staying in treatment and other client concerns, by using motivational interviewing techniques, and by strengthening the client's involvement in treatment and therapeutic alliances.

Residential programs should be modified to meet the needs of Moderate particular groups, for instance those with comorbid disorders, those with childcare responsibilities, and the homeless. 
Chapter 7: Brief interventions 


\section{Chapter 7: Brief interventions}

\section{CHAPTER AIM}

The aim of this chapter is to provide information about:

- The common elements of brief interventions.

- To whom brief interventions should be delivered.

- In what settings brief interventions should be delivered.

- Who should deliver these interventions.

There is strong evidence to support the effectiveness of brief interventions for the treatment of alcohol problems. ${ }^{[115,116]}$ When implemented in primary care settings, brief interventions are aimed towards risky drinkers who do not currently suffer from alcohol use disorders. Brief interventions are more effective than no intervention in reducing consumption in risky drinkers who have not sought treatment for alcohol problems. ${ }^{[115]}$ Among patients who have sought treatment for alcohol use problems, there is no evidence indicating that brief interventions are any less effective than more intensive treatments. ${ }^{[115]}$ The interventions offered to non-treatment seeking populations are typically different from the interventions offered to treatment seeking clients. ${ }^{[117]}$

Brief interventions are now recognised as an important part of the overall approach to dealing with risky drinking in Australia. Individuals targeted by brief interventions are not only those people who have severe alcohol problems but also those who have a pattern of consumption that is defined as at-risk for health, although they may not currently have any alcohol-related problems. The aim of brief interventions is to reduce the prevalence of alcohol abuse and dependence by detecting and intervening with risky drinkers before they progress to alcohol abuse and dependence.

\section{WHAT IS A BRIEF INTERVENTION?}

Brief interventions generally range from 5 to 30 minutes duration, although some are more extensive, consisting of several sessions delivered over several months. The procedures used in the delivery of brief interventions often involve a combination of motivational interviewing and counselling. There are two broad classes of brief interventions: opportunistic interventions offered to patients detected as drinking beyond recommended levels as defined by the Australian Alcohol Guidelines ${ }^{[34}$. These interventions are offered to patients who have not sought treatment and are often offered in primary care settings. The other form of brief interventions are those offered instead of more intensive interventions, usually to treatment-seeking people who often have more serious alcohol problems.

Opportunistic brief interventions are aimed at risky rather than dependent drinkers. The aim is to inform patients that they are drinking at levels that could lead to health problems, and to encourage them to decrease consumption so as to reduce the risk of future health problems. Targets for opportunistic brief interventions are usually identified through screening. The AUDIT, discussed further Chapter 3: Screening and assessment, is often used in primary care settings as a screening tool.

Although there are a variety of treatment interventions, which claim to be brief interventions, the 
acronym FRAMES (Table 7.1) provides an outline of the important components of a brief intervention. As a general rule, brief interventions should include these six components.

\section{TABLE 7.1: COMMON ELEMENTS OF A BRIEF INTERVENTION}

\section{Common elements of a brief intervention}

Feedlback Personal Feedlback about the risks associated with continued drinking, based on current drinking pattems, problem indicators, and health status.

Responsibility Emphasis on the individual's personal Responsibility and choice to reduce drinking behaviour.

Advice Clear Advice about the importance of changing cument drinking pattems.

Menu

A Menu of altemative change options. This emphasises the individual's choice to reduce drinking patterns and allows them to choose the approach best suited to their own situation. Altematives include setting a specific limit on alcohol consumption, leaming to recognise the antecedents of drinking and developing skills to avoid drinking in high-risk situations, pacing one's drinking and leaming to cope with everyday problems that lead to drinking.

Empathy Empathy from the person providing the intervention is an important determinant of patient motivation and change. A warm, reflective and understanding brief intervention is more effective than an aggressive, confrontational or coercive style.

Self-efficacy Self-efficacy involves instilling optimism in the patient that his or her chosen goals can be achieved. It is in this step, in particular, that motivation-enhancing techniques are used to encourage patients to develop, implement and commit to plans to stop drinking.

Source: Bien, Miller and Tonigan (1993). Reproduced with permission. ${ }^{[16]}$

A typical five to ten minute brief intervention might involve advice on reducing consumption in a persuasive but non-judgemental way. Personalised information contains an assessment and discussion of the patient's consumption level and how it relates to general population consumption. The clinician should discuss the potential health problems that can arise from risky alcohol use and help the patient set goals for changing patterns of consumption. Advice can be supported by self-help materials which provide information about the potential harms of risky alcohol consumption and can provide additional motivation to change.

Brief interventions are usually motivational. Although some patients who are identified as drinking at risky levels do not perceive change as necessary, providing them with advice and information about the potential consequences of continued use may help them recognise that they may have a problem. Other patients may acknowledge that they are drinking too much and be aware that risky alcohol use can be harmful. Brief intervention can be particularly successful for this group of patients, as the clinician provides encouragement and support for these clients. 


\section{WHO SHOULD BRIEF INTERVENTIONS BETARGETED AT?}

Opportunistic brief interventions are generally targeted to people who are drinking beyond recommended limits, but may not yet be experiencing health problems. The aim of brief interventions is to identify risky drinkers and modify their behaviour to prevent them from progressing to alcohol abuse and dependence.

If a patient returns to the setting in which he or she was delivered a brief intervention, and is still drinking to excess, the clinician should suggest to the patient that they may benefit from a more intensive treatment program in an effort to reduce levels of drinking, and refer the patient to a specialist alcohol and drug treatment service.

\section{WHERE SHOULD BRIEF INTERVENTIONS BE DELIVERED?}

Opportunistic brief interventions can be delivered in a variety of settings including general practice settings, general hospital wards, emergency hospital wards and community counselling centres.

\section{General practice settings}

General practitioner (GP) settings are a good setting in which to deliver brief interventions as about 85 percent of the population visit their general practitioner each year [118], allowing for the detection of a large proportion of risky drinkers. Further, current data suggests that about 25 percent of patients presenting to general practitioner settings in Australia are likely to be drinking at risk levels. ${ }^{[1199}$ Further, GPs have the resources and skills to offer an intervention. Thus, the GP has the potential to have a substantial effect on risky levels of drinking.

\section{General hospital wards}

Clear associations have been found between admissions for traumatic incidents or medical problems and alcohol consumption. ${ }^{[120]}$ There is a high prevalence of problem drinkers among hospital patients, making general hospital wards a good environment in which to offer brief interventions to a large number of risky drinkers who show, or may be at risk of developing alcohol problems. ${ }^{[1211}$ Hospital wards can be a particularly effective setting for brief interventions, as patients appear to be more motivated and willing to change their drinking behaviours after being hospitalised. ${ }^{[122]}$

\section{Emergency departments}

Many people attending accident and emergency departments have alcohol-related injuries. Emergency wards are likely to be particularly effective settings for brief interventions, as patients have usually through a traumatic experience, and preliminary data suggest that a recent lifethreatening experience increases the receptivity of patients to interventions, increasing the likelihood of brief interventions being effective in reducing alcohol consumption among these patients. ${ }^{[122-126]}$

\section{Community counselling services}

Patients may present to community counselling services with a variety of complaints. Some of these patients may be presenting with an alcohol or other drug problem, and others may have completely unrelated concerns. Whether patients are presenting with a primary alcohol problem, or another complaint, patients who are drinking alcohol at risky levels should be given a brief intervention. 


\section{Workplace settings}

Rates of risky alcohol consumption are particularly high in some workplace settings. In particular, Australia Post employees and police have been identified as having a large proportion of risky drinkers. ${ }^{[127,128]}$ Brief interventions for risky alcohol consumption have been embedded within the context of a broader health promotion campaign and delivered in workplace settings. ${ }^{[12,}{ }^{129]}$ Workplace settings where there are high levels of risky alcohol consumption can be effective locations for the delivery of brief interventions.

\section{WHO CAN DELIVER BRIEF INTERVENTIONS?}

Any health professional or treatment provider can deliver brief interventions, provided they have been trained in the provision of brief interventions.

\section{BARRIERSTO IMPLEM ENTING BRIEF INTERVENTIONS}

In spite of evidence supporting the effectiveness of brief interventions, their delivery has not been integrated into general practice settings, general or emergency hospital wards or community counselling services.

Barriers to implementing brief interventions include:

- Practitioner's lack of knowledge and skills

- Lack of time

- Lack of financial incentives

- Organisation of the health care system

- Lack of professional reward

- Lack of diagnostic aids for alcohol-related problems. ${ }^{[13]}$

\section{SUMMARY}

- Brief interventions are typically of five to 30 minutes duration.

- Brief interventions involve motivational interviewing and counselling techniques.

- The aim of brief interventions is to alert the drinker that they are drinking at levels that could lead to health problems, and encourage them to reduce their consumption to reduce the risk of future health problems.

- The acronym FRAMES provides an outline of the important components of a brief intervention.

- Brief interventions are usually targeted at individuals who are risky rather than dependent drinkers.

- Brief interventions can be provided in a number of settings including general practitioner settings, general and emergency hospital wards, community counselling centres and workplace settings.

- Any health care professional or treatment provider can deliver brief interventions, as long as that person has training in the provision of brief interventions. 


\begin{tabular}{l|l|l}
\hline SUMMARY OF EVIDENCE: BRIEF INTERVENTIONS & LEVEL OF EVIDENCE \\
$\begin{array}{l}\text { Brief interventions are effective in reducing risky alcohol } \\
\text { consumption among non-treatment seeking patients } \\
\text { who drink excessively. }\end{array}$ & I \\
There is no evidence indicating that brief treatments are \\
less effective than more intense interventions in \\
treatment-seeking populations. \\
$\begin{array}{l}\text { Patients who have recently suffered trauma are usually } \\
\text { more receptive to treatment for alcohol problems and } \\
\text { are therefore ideal patients for brief interventions. }\end{array}$ \\
$\begin{array}{l}\text { RECOMmENDATIONS: BRIEF INTERVENTIONS } \\
\text { Brief interventions should be implemented into routine } \\
\text { practice in general practice settings, general and } \\
\text { emergency hospital wards, and community } \\
\text { counselling centres. }\end{array}$ \\
$\begin{array}{l}\text { Brief interventions should consist of the six components } \\
\text { of the FRAMES acronym: feedback, responsibility, advice, } \\
\text { menu, empathy and self-efficacy. }\end{array}$ \\
$\begin{array}{l}\text { Where brief interventions are not successful in reducing } \\
\text { alcohol consumption, a more intensive treatment should } \\
\text { be offered to the client. }\end{array}$ \\
LEVEL OF RECOMIMENDATION \\
\hline Strong \\
\hline
\end{tabular}


Chapter 8: Psychosocial interventions 


\section{Chapter 8: Psychosocial interventions}

\section{CHAPTER AIM}

The aim of this chapter is to provide health care workers with knowledge and guidance about:

- the principles and goals of general counselling and specific psychosocial interventions

- general issues to do with psychosocial interventions, including the effect of clinicians on treatment outcome, and of the importance of using standardised treatments.

\section{Characteristics of effective clinicians}

The effect of clinician characteristics on treatment for alcohol use disorders has received far less attention than therapy and client variables. The difficulty of studying clinician effects has contributed to this lack of investigation. ${ }^{[130]}$ However, there is some evidence on clinician effects which suggests that the effect of therapy is mediated by clinician characteristics. Having strong interpersonal skills has been most strongly and consistently associated with higher treatment effectiveness. ${ }^{1311,132]}$ Najavits et al. ${ }^{[132,133]}$ found a marked effect of differing clinician style on the outcome of behavioural interventions. Clients of clinicians who were more interpersonally skilled, less confrontational or more empathetic were found to have better outcomes. ${ }^{1132]}$ Differences in clinicians' effectiveness were not attributable to training, treatment orientation or experience. ${ }^{[134]}$ When a supportive style is compared with a confrontational style, confrontation is associated with increased client resistance and higher levels of drinking. ${ }^{134]}$ In fact, the more clinicians confronted clients, the more clients drank. An Australian study reported in 2002 that better treatment outcome was associated with client's perceptions of clinician expertise and empathy. ${ }^{[3]}$

Competence of clinicians is also likely to be related to treatment outcome. A study by Broome et al. (1999) ${ }^{[135]}$ looked at clinician competence based on clients' ratings of organisation, self-confidence, helpfulness and knowledge. Perceived competence was significantly related to re-arrest rates of the 279 clients, accounting for 42 percent of variance.

Clinician effects are also related to adherence to treatment manuals (discussed later in this chapter). According to a meta-analysis which included, but was not limited to, substance use disorder outcome studies, clinician effects on treatment outcome decrease when adhering to treatment increases. ${ }^{[136]}$

Together, these findings suggest that clinicians who can form a warm, supportive relationship with the client, and who can show empathy, are likely to achieve greater improvement in client functioning. It also suggests that clinicians develop an organised approach to client management, keeping careful case and progress notes, which helps in anticipating difficulties and makes planning and practising methods to deal with difficult situations before they arise easier.

\section{SUMMARY}

Clinicians should be trained in skills that enable them to:

- show empathy

- develop a supportive relationship with the client

- have an organised approach to each case. 
The impact of treatment interventions are partly

mediated by clinician effects.

Interpersonal skills are the most studied clinician characteristic, and clinicians with strong interpersonal skills are more effective.

Competent, empathetic clinicians achieve better

III-1 treatment outcomes for clients

\section{RECOMMENDATIONS: CLINICIAN CHARACTERISTICS}

\section{STRENGTH OF RECOMMENDATION}

Clinicians should receive interpersonal skills training which focuses particularly on empathy.

Clinicians should have an organised approach to treatment, and take careful case and progress notes.

\section{Counselling}

General counselling and associated skills are effective for counselling people who have problems with alcohol. Counselling skills such as listening and empathy should form the basis of any therapeutic relationship. However, counselling is not usually sufficient to change drinking behaviours and should be supported by more specific techniques.

The basic counselling skills advocated by "the skilled helper" model are recommended for developing rapport with clients, and for dealing with distress and ambivalence about receiving treatment and about the goals of treatment. ${ }^{[137]}$ The two principal goals of counselling are:

1. To help clients manage their problems in order to live more effectively, and to develop unused resources and missed opportunities more fully.

2. To help clients become better at helping themselves in their everyday lives.

Egan (2002) ${ }^{[137]}$ has developed a model which provides a general framework for the counselling process, and covers all aspects of dealing with the client, except for specific treatment interventions. According to Egan, all worthwhile helping frameworks help clients ask and answer for themselves, four fundamental questions:

1. 'What's going on?"

What are the problems, issues, concerns, or opportunities I should be working on? This stage involves helping clients clarify the key issues calling for change.

2. "What solutions make sense for me?"

What do I want my life to be like? What changes would make me happier? This stage involves helping clients identify anticipated outcomes from treatment.

3. "How do I get what I need or want?'

What plan will get me where I want to go? This stage involves helping clients develop strategies for accomplishing goals.

4. “How do I get results?" 
How do I turn planning and goal setting into solutions, results, outcomes or accomplishments? How do I get going and keep going? This stage represents the implementation stage of the model.

These four questions, turned into three "stages" and an action phase, provide the basic framework for the helping process (Table 8.1). There are three steps within each stage, and the goal of each stage is to help the client move towards better management of problems and taking advantage of opportunities. The three stages overlap and interact with one another.

\section{TABLE 8.1: STAGES AND STEPS OFTHE SKILLED HELPER MODEL}

\section{STAGE I: WHAT'S GOING ON?}

Step I-A: Help the clients tell their stories.

Step I-B: Help clients break through blind spots that prevent them from seeing themselves, their problem situations, and their unexplored opportunities as they really are.

Step I-C: Help clients choose the right problems and/or opportunities to work on.

\section{STAGE III:WHAT SOLUTIONS MAKE SENSE FOR ME?}

Step III-A: Help clients use their imaginations to spell out possibility for change.

Step II-B: Help clients choose realistic and challenging goals that are real solutions to the key problems and unexplored opportunities identified in Stage I.

Step III-C: Help clients find the incentives to help them commit themselves to their change agendas.

\section{STAGE III: WHAT DO I HAVETO DOTO GET WHAT I NEED OR WANT?}

Step IIII-A: Possible actions: Help clients see that there are many different ways of achieving goals.

Step IIII-B: Help clients choose best-fit strategies.

Step III-C: Help clients craft a plan.

Source: The Skilled Helper. Reproduced with permission. ${ }^{[137]}$

Figure 8.1 represents the basic framework of the skilled helper model. For more detail about the model, see The Skilled Helper: A problem-management and opportunity-development approach to helping. [137] 
FIGURE 8.1:THE SKILLED HELPER MODEL

Stage 1:

“What's going on?"

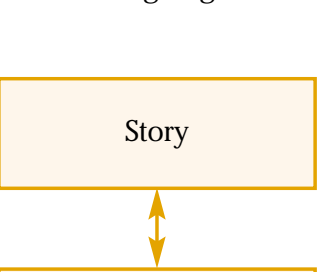

Stage 2:

"What solutions make sense for me?"

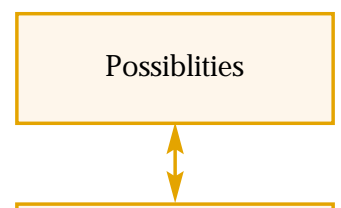

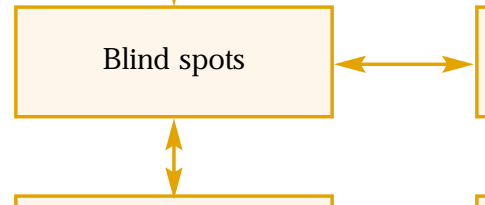

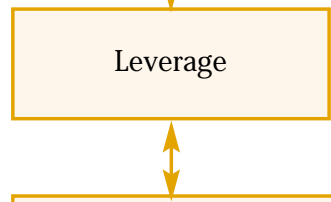

Change agenda
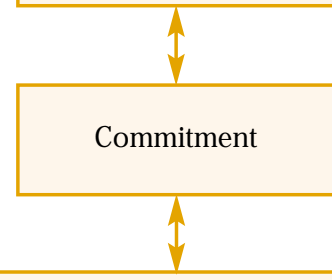

How do I make it all happen?

Source: Egan (2002). Reproduced with permission. ${ }^{[137]}$

While the model provides a general framework for the counselling process, effective communication is also viewed as a key element of good counselling. The basic communication skills for effective counselling are:

- Visibly tuning in to the client, which refers to the clinician giving their complete attention to the client.

- Active listening, in which the clinician focuses on understanding what their client is saying, both directly and indirectly.

- Checking that there is a shared understanding between clinician and client of what is being communicated. ${ }^{[137]}$

The arrow of the model represents the action phase of the model. While the nine steps of the model revolve around planning for change, action should be incorporated into the counselling process. The action stage of the process will often incorporate more specific treatment interventions that help the client deal with specific problems or deficits.

As noted above, it is not expected that counselling alone is sufficient to change the drinking behaviour of most clients. Rather, the goal of counselling is to develop a relationship between the clinician and the client, which supports implementing specific strategies designed to combat the drinking problem. 


\section{The counselling process aims to:}

- Build a trusting relationship in which the client and the clinician cooperate in planning and implementing the intervention. This mutual activity provides a supportive situation in which the client can actively work towards change.

- Reduce the client's fear and distrust of treatment programs and thereby encourage the client to continue attending treatment and follow-up appointments.

- Provide a non-threatening and supportive environment in which the client can address sensitive issues, ${ }^{[1]}$

\section{SUMMARY}

Supportive and empathic counselling skills are recommended as the basis from which to deliver appropriate treatment interventions that may be required to deal with problems and deficits that predispose the client to risky drinking.

\begin{tabular}{l|c}
\hline RECOMIMENDATIONS: COUNSELLING & STRENGTH OF RECOMIMENDATION \\
$\begin{array}{l}\text { General counselling skills should form the basis } \\
\text { of a therapeutic relationship. }\end{array}$ & Strong \\
$\begin{array}{l}\text { General counselling skills should be supported } \\
\text { by more specific techniques aimed at initiating } \\
\text { a change in behaviour. }\end{array}$ & Strong \\
\hline
\end{tabular}

\section{Motivational interviewing}

Motivational interviewing is a procedure which was introduced by Miller and Rollnick in 1991. ${ }^{[138]}$ They define motivational interviewing as "a client-centred, directive method for enhancing intrinsic motivation to change by exploring and resolving ambivalence". ${ }^{1399}$ The goal of motivational interviewing is to steer the client towards motivation for change by eliciting reasons for change from the client. Motivational interviewing is client-centred, emphasising that behaviour change is voluntary, and that responsibility for decisions and results of behaviour change rests with the client. It is directive, aimed toward a resolution of ambivalence.

Motivational interviewing is viewed as a method of communication rather than a set of techniques, or a specific strategy. Motivational interviewing can be delivered as a prelude to treatment and as a standalone treatment, or can be integrated with other treatment interventions. There is reasonable evidence supporting the effectiveness of motivational interviewing as an effective stand-alone treatment for risky and dependent drinkers. The evidence on motivational interviewing as a prelude to treatment is equivocal.

Miller and Rollnick suggest three general processes through which motivational interviewing may achieve its effects, namely, collaboration, evocation and autonomy (Table 8.1). ${ }^{[1399} \mathrm{A}$ collaborative relationship between the clinician and the client is a key element of motivational interviewing. The counsellor should communicate a partner-like relationship, providing support rather than persuasion or coercion. Evocation refers to the role of the counsellor to elicit resources and motivation for change from within the client. Autonomy relates to the client's independence and responsibility for change, which must be affirmed and supported by the counsellor. 


\section{TABLE 8.2:THE SPIRIT OF MOTIVATIONAL INTERVIEWING}

\section{Fundamental approach of motivational interviewing}

Collaboration. Counselling involves a partnership that honours the client's experiences and perspectives. The counsellor provides an atmosphere that is conducive rather than coercive to change

Evocation. The resources and motivation for change are presumed to reside within the client. Intrinsic motivation for change is enhanced by drawing on the client's own perceptions, goals and values.

Autonomy. The counsellor affirms the client's right and capacity for self-direction and facilitates informed choice.

\section{Mirror-image opposite approach to counselling}

Confrontation. Counselling involves over-riding the client's impaired perspectives by imposing awareness and acceptance of "reality" that the client cannot see or will not admit.

Education. The client is presumed to lack key knowledge, insight, and/or skills that are necessary for change to occur. The counsellor seeks to address these deficits by providing the requisite enlightenment.

Authority. The counsellor tells the client what he or she must do.

Source: Miller and Rollnick, 2002. Reproduced with permission. ${ }^{[139]}$

There are four broad, guiding principles that underlie motivational interviewing: express empathy, develop discrepancy, roll with resistance and support self-efficacy (Table 8.2). An empathetic counselling style is a fundamental and defining characteristic of motivational interviewing. Empathy is seen as the foundation on which motivational interviewing is built. The expression of empathy refers to an attitude of acceptance and respect for the drinker, and involves reflecting the individual drinker's concerns about risky alcohol consumption. A second general principle of motivational interviewing is to create a discrepancy between the client's present behaviour and his or her broader goals and values. The notion of developing discrepancy is aimed at clarifying important life goals with the drinker, and exploring the consequences of continued drinking that conflict with those goals. The technique of rolling with resistance is related to avoiding argumentation, as argumentation is counterproductive. Rather, resistance of a client can be reframed to create momentum towards change. The last principle of motivational interviewing involves the concept of self-efficacy which refers to a person's belief in his or her ability to carry out and succeed with a specific task. 


\section{TABLE 8.3:THE GUIDING PRINCIPLES OF MOTIVATIONAL INTERVIEWING}

\begin{tabular}{|ll}
\hline Principle 1: & - Acceptance facilitates change \\
\hline Express empathy & - Skilful reflective listening is fundamental \\
& Ambivalence is normal \\
\hline Principle 2: & - The client should present arguments for change \\
\hline Develop discrepancy & - Change is motivated by a perceived discrepancy between present \\
& behaviour and important personal goals or values \\
\hline Principle 3: & - Avoid arguing for change \\
\hline Roll with resistance & - Do not directly oppose resistance \\
\hline Principle 4: & - New perspectives are invited but not imposed \\
\hline Support self-efficacy & - The client is a primary resource in finding answers and solutions \\
\hline
\end{tabular}

Source: Miller and Rollnick, 2002. Reproduced with permission. ${ }^{139]}$

\section{PERSONALISINGTHE HEALTH EFFECTS OF RISKY DRINKING}

To design an optimally effective intervention to enhance motivation, the clinician should employ strategies that:

- Promote awareness of the risk associated with risky drinking.

- Offer the client acceptable and effective behaviour change options within an accepting and empathic atmosphere.

\section{Personalising the adverse health effects of risky drinking is viewed as integral in motivating drinkers to change their behaviour:}

Personalising health effects can be based on the following:

1. A discussion about the client's drinking-related symptoms and illnesses.

2. Feedback of medical information on the effects of alcohol consumption on the client's health, such as measures of liver function, for instance gamma-glutamyl transferase (GGT). [1]

Measures of liver function can indicate that alcohol is having a deleterious effect on the body. These tests are most appropriately conducted in medical settings because the necessary equipment is usually available. In non-medical treatment settings testing can be conducted elsewhere with the results forming the basis of a discussion between the clinician and the client. Results of liver tests should be explained to the client in a way that the client understands and can process. 
Drinkers who have developed symptomatic health problems are likely to react more strongly to such information than those who have not. However, this information can still have a motivating effect in "healthy" drinkers, because feedback of selected medical information at teachable moments can have a positive effect. ${ }^{[124]}$ The information gathered at the beginning of the treatment can also form a baseline against which later test results can be compared to demonstrate to the client the health improvements associated with a reduction in consumption of alcohol.

Although medical results within the normal range can have a motivating effect, they should be dealt with carefully. The client could, for example, take normal liver function to indicate that drinking is having no harmful effects. However, the clinician can explain that:

- Good health may not be maintained should the client continue to drink.

- Results in the normal range do not necessarily mean that drinking is not having a deleterious effect on health and functioning might have been higher if the person were not drinking excessively. ${ }^{[1]}$

\section{SUMMARY}

Motivational interviewing is advocated for use with problem drinkers who are expeniencing ambivalence or conflict about their drinking behaviour and modifying it. If the client is highly motivated to change, the clinician may find it useful to increase the client's level of self-efficacy and to reinforce their motivation by exploring what the client hopes to achieve by changing. Personalising the health effects of risky drinking is an integral part of the motivational interviewing approach. It is different from the simple provision of information on the negative effects of alcohol consumption which is thought to be much less likely to affect drinking behaviour.

\section{SUMIMARY OF EVIDENCE: MOTIVATIONAL INTERVIEWING}

Motivational interviewing is a moderately effective stand-alone treatment intervention which helps to reduce alcohol intake and to improve psychosocial outcomes.

Motivational interviewing can be delivered as a treatment prelude to increase motivation to change.

\section{LEVEL OF EVIDENCE}

I

II

STRENGTH OF RECOMMENDATION

\section{Strong}

treatment intervention.

Motivation is recommended particularly for clients

who are ambivalent about changing their behaviour.

Personalising information about the adverse health effects of risky drinking is recommended as a strategy to increase motivation to change. 


\section{Cognitive-behavioural interventions}

The term "cognitive behavioural" refers to an approach covering a range of strategies and techniques based on learning principles, based around the idea that modifying and re-learning behaviour is influenced by how people view themselves and others.

\section{- Cognitive behavioural interventions give the client a set of thinking and behaving strategies that can be used to assist in lifestyle change.}

Most treatment approaches that have been demonstrated to be effective in treating alcohol use disorders fall within the broad rubric of cognitive behavioural approaches. ${ }^{1140} \mathrm{In}$ addition to their effectiveness, advantages of cognitive behavioural interventions are that they:

- Are often detailed, making them amenable to training and dissemination.

- Can easily be developed into clear procedural guidelines which can be used to assess the quality of treatment delivery. ${ }^{[1]}$

In this way the use of these techniques makes it easier to monitor the activities that occur in practice and thereby assure the integrity of treatment implementation.

On the basis of evidence supporting the effectiveness of cognitive behavioural interventions, well articulated procedures and protocols for implementation, and acceptance and popularity within the Australian treatment community, cognitive behavioural interventions are likely to provide an effective, practical basis for the treatment of risky drinkers and alcohol dependence. They are delivered in a form that allows for a rapport and trust to be developed between clinician and client in a non-confronting and empathic atmosphere. These approaches are compatible with other interventions such as pharmacological interventions and motivational interviewing techniques.

\section{SUMMARY}

It is recommended that a range of cognitive behavioural interventions (described below) be adopted in the management of alcohol dependence and associated problems.

\section{KEY POINTS}

- Cognitive behavioural interventions encompass a range of strategies and techniques including skills training, behavioural self-management, cognitive restructuring, cue exposure and behavioural couples therapy.

- Cognitive behavioural interventions aim to give the client a set of thinking and behaving strategies that can be used change problematic behaviours.

SUMMMARY OF EVIDENCE: COGNITIVE BEHAVIOURAL INTERVENTIONS

Cognitive behavioural interventions are effective treatments that reduce alcohol consumption and improve psychosocial outcomes.

\section{LEVEL OF EVIDENCE}

Various interventions that fall under the umbrella of cognitive Strong

behavioural interventions should be implemented in treatment settings. 


\section{Skills training}

Skills training is a form of cognitive behavioural treatment intervention. It involves teaching people social skills that might help them function without the use of alcohol. There is consistent evidence that skills training helps to reduce alcohol consumption in both the short term and the long term among risky drinkers and alcohol dependent persons.

Skills training can be used to compensate for skills deficits that have led to the use of drinking as a coping strategy. It has also been linked to Marlatt and Gordon's model of relapse prevention and may help clients to deal with high risk situations. ${ }^{[141]}$ Skills training is recommended for use with clients who have a high risk of relapse (see Chapter 9: Relapse prevention). There are various skills training approaches briefly described below, and although there is some overlap in the techniques in each of these procedures, there is sufficient difference in procedure and purpose to divide them into separate sections. These procedures are of most benefit to those clients who lack the relevant skills, and should be offered to those clients whom clinicians perceive as lacking relevant skills. Although it is often difficult to ascertain which skills clients possess, particularly without appropriate assessment, asking clients what problems they experience and how they cope with them can expose the areas in which clients are lacking social skills.

Skills training can be used with clients aiming at moderation or abstinence. Several approaches may be appropriate for the one person and skills training approaches are often offered in combination. The intervention chosen should be relevant to the client's needs. Training in social skills and other coping skills will require some commitment on the part of the client as these methods are enhanced when the client actively participates in therapy and continues to practise outside therapy. It is inappropriate to train a client in skills in which they are already adept.

In skills training an emphasis is placed on ensuring that clients:

- Learn to listen and to communicate effectively with others

- Give and receive compliments and criticism

- Learn to refuse unwanted requests

- Learn to communicate non-verbally and verbally

- Begin to build a social support network ${ }^{[142]}$

A key element of skills training is breaking down behaviour into component steps. This is essential if learning new ways of behaving is to occur, because most behaviours are engrained and occur without the person's knowledge of what he or she is doing.

\section{PROBLEM-SOLVING SKILLSTRAINING}

Problem solving skills training provides clients with a set of general skills that may assist in resolving life problems which may threaten their commitment to change their drinking behaviour. ${ }^{[1]}$ The basics of problem-solving skills training do not take long to teach, but they require practice to be effectively learnt. Therefore, problem-solving skills should be practised, refined and reinforced in treatment both after they have been taught and by the client at home.

- Problem solving skills training is appropriate for all clients, regardlless of their drinking goal, and is suitable for implementing in either an individual or group session, although leaming through practice is probably facilitated by working in groups of clients rather than on an individual basis. 
The goals of problem solving skills training are to teach clients to:

- Recognise when a problem exists

- Generate a variety of possible solutions

- Select the most appropriate option

- Generate a plan for action

- Evaluate the effectiveness of the selected approach ${ }^{[142]}$

\section{ASSERTIVENESS SKILLSTRAINING}

An inability to express feelings to others in an appropriately assertive and clear way can create anxiety, frustration and anger, which can contribute to a return to risky drinking. Assertiveness training is likely to have an important role in the management of interpersonal situations that have the potential to produce negative mood states and thereby predispose the drinker to relapse to drinking. The procedure has evidence of efficacy, especially if "booster" sessions are used to ensure that the learnt skills are maintained, see Connors $2001{ }^{[143]}$.

\section{Assertiveness skills are recommended particularly for clients who have difficulty in expressing their emotions.}

In drink refusal skills training the client learns that he or she has the right to express personal opinions and feelings, to request that other people change behaviour that affects the client, and to accept or reject other people's requests. Underlying this approach is the development of selfrespect and respect for the rights of others. Assertive skills training typically focuses on developing overt behavioural skills through repeated role playing and practice of "difficult" situations, and the concurrent change in the person's thinking as he or she develops the confidence and ability to be assertive.

\section{- Assertiveness skills are equally effective for goals of moderation or albstaining. \\ - Group settings are ideal for teaching this technique as role-play is an essential part of leaming assertiveness skills.}

\section{COMMUNICATION SKILLSTRAINING}

Communication skills training shares substantial overlap with assertiveness skills training and the two can and often are taught together. Communication skills can help to reduce feelings of social embarrassment and tension. Communication skills training aims to teach the client how to:

- Start and continue conversations in a comfortable manner.

- Cope with silences and pauses in conversations.

- Interpret social cues.

- Actively listen to others.

- Comfortably communicate personal feelings and opinions.

C Communication skills training can be used regardlless of clients' drinking goals. Communication skills training is best implemented in group settings, where clients can practice communication skills through role-play and modelling. 


\section{DRINK REFUSAL SKILLSTRAINING}

Drink refusal skills training teaches the drinker to confidently and assertively say no to offers of alcohol or to social pressure to drink. Social pressure to have a drink can be substantial, and the client needs to develop and practise strategies that allow him or her to respond effectively.

\section{Drink refusal skills training should be offered to all clients who indicate a lack of confidence in dealing with social pressure to drink.}

In the training an emphasis is placed on speaking directly to the person making the offer or placing pressure on the drinker. Drinkers are given instructions about how to suggest alternatives to alcohol, dealing with pressure in an appropriately assertive way, and not feeling guilty about refusing. Drink refusal skills training can be used for clients with a goal of either abstinence or moderation. For clients with a goal of abstinence, drink refusal skills are a very important aspect of relapse prevention. They can also be helpful for clients with a goal of moderation to keep to predetermined limits in the face of temptation or pressure to over drink. Drink refusal skills can be taught on an individual basis, although group settings are more appropriate as they give clients the opportunity to practise the skills taught and to learn from one another in role-play activities.

\section{Drink refusal skillss training can be used for clients with a goal of either albstinence or moderation.}

Drink refusal skills training can also be used with behavioural self-management (discussed below), to assist the client to deal with situations in which alcohol is present. This procedure has been incorporated into many interventions which have shown sustained effects in reducing drinking.

\section{RELAXATIONTRAINING AND STRESS MANAGEMENT SKILLSTRAINING}

Relaxation and stress management procedures represent a wide range of interventions that attempt to train the client in methods to deal with daily tension that may be associated with risky drinking. The tension is often physical tension, but it can also be psychosocial or mental tension. There are a number of relaxation procedures available for this.

In this set of procedures the client learns techniques for inducing relaxation that might include deep-muscle relaxation, relaxing imagery, and letting go of physical stress. There is evidence of the efficacy of these procedures, both generally for reducing anxiety and tension, and more specifically in the context of programs aimed at reducing drinking.

\section{- Relaxation training is appropriate when clients have mentionedl or displayed signs of tension. \\ - Relaxation training is suitable for clients with abstinence or moderation goals, and it can be implemented in individual or group settings.}

The goals of relaxation and stress management training are to teach clients to:

- Recognise when tension is present.

- Relax his/her body when tension is detected to reduce tension.

- Use mental imagery or meditative methods to reduce psychosocial tension. 
Skills training approaches are advocated as appropriate interventions for clients with varying severity of alcohol problems where assessment shows the need for such intervention. Included are:

- problem solving skills training

- drink refusal skills training

- assertive skills training

- communication skills training

- relaxation training and stress management skills training.

\title{
SUMMMARY OF EVIDENCE: SKILISTTRAINING
}

LEVEL OF EVIDENCE

Skills training can be used to compensate for skills deficits and help to reduce alcohol consumption and increase psychosocial outcomes for those who have an alcohol use disorder.

Skills training is effective in both the long and short term among risky and dependent drinkers.

Skills training is appropriate for goals of moderation and abstinence.

Different types of skills training are more effective in combination than in isolation.

II

II

II

\section{RECOMMENDATIONS: SKILLSTRAINING}

STRENGTH OF RECOMMIENDATION

Skills training should be offered to clients who lack Strong the relevant skills.

Problem solving skills training, assertiveness skills training, communication skills training, drink refusal skills training, and relaxation and stress management skills trainings are recommended to reduce or eliminate alcohol consumption among risky and dependent drinkers.

Although appropriate for individual settings, skills Moderate should be implemented in group settings to allow for role-playing and modelling.

Skills training is recommended for clients who have a high risk of relapse.

\author{
Strong
}




\section{Behavioural self-management}

Behavioural self-management is a cognitive behavioural treatment intervention. Behavioural selfmanagement training involves a series of strategies such as:

- self-monitoring

- setting drinking limits

- controlling rates of drinking

- identifying problem drinking situations

- self-reward for limited drinking

\section{The goal of behavioural self-management is to teach clients specific skills so that they can reduce drinking to stable, low-risk levels.}

Behavioural self-management is intended for those clients who wish to cut down rather than abstain from drinking. However, these procedures could be usefully taught as relapse prevention strategies to drinkers who have a goal of abstinence. This procedure might be especially useful for those drinkers whose lives are enmeshed in a drinking culture where non-drinking is extremely unlikely.

In a typical behavioural self-management intervention, the client learns about the concept of blood

alcohol concentration and the recommended levels of alcohol consumption, which have been defined for Australia by the National Health and Medical Research Council as: ${ }^{[3]}$

for men,

- No more than six drinks on any one day $(60 \mathrm{~g})$, for risk of harm in the short term, and

- No more than four standard drinks of alcohol per day (40g), or 28 standard drinks (280g) per week for risk of harm in the long term.

for women,

- No more than four standard drinks on any one day, for risk of harm in the short term, and

- No more than two standard drinks of alcohol per day (20g) or 14 standard drinks (140g) per week for risk of long-term harm.

A plan for reducing the client's drinking should be formulated, based on drinking patterns as identified through self-monitoring.

Specific strategies for reducing consumption through behavioural self-management include:

- Keeping a diary to monitor how much alcohol they drank, where they drank, what time of the day they drank, who they drank with, and how they were feeling at the time.

- Setting a limit on the number of standard drinks to be consumed on a given occasion.

- Timing how long it takes to consume a drink and placing a minimum time on each drink.

- Taking small sips to reduce the amount of alcohol consumed and to allow each drink to last longer.

- Resting in between sips, by putting the glass down on the table, rather than holding it continuously. 
- Diluting drinks with non-alcoholic mixers, or drinking low alcohol beer

- Alternating alcoholic drinks with non-alcoholic drinks such as soft drinks, mineral or soda water.

- Avoiding participation rounds, or sitting out one or more rounds as necessary.

This procedure requires that the client is familiar with the definition of a standard drink as it applies to different beverages (see Table 8.4).

One potential problem arising from this procedure is that drinkers for whom abstinence is advisable may see this strategy as a means of "safe" drinking. If drinkers with a goal of abstinence are warned that these procedures should only be used if there is a lapse to drinking, then there may be a role for behavioural self-management in interventions for those with a goal of abstinence. If there is doubt about a client's ability to deal with this information appropriately, it is recommended that the technique not be used.

TABLE 8.4: STANDARD UNITS OF ALCOHOL

\begin{tabular}{lccc}
\hline Type of Alcohol & Volume & $\begin{array}{c}\text { Percentage of Alcohol } \\
\text { by Volume } \%\end{array}$ & $\begin{array}{c}\text { Number of } \\
\text { Standard Drinks }\end{array}$ \\
Full Strength Beer & $425 \mathrm{ml}$ & 4.5 & 1.5 \\
Full Strength Beer & $375 \mathrm{ml}$ & 4.5 & 1.3 \\
Full Strength Beer & $285 \mathrm{ml}^{*}$ & 4.5 & 1.0 \\
Mid Strength Beer & $425 \mathrm{ml}$ & 3.4 & 1.13 \\
Mid Strength Beer & $375 \mathrm{ml}$ & 3.4 & 1.0 \\
Mid Strength Beer & $285 \mathrm{ml}$ & 3.4 & 0.8 \\
Light beer & $425 \mathrm{ml}$ & 2.8 & 1.0 \\
Light beer & $375 \mathrm{ml}$ & 2.8 & 0.8 \\
Light beer & $285 \mathrm{ml}$ & 2.8 & 0.5 \\
Wine & $100 \mathrm{ml}$ & 12.5 & 1.0 \\
Fortified Wine & $60 \mathrm{ml}$ & 21.0 & 1.0 \\
Spirits & $30 \mathrm{ml}$ & 42.0 & 1.0 \\
Premixed Alcoholic Soda & $300 \mathrm{ml}$ & 5.0 & 1.2 \\
\hline
\end{tabular}

*NSW, WA, ACT = Middy; QLD, VIC, TAS = Pot; NT = Handle; SA = Schooner

Note: Alcohol content varies according to brand. 


\section{SUMMARY}

Behavioural self-management is recommended as a treatment strategy when both client and clinician agree that moderation is the appropriate treatment goal. If used in the context of a goal of abstinence, it should be presented as a strategy to cope with a temporary lapse to drinking.

\begin{tabular}{l|c}
\hline SUMMARY OF EVIDENCE: BEHAVIOURAL SELF-MANAGEMENT & LEVEL OF EVIDENCE \\
\hline $\begin{array}{l}\text { Behavioural self-management is a moderately effective } \\
\text { strategy to reduce alcohol consumption among risky and } \\
\text { dependent drinkers with a goal of moderation. }\end{array}$ & II \\
\hline RECOMMENDATIONS: BEHAVIOURAL SELF-MANAGEMENT & $\begin{array}{l}\text { STRENGTH OF } \\
\text { RECOMMENDATION }\end{array}$ \\
\hline $\begin{array}{l}\text { Behavioural self-management should be offered to clients } \\
\text { with a goal of moderation. }\end{array}$ & Strong \\
\hline $\begin{array}{l}\text { Behavioural self-management should be taught to clients } \\
\text { who are extremely unlikely to stop drinking. }\end{array}$ & Strong \\
\hline $\begin{array}{l}\text { The client should be introduced to the National Health and } \\
\text { Medical Research Council's Alcohol Guidelines. }\end{array}$ & Strong \\
\hline $\begin{array}{l}\text { Clients should be taught about standard drink sizes to assist } \\
\text { in monitoring levels of consumption. }\end{array}$ & Strong \\
\hline
\end{tabular}

\section{Cognitive restructuring}

Cognitive restructuring is a cognitive behavioural intervention. Cognitive restructuring works with current beliefs and attitudes of the client and is designed to help the client identify and change irrational or counterproductive beliefs, especially where these contribute to continued drinking. Cognitive restructuring should be viewed as a collaborative process between the clinician and client. It can be offered in a group or individual setting. Cognitive restructuring is not recommended for clients with cognitive deficits because of its abstract nature.

Cognitive restructuring has been shown to be effective when combined with other strategies, particularly skills training. In addition to its effectiveness in treating alcohol problems, cognitive restructuring techniques have been shown to be effective in the treatment of other disorders, particularly anxiety and depression ${ }^{[144]}$, which occur at significant rates in problem drinkers.

\section{The goals of cognitive restructuring are for clients to recognise when they are thinking in a way that is likely to lead to drinking and to intemupt and challenge these thoughts. Cognitive restructuring is appropriate for clients with a goal of either moderation or albstinence.}

Cognitive restructuring needs to be practised during treatment sessions so that the client can use 
the skills effectively after treatment ends.

There are several areas in which the clinician can help the client identify and change irrational beliefs, attributions and self-statements. These include beliefs relating to drinking behaviour, relapse, emotional discomfort, and oneself.

Cognitive restructuring aims to encourage clients to base their beliefs about drinking and about their self-worth in the experiences of reality. The restructuring involves active participation by the client, and the development of new perspectives should reflect the client's own values, not those of the clinician.

\section{SUMMARY}

Cognitive restructuring is designed to help the client identify and change irrational or counterproductive beliefs, attributions and self-statements, especially where these contribute to continued drinking. Cognitive restructuring procedures are recommended as part of more comprehensive interventions that rely upon providing the client with specific skills. The areas where cognitive restructuring can prove useful include erroneous views about drinking behaviours, about oneself, and about relapse.

SUMMARY OF EVIDENCE: COGNITIVE RESTRUCTURING

Cognitive restructuring is a moderately effective technique to assist in reducing alcohol consumption among risky drinkers and alcohol dependent people.

Cognitive restructuring works equally well for goals of moderation and dependence.

\section{LEVEL OF EVIDENCE}

II

II

STRENGTH OF RECOMMENDATION

RECOMIMENDATIONS: COGNITIVE RESTRUCTURING

Strong

recommended as part of more comprehensive

interventions that rely upon providing the client

with specific skills.

Cognitive restructuring is not recommended for clients with cognitive deficits.

Cognitive restructuring should be practised throughout the treatment process.

\section{Strong}

Strong 


\section{Cue exposure}

Cue exposure is a cognitive behavioural intervention. Cue exposure is based on the associative learning principle ${ }^{[145]}$, which assumes that people, places and events that regularly precede drinking become associated with the pleasant effects of alcohol, and alcohol consumption becomes a conditioned response to these cues. Alcohol-related cues include the sight and smell of an alcoholic drink, mood states or situations in which drinking has previously occurred, and people, places and times that have previously been associated with the pleasant effects of alcohol. Two models classical learning theory, and social learning theory - have been used to explain the relationship between alcohol-related cues and relapse to drinking. Treatment can therefore vary according to the theory on which the treatment is based.

According to classical learning models, the client only needs to be exposed to alcohol repeatedly without being allowed to drink in order to prevent the usual drinking response to those cues. Social learning theory models assume that the chance to practise coping skills in the presence of alcohol cues is important in reducing relapse. Consequently, some cue exposure approaches focus on pure exposure to alcohol cues, whereas other approaches include coping skills training in the presence of alcohol cues. ${ }^{[16]]}$ Most studies of alcohol use have offered adjunct treatment in addition to cue exposure.

Another variation among different approaches is the nature of the cues that are used during the exposure trials. Because of the diversity of the settings associated with drinking, it is very difficult to expose drinkers to all possible real-life settings associated with drinking. Thus, some treatments use imaginary exposure, and ask clients to imagine a situation in which drinking has occurred previously. Many cue exposure treatments use an alcoholic beverage as a cue, since the sight and smell of alcohol is a cue that every drinker is exposed to before drinking. Some approaches, particularly those with a goal of moderation rather than abstinence, use the consumption of a small amount of alcohol as the cue. Thus, the choice of cues is based on treatment goal - either moderation or abstinence - and the constraints of the setting in which treatment is being conducted, that is, the extent to which reallife settings can be replicated. ${ }^{[146]}$

The goal of cue exposure is to decrease the likelihood of a relapse to drinking by either:

- Decreasing the strength of association between alcohol-related cues and the urge to drink, or

- By increasing the use and effectiveness of coping skills by drinkers, when confronted with alcohol-related cues in real-life situations.

Cue exposure therapy is appropriate where the goal of treatment is either moderation or abstinence. Cue exposure therapy usually consists of between six and twelve sessions, each session lasting between 50 and 90 minutes. Treatment sessions can be run daily or can be spaced across a number of days. ${ }^{[147]}$

The length of time to which a client is exposed to a cue is determined either by:

- A set amount of time which is pre-determined before the start of therapy (e.g. smelling a glass of alcohol for three minutes).

- When the participant's self-reported craving or urge level drops to half the peak intensity experienced during exposure to the cue.

Cue exposure is a specialist treatment intervention and should only be offered by suitably qualified professionals. 


\section{KEY POINTS}

- Two models - classical learning theory models, and social learning theory - have been used to explain the relationship between alcohol-related cues and relapse to drinking.

- Some cue exposure approaches focus on pure exposure to alcohol cues, whereas other approaches include coping skills training in the presence of alcohol cues.

\begin{tabular}{|c|c|}
\hline SUMMARY OF EVIDENCE: CUE EXPOSURE & LEVEL OF EVIDENCE \\
\hline $\begin{array}{l}\text { Cue exposure is a moderately effective } \\
\text { intervention for the treatment of alcohol } \\
\text { dependence and risky drinking. }\end{array}$ & II \\
\hline RECOMMENDATIONS: CUE EXPOSURE & STRENGTH OF RECOMMENDATION \\
\hline $\begin{array}{l}\text { Cue exposure should consist of six to twelve } \\
\text { sessions of } 50 \text { to } 90 \text { minutes. }\end{array}$ & Moderate \\
\hline $\begin{array}{l}\text { Cue exposure should only be offered by } \\
\text { treatment specialists who have appropriate } \\
\text { training in the strategy. }\end{array}$ & Strong \\
\hline
\end{tabular}

\section{Couples and family therapy}

Although couples and family therapy are often grouped together as a single intervention, there are a number of reasons why they should be viewed as distinct interventions.

Couples therapy involves the partner of the problem drinker, while family therapy involves the partner in addition to other family members.

Family therapy requires a clinician with specialist skills and training in the provision of family therapy. It is unlikely that suitably skilled clinicians will be available in most drug and alcohol agencies.

There is scant research on the effectiveness of family therapy in the area of alcohol and substance abuse. Thus, this section focuses on couples therapy, in particular, behaviourally-oriented couples therapy.

Data suggests that problem drinking is associated with negative functioning, not only for the person with the alcohol problem, but also for the non-alcoholic partner and for the relationship. Evidence that marital/relationship problems precede heavy alcohol use is limited, but relationship problems have been associated with relapse after treatment.

Research on behaviourally-oriented couples therapy indicates that:

- Intervening at the couples level can motivate an initial commitment to change in the alcohol dependent person who is unwilling to seek help.

- Behavioural couples therapy alone or in addition to individual treatment can produce better relationship and/or drinking outcomes during the six months following treatment than approaches which do not include the partner. ${ }^{[148]}$ 
Behavioural couples therapy is a cognitive behavioural treatment intervention. It is only appropriate when there is agreement between the client, the client's partner and the clinician that the partner's involvement is likely to be beneficial. It is very important to avoid blame in couples therapy. The therapy should emphasise that drinking is the problem, and focus on drinking behaviour as the problem, which needs to be fixed. It is not designed to address entrenched relationship problems or to counteract violence.

Couples therapy includes several treatment elements, which are discussed elsewhere in this section. These include skills training for partners, cognitive restructuring and behavioural self-management. Different models place emphasis on different elements of the treatment. Implicit in the model is the need for detailed assessment to determine the primary factors contributing to the problem, the skills and deficits of the individual and the couple, and sources of motivation to change behavioural patterns.

The overall goal of couples therapy is to improve the couple's relationship and communication in a way that will strengthen the capacity and commitment to achieve and sustain a change in drinking. In particular, couples therapy aims to:

- Change alcohol-related interactional patterns and develop interactions that support a change in drinking behaviour.

- Help the couple confront and resolve relationship conflicts without the alcohol dependent person resorting to the use of alcohol.

- Mend relationship problems that have been aggravated as a result of the alcohol problem.

- Help the couple develop shared activities that are rewarding and do not involve alcohol. ${ }^{[142]}$

As this therapy requires partners to work together, it is most appropriate for couples with moderate to low problems in their relationship, couples who are living together, are at least high school educated and are employed. ${ }^{[149]}$

\section{BEHAVIOURAL COUPLESTHERAPY}

There are four guiding components of couples therapy. ${ }^{1500}$ The first is aimed at the individual rather than the couple. This component helps the client assess potential and actual reinforcers for continued drinking, and in assessing the negative consequences of continued use. At this level, skills training, behavioural self-management and cognitive restructuring are important elements of the intervention. These interventions are explained in more detail above.

The second component of couples therapy revolves around the coping skills of the partner. This component involves teaching the partner ways to deal with risky drinking. These skills may include learning new ways to discuss drinking, learning new responses to the partner's drinking and behaviour when drinking, or individual skills to enhance his or her own individual functioning.

The third component of couples intervention focuses on interactions between the two partners, around both drinking and other issues. In this component, basic communication and problem-solving skills are taught to the couple. Topics relating to the drinking behaviour of the client are discussed with the clinician present as a vehicle for teaching communication skills.

The fourth component focuses on other social systems in which the drinker and partner are currently involved. Clients and partners are helped to identify situations and persons who appear to be associated with heavy drinking, and also to identify persons and situations that would be supportive of abstinence or moderate drinking, depending upon the goal of treatment. 


\section{- Clinicians who deliver behavioural couples therapy should be suiltably trained in the delivery of this intervention. \\ - The interventions they deliver should be supported by research.}

\section{BEHAVIOURAL MARITALTHERAPY}

Behavioural marital therapy (BMT) is a particular type of behavioural couples therapy on which much of the research examining behavioural couples therapy has been based. This is the approach developed by O'Farrell and his colleagues. ${ }^{[149]}$ Two alcohol-focused methods have been used to reduce alcohol consumption in this approach:

- A behavioural contract between the alcohol dependent person and the partner to maintain disulfiram ingestion.

- "Alcohol-Focused Spouse Involvement" which rearranges reinforcement contingencies in the family to decrease family member behaviours that trigger or enable drinking and to increase positive reinforcement for sobriety. ${ }^{[199]}$

BMT also works on general marital issues including:

- Direct instigation of positive couples and family activities.

- Teaching of communication and conflict resolution skills.

SUMMARY OF EVIDENCE: COUPLES AND FAMILYTHERAPY

LEVEL OF EVIDENCE

Couples therapy is moderately effective in reducing

alcohol consumption and improving psychosocial outcomes.

Intervening at the couples level with a non-alcohol dependent partner can motivate an initial commitment to change in the alcohol dependent person who is unwilling to seek help.

Behavioural couples therapy alone or in addition to individual treatment can produce better relationship and/or drinking outcomes during the six months following treatment than approaches which don't include the partner.

Behavioural couples therapy should emphasise that drinking

Strong is the problem, and focus on drinking behaviour as the problem which needs to be fixed.

Behavioural couples therapy should not address entrenched relationship problems or be used to counteract violence.

Strong

Behavioural couples therapy is recommended for couples with Fair moderate to low problems in their relationship, couples who are living together, are at least high school educated and are employed.

Clinicians who deliver behavioural couples therapy should be Strong suitably trained in the delivery of this intervention. 


\section{Self-guided materials}

Self-guided materials have been available to help individuals to change problematic drinking behaviours since the 1970s. ${ }^{[151]}$ Self-guided materials can be used either with other treatment interventions or as a stand-alone intervention. There are now several self-help manuals available for use by drinkers who wish to cease or cut down drinking without the aid of professionals ${ }^{1522}$ ${ }^{153}$ and there is evidence that the use of these manuals is associated with a marked diminution of drinking. ${ }^{[154,155]}$

Self-help manuals have been recognised as an important addition to treatment interventions as they may target a portion of the population that does not usually receive treatment. These drinkers are likely to include women and those who live in remote or isolated areas. Other attractive components of self-help manuals include: anonymity, low time commitment, the absence of travel and specific appointments, and the absence of costs. ${ }^{[156]}$

Brief guidelines provided by the West Australian Alcohol and Drug Authority are thought to be useful for drinkers who require basic information or hints on reducing consumption of alcohol to moderate levels. ${ }^{[152]}$ More detailed books and booklets have been produced and are recommended for general distribution. The Guide to healthier drinking: A self-help manual prepared for the Alcoholscreen Team ${ }^{[157]}$ has straightforward coverage of practical and understandable approaches to moderated drinking. For those drinkers who are pursuing the goal of abstinence, appropriate self-help materials are less apparent, although many of the strategies covered within the texts mentioned above are likely to be of value.

Although self help materials have some support, and have an important place in the treatment of alcohol problems, applying self-help approaches is not always sufficient to change behaviour. The use of self-help materials is most appropriate for risky drinkers rather than those suffering from alcohol abuse or dependence. Self-help books can be used when there is professional intervention as they enhance the messages that are provided through more formal face-to-face intervention.

Some self-help books can be written at too high a reading age for some drinkers, and they need to be altered for presentation to non-English speaking people. More attention is needed on these aspects of presentation of self-help materials to ensure that the messages about altering alcohol consumption reach all parts of the Australian community.

\section{SUMMARY}

Self-help materials should be well advertised and distributed in the community for drinkers who do not wish to be involved in professional interventions or in self-help groups. The materials are also important resources for clinicians and professionals to use in formal intervention. Further materials of this type, suitable for people from non-English speaking backgrounds, and for people with a low reading age, need to be developed. Computer and internet-based self-help materials may provide helpful materials to a population of problem drinkers who may not ordinarily seek treatment. 
There is evidence that the use of self-help manuals is

II associated with a marked diminution of drinking.

Self-help manuals are an important addition to treatment interventions as they may target a portion of the population who do not usually receive treatment.

\title{
RECOMMENDATIONS: SELF-HELP MANUALS
}

II

\begin{abstract}
Self-help manuals should be made readily available to the general public to assist drinkers who wish to cease or cut down drinking without the aid of face-to-face help.

Self-help materials are most appropriate for risky drinkers rather than those suffering from alcohol abuse or dependence.
\end{abstract}

Self-help manuals should be modified to suit people Strong from non-English speaking backgrounds and those with a low reading age.

Computer and internet-based self-help materials should continue to be developed as they may provide helpful materials to a population of problem drinkers who may not ordinarily seek treatment.

\section{Standardised versus tailored treatment}

The goal of standardised treatment, through the provision and use of treatment manuals, is to improve the quality of treatment by standardising it in written form.

Advantages of standardised treatments are that:

- They are often empirically validated.

- Because they are structured and time limited, they are often more focused than individualised therapy.

- They are easier to disseminate than other clinical methods, resulting in greater ease in learning specific treatment strategies and acquiring skill in using them.

- They are useful in training and supervision. ${ }^{158]}$

In addition, according to a meta-analysis, clinician effects on treatment outcome decrease when adherence to treatment increases. ${ }^{[136]}$ This suggests that implementing treatment manuals into clinical practice results in standard practice being conducted by clinicians, decreasing variability in outcome that is not attributable to treatment. This should result in increased effectiveness as treatments are being delivered as intended. 
Treatment manuals are often criticised by clinicians, and there has been some resistance to implementing them in clinical settings. Perceived disadvantages of treatment manuals are that:

- They are conceptually at odds with fundamental principles of cognitive behavioural therapy, which emphasises the importance of tailoring interventions to each client's needs.

- They preclude individual case formulation and undervalue the importance of assessment and case formulation.

- They undermine clinicians' clinical artistry.

- They apply primarily to research samples which differ from the clinical samples.

- They promote particular schools of psychosocial therapy. ${ }^{[158]}$

Although these objections are not unfounded, they do not necessarily impede clinical judgement. [159]

Manual-based treatment does not mean that therapy is not individualised. Although strict adherence to treatment manuals is often required in research studies, in clinical practice clinicians can be more flexible. Manuals can and should:

- Allow for tailoring the timing of the intervention to the needs of each client.

- Offer multiple techniques, all of which need not be included in treatment for every client.

- Allow for interrupting the manual if a more salient problem arises which is not addressed by the techniques in the manual. ${ }^{[159]}$

For individual case formulation, numerous studies have shown that clinicians make cognitive biases in drawing inferences and judgements about clients. Clinicians' confidence in their judgements is unrelated to their accuracy, and they tend to overestimate their ability to accurately assess clients and to evaluate the success of treatment. [159] A greater reliance on standardised treatments is a means of minimising errors to which clinical judgement is vulnerable.

The importance of developing rapport and building a positive therapeutic alliance is no less important in manual-based therapy than in conventional therapy. Rather than undermining clinician's clinical artistry, Wilson [159] argues that manual-based therapies require specific clinical skills in developing effective therapeutic alliances with clients.

Despite disagreement about the use of treatment manuals, and possible limitations resulting from implementing them in practice, the use of manuals is advocated as they are thought to promote the delivery of effective treatment. Furthermore, many of the criticisms of treatment manuals do not necessarily apply in clinical practice.

If clinicians are to be maximally effective, there must be detailed knowledge of the treatments to be provided, and there should be a protocol to follow. This approach also assists greatly in the performance and evaluation of new staff members, who may otherwise be unsure and confused about what is required in treatment and who may bring inappropriate components of their background into the clinical setting. Where clinicians are not clear about the content or aims of treatment, clients will also be uncertain and the overall effort is likely to become confused and less effective. Clinicians should be adequately trained in the procedures involved. 


\begin{tabular}{l|c}
\hline SUMMARY OF EVIDENCE: STANDARDISED VERUS TAILOREDTREATMENT & LEVEL OF EVIDENCE \\
\hline $\begin{array}{l}\text { The use of treatment manuals decreases clinician effects on } \\
\text { treatment outcome. }\end{array}$ & I \\
\hline RECOMMENDATIONS: STANDARDISED VERSUS TAILORED TREATMENT & $\begin{array}{c}\text { STRENGTH OF } \\
\text { RECOMMENDATION }\end{array}$ \\
\hline $\begin{array}{l}\text { Treatment procedures should be clearly specified in a } \\
\text { written form. }\end{array}$ & Strong \\
\hline $\begin{array}{l}\text { Clinicians should be adequately trained in the } \\
\text { procedures involved. }\end{array}$
\end{tabular}


Chapter 9: Relapse prevention 


\section{Chapter 9: Relapse prevention}

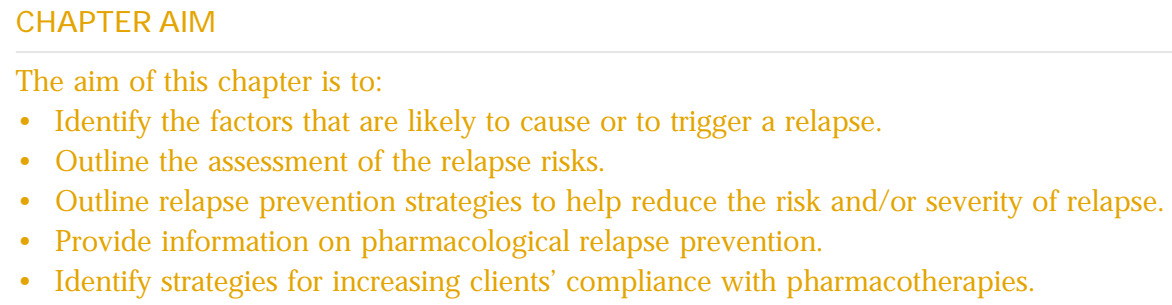

The evidence for using relapse prevention strategies with alcohol dependent clients is strong. Because relapse is one of the most important problems for those attempting to overcome addictive behaviours, addressing the problem is an essential aspect of treatment and one that poses major difficulties in the area of drug and alcohol dependence. There is a substantial relapse rate within the first year after treatment of about 60 percent when relapse is defined as a return to problem drinking. ${ }^{[160]}$

Relapse prevention aims to maintain long term abstinence or moderate drinking behaviours, and to decrease the severity of relapse if it does occur. The conceptual model of relapse prevention views relapse as a natural part of the process of change: lapses and relapses are viewed as opportunities for clients to understand their behaviour and develop new skills to deal with high-risk situations. [161] Traditionally, relapse prevention refers to the model of relapse prevention developed by Marlatt and colleagues. ${ }^{[141]}$ This model includes a variety of cognitive and behavioural approaches designed to target each step of the relapse process. These approaches include specific intervention strategies that focus on the immediate determinants of relapse as well as global self management strategies that focus on the covert antecedents of relapse. ${ }^{1623}$ Both the specific and global strategies fall into three main categories: skills training, cognitive restructuring and lifestyle balancing. Such strategies are designed to address the immediate precursors of relapse and involve the training of coping skills, challenging positive outcome expectancies associated with alcohol use and how to cope with lapses. Effective relapse prevention also requires that longer term factors in relapse are addressed.

\section{Identifying factors associated with relapse}

The factors most frequently, but not exclusively, ${ }^{4}$ associated with relapse to alcohol and drug use are thought to include: ${ }^{[163,160]}$

1. Negative emotional states such as frustration, anger, anxiety, depression, boredom (this association may be stronger for women than men). Negative emotions might also be the result of other factors such as social isolation, lack of coping skills, and the negative effect of alcohol on interpersonal, social and occupational functioning.

2. Inadequate coping skills: when faced with high risk situations, the clients coping skills determine whether relapse occurs or not.

3. Social isolation and family factors: interpersonal conflicts within marital, social, family or work relations, being unemployed.

4. Alcohol involvement before treatment.

5. Craving post-treatment.

6. Beliefs: self-efficacy ratings and disease model beliefs, that is, the view of alcohol dependence as a loss-of-control disease. 


\section{Strategies for assessing relapse risk}

The overall goal of the specific intervention strategies is to teach the drinker to recognise and cope with the high-risk situations that might precipitate a lapse, and to modify the drinker's reaction to a lapse so that it does not develop into a full-blown relapse. The first step taken is to assist the client to recognise those particular high-risk situations that might precipitate a relapse. Typically this involves reviewing a list of common relapse situations, identifying those that are likely to cause difficulty for the drinker, and devising methods to either avoid these situations or cope with them without drinking. Self-monitoring of drinking-related behaviours before cessation can provide information about specific risk situations, and can highlight the situations and skills deficits associated with risky consumption. It is also important to recognise that drinkers can relapse in unexpected situations. This possibility should be discussed with the drinker, and strategies developed that will allow the drinker to manage these situations if they arise. However, the drinker should be encouraged initially to avoid high-risk situations.

Qualitative information can be obtained by clinical interview by asking Marlatt's original questions:

1. In your own words, what is the main reason why you drink?

2. Are there any other reasons why you drink, which you consider important? If Yes, what are they?

3. Do you have any inner thoughts or emotional feelings, or things within you as a person which "trigger off" your need or desire to take a drink at a particular moment in time?

4. Are there any particular situations or set of events, things which happen to you in the outside world which would be most likely to make you feel like having one or more drinks? [164]

Clients can be asked about the circumstances under which they have relapsed or drank heavily in the past. Other questions should explore the relapse factors identified above, for example exploring the client's beliefs about alcohol dependence as a disease, beliefs about their capacity to avoid relapse and cope with lapses, their strategies for coping with high-risk situations, mood, and social/family support.

Psychometric instruments include:

- The Reasons for Drinking Questionnaire (RFDQ) can also be used to identify antecedents of relapse for individual clients. ${ }^{165]}$

- The Inventory of Drug Taking Situations (IDTS) assesses situations in which the client has consumed heavily or relapsed in the past. Its companion is the Drug Taking Confidence Questionnaire (DTCQ) which assesses the client's self-efficacy about high-risk situations, one of the factors associated with relapse. ${ }^{[166]}$

- The Situational Confidence Questionnaire ${ }^{[166]}$ provides a basis for the identification of high-risk situations.

- The Coping Behaviours Inventory ${ }^{[167]}$ to help identify the client's coping skills. 


\section{Reducing the risk of relapse}

Relapse prevention is not an intervention in and of itself, but rather a component of an overall treatment strategy that looks at a number of risk factors and assists the drinker to develop strategies to minimise the risk of a return to risky or problem drinking.

Relapse prevention strategies can be used successfully with a variety of clients in different contexts, including residential and outpatient settings. The methods described in the model of relapse prevention developed by Marlatt and Gordon (1985) ${ }^{[141]}$ are separated into two types: specific intervention strategies and global self-control strategies.

\section{Specific intervention strategies}

The next step following assessment of risk involves teaching the drinker alternative coping responses that might be used in these situations. Dimeff and Marlatt (1995) noted that a number of areas may be covered, including cognitive restructuring, contracts to limit extent of use, reminder cards, relapse rehearsal, and stress management. ${ }^{[168]}$ As has been set out in sections that refer to these procedures, practice of coping responses should be performance-based, and personalised to the drinker. There is also some potential benefit from conducting the relapse prevention skills training in pairs allowing buddy support for the client. Role plays, modelling of new behaviours, video-assisted and direct feedback of performance will be valuable in developing skills for dealing with potential relapse situations.

Behavioural coping responses are: physical or some distracting activity, the consumption of food or non-alcoholic drink, escaping the situation, and relaxation procedures. Cognitive coping includes: thinking of the positive health consequences of not drinking and the negative health and other consequences of resuming risky drinking, and using thoughts related to delay or distraction. The range of methods used in training drinkers in these procedures includes mainly rehearsal, real or imagined, with evaluation and feedback of performance. Saunders and Allsop (1991) have developed these procedures further within the stages of change model, and the procedures they set out assist in developing a comprehensive relapse prevention approach. ${ }^{1699}$ Dimeff and Marlatt (1995) have provided a session-by-session guide to relapse prevention. ${ }^{[168]}$

The relapse prevention approach is also designed to deal with the lapse to drinking. The client's reaction to such lapses is viewed as crucial in determining whether drinking will continue or not. A single lapse can result in a complete return to drinking because the drinker sees the lapse as an indication of powerlessness over alcohol. ${ }^{[168]}$ The relapse prevention model teaches the client to view a lapse as a temporary return to drinking or risky drinking and not as a complete failure. Moreover, there is an emphasis on learning from the events preceding the lapse and making strategic plans for limiting future lapses.

\section{GLOBAL INTERVENTION STRATEGIES}

A final part of the approach is developing global intervention strategies where the client changes his or her lifestyle in ways which decrease the likelihood of drinking. The aim is to increase the client's capacity to cope effectively with pervasive stress factors. For example, therapy may focus on encouraging the client to take up recreational activities and behaviours that are incompatible with drinking alcohol, substituting indulgences, using coping imagery, and balancing perceived external demands (shoulds) with perceived desires (wants). [1683] 


\section{- Individual assessment of risk factors should be undertaken before identifying relapse prevention strategies. \\ - Relapse prevention should include immediate and global strategies that increase the client's ability to avoid relapse and to cope with more pervasive stressors. \\ - Clinicians should make arrangements for the client to continue contact after the relapse prevention program is complete. This can be done by phone, or in person. See Chapter 10: Extended care for more details.}

\section{Pharmacotherapies for relapse prevention}

Both naltrexone and acamprosate have been shown to improve treatment outcomes when added to other components of alcohol treatment. For clients who are motivated to take the medication, both are potential tools in preventing relapse to heavy or dependent drinking. For some, medication is associated with a critical period of sobriety, during which the client can learn to maintain abstinence without the help of medication.

Acamprosate is thought to reduce drinking by modulating the brain glutamate function which is implicated in withdrawal symptoms. Naltrexone is an anti-craving drug that reduces the chance of a lapse becoming a relapse. It acts upon the brain's opiate receptors. Both acamprosate and naltrexone have been approved for use as part of a comprehensive treatment plan for alcohol dependence.

The evidence for disulfiram is weaker. However, it is included here as one option for relapse prevention. There is some evidence that the combined use of psychosocial intervention, acamprosate and disulfiram is more effective in preventing relapse than one or the other medication alone. ${ }^{[170]}$ A head to head open label trial of acamprosate and naltrexone found that naltrexone was more effective than acamprosate in achieving abstinence, reducing craving, increasing the risk of and time to relapse, and reducing average drinks per drinking day. One randomised controlled trial suggests that combined naltrexone and acamprosate may be more effective than acamprosate alone, but no more effective than naltrexone alone. ${ }^{[17]]}$

\footnotetext{
Prescribers are referred to the MIIMS Annual for detailed information albout disulfiram, naltrexone and acamprosate (listed and marketed respectively as Antalbuse (r), Re Via (r) and Campral (r)), The product information should be reviewed before prescribing these medications.
}

\section{INTEGRATION WITH PSYCHOSOCIALTREATMENTS}

Pharmacotherapy for relapse prevention should always be accompanied by close follow-up by the prescribing doctor. Each client requires a comprehensive treatment plan, which includes dealing with psychosocial issues, as described in the previous section of this chapter.

Treatment is significantly more successfull when the client is receiving psychosocial treatment and is compliant with the medication, so psychosocial interventions should address this issue. Refenral to a specialist alcohol and drug counselling service may also be appropriate. 


\section{INCREASING MEDICATION COMPLIANCE}

Naltrexone has been shown to be more effective than placebo only among highly compliant participants ${ }^{[172]}$ Results from another trial suggest that the poor outcomes for naltrexone participants were owing to more adverse effects and hence, poor medication compliance. ${ }^{[173]}$

An analysis of data from two clinical trials showed that the gastrointestinal side effects of naltrexone - nausea, decreased appetite, dry mouth, vomiting, stomach pain, constipation and diarrhoea reduced medication compliance, but not treatment retention. ${ }^{[174]}$ Conversely, the neuropsychiatric effects - fatigue, dizziness, light-headedness, weakness, insomnia, sleepiness, confusions, blurred vision, headache, drowsiness and poor coordination- reduced treatment retention but not compliance.

Those more likely to experience moderate to severe nausea in response to naltrexone include:

- Lighter drinkers with shorter periods of abstinence

- Female clients

- Younger clients

There may be several other reasons that a patient is reluctant to comply with pharmacological treatment ${ }^{[175]}$ :

- For some, there is a stigma attached to taking medication for an alcohol use disorder. Many patients see taking medication as cheating and believe that they should have sufficient willpower to conquer the disorder unassisted. This belief may be reinforced by others in the patient's social network.

- Unlike medications that relieve distress, naltrexone blocks the reinforcing effects of alcohol. Although it probably reduces craving for alcohol, there is no inherent reward for complying with naltrexone or acamprosate.

- Related to this is that for some patients, these medications have unpleasant side effects. For these patients, not only is the medication inherently unrewarding, it is actually aversive. The commonest side effect is nausea, which usually abates after one or two weeks.

- Fears about the safety and side effects of the medication. Many patients probably do not know anything about the medication and may be quite fearful about taking it.

- Cost. In Australia, acamprosate and naltrexone are subsidised by the Pharmaceutical Benefits Scheme, provided the correct procedures are followed. However, patients may be reluctant to make co-payments and this should be clarified at the time of starting treatment.

Compliance therapy, based on cognitive-behavioural and motivational interviewing techniques, has demonstrated effectiveness with psychotic patients. ${ }^{[176]} \mathrm{A}$ pilot trial of compliance therapy with alcohol dependent patients being treated with acamprosate currently underway in Sydney, Australia has shown promising results. ${ }^{[177]}$ The six session intervention is outlined below:

- Sessions one and two: Elicit beliefs about problem and treatment. Review history, examine benefits of treatment.

- Sessions three and four: Explore ambivalence towards treatment, the pros and cons of treatment, direct and indirect benefits. Correct erroneous beliefs about medication, for instance side effects vs. symptoms.

- Sessions five and six: Highlight the need for treatment maintenance; enhance self-efficacy by 
focusing on achievements, frame medication and treatment as a choice that helps the client stay well to achieve goals, relapse prevention.

At the time of writing, the trial was incomplete. There was a trend towards longer time using acamprosate and longer time to relapse in the experimental group. A larger trial will start in 2003.

In a review of compliance therapy, a combination of educational/cognitive, behavioural and affective strategies was found to work better than any single-focus strategy: ${ }^{178]}$

- Educational/cognitive strategies were those designed to convey information through one-toone or group teaching, written and audiovisual materials, and telephone instructions.

- Behavioural interventions used targeting, modelling, contracting, packaging and dosage modifications, tailoring, rewards, reminders, skills building and practice activities, shaping, or reinforcing of specific behavioural patterns.

- Affective interventions used appeals to feelings and emotions or social relationships and supports, such as family support, counselling, and supportive home visits. Interventions targeting patients with mental health problems were successful across the board, with reminders and education delivering the most consistent improvements.

Another review suggests that the most important single intervention is recalling patients who miss appointments and making every effort to keep them in care. ${ }^{[179]}$

Compliance with pharmacotherapies may be assisted by:

- Eliciting the patient's thoughts and concerns about taking medication and using cognitive restructuring techniques to help them change unhelpful or maladaptive thoughts about taking medication.

- Providing the patient with a realistic view of the way in which the medication can help, its side effects, and any risks associated with its use. This includes education about pain relief for emergencies, that is, no opioid-based pain relief can be administered whilst the patient is using naltrexone.

- Using motivational interviewing techniques to help the patient to identify their personal costs and benefits of taking the medication.

- Providing the patient with some take-home reading material about the medication.

- Suggesting AA for patients with an abstinence goal.

- Tailoring the psychosocial intervention according to the patient's drinking goal: some studies show that coping skills training combined with naltrexone is better for helping patients cope with lapses and relapses, whereas supportive therapy is more effective in helping patients to maintain abstinence (see Chapter 8: Psychological Interventions for more information on these treatments).

- Following up clients who miss appointments. 


\section{ACAMPROSATE}

\section{Suitability for acamprosate}

There is little evidence to inform decisions about who is suitable for acamprosate. However, some evidence and clinical expertise suggests that the following patients may benefit: ${ }^{[175]}$

- Patients who are moderately to severely alcohol dependent and are medically stable

- Those who are willing to be in a collaborative relationship with a health care worker or a support group. It is a Pharmaceutical Benefits Scheme requirement that acamprosate is provided for "use within a comprehensive treatment program for alcohol dependence with the goal of maintaining abstinence".

- Patient's willingness to take and comply with acamprosate is an important factor in assessing suitability.

- Acamprosate is contraindicated in patients with a known hypersensitivity to the drug, renal insufficiency or severe hepatic failure (Childs Pugh classification C). ${ }^{\text {[9] }}$

- The safety of acamprosate in pregnancy or lactation has not been established so it should not be administered to women who are pregnant or breastfeeding. ${ }^{\text {[95] }}$

\section{Interaction with other drugs}

- Acamprosate does not interact with alcohol.

- There is some debate about whether tetracyclines are rendered inactivate by the calcium component in acamprosate during concurrent administration.

\section{Starting treatment}

- Acamprosate dosing is recommended to begin within one week of the patient's last drink (within one month is acceptable). ${ }^{[180]}$

- Acamprosate has been shown to be safe during detoxification. However, it is preferable to start acamprosate once withdrawal has settled so that withdrawal symptoms are not confused with medication side effects.

- Some clinicians do not prescribe acamprosate to patients who refuse to stop drinking alcohol. This is not because of drug interactions, but because they consider that patients who are unwilling to stop drinking lack the motivation to change their behaviour, hence, an adjunctive medication such as acamprosate or naltrexone would be a waste of time.

- Medical history should be taken, as per Chapter 3: Screening and assessment.

- Physical examination may include assessment for signs of chronic liver disease and hepatic failure. The assessment of hepatic insufficiency is done via clinical examination and liver function tests.

- Testing may include tests of kidney function (urea and electrolytes), since 90 percent of acamprosate is excreted through the kidney, and liver function tests, since it is contraindicated for patients with hepatic failure.

- Once assessment is complete, discuss treatment goals and plan with the patient

- Patient education should cover how the medication works, what side effects to expect, and realistic expectations about reductions in cravings. Explain that patients typically do not feel any different on treatment, and that the drug only reaches desired levels in the brain after one to two weeks.

- Arrange for a follow-up visit within one week, as early drop-out is common. 


\section{Dosage}

Acamprosate is formulated in tablets of $333 \mathrm{mg}$, with the recommended dose for adults being $1998 \mathrm{mg}$ (six tablets) orally in three divided doses, with meals. Adults under 60kg should take 1332 $\mathrm{mg} /$ day (four tablets/day in three doses: two, one and one).

\section{Ongoing treatment}

Treatment should continue even if the patient lapses; psychosocial relapse prevention techniques should be used to deal with the lapse or relapse.

\section{Adverse effects and their management}

Acamprosate is well tolerated and its predominantly gastrointestinal adverse effects, commonly diarrhoea, usually resolve spontaneously. Mild abdominal pain has been reported in four percent of acamprosate patients and two percent in placebo patients. Rash or isolated pruritus, parasthesiae, decreased (and sometimes increased) libido and confusion have all been reported at low frequencies.

The following strategies are recommended:

- Patient education about expected side effects and duration.

- To limit confusion between prolonged alcohol withdrawal symptoms and side effects of acamprosate, it is preferable to start acamprosate once withdrawal has settled.

\section{Treatment duration}

The usual treatment period is three to six months, but the decision on the duration of treatment should be made by the patient and the doctor on a case by case basis after discussion of both the positive and negative effects of the medication.

\section{Clinical considerations during treatment}

- Dealing with continued drinking (see earlier material in this chapter on psychosocial relapse prevention, and Chapter 8: Psychosocial interventions, specifically motivational interviewing and goal setting).

- Monitoring and attending to physical and mental health.

- Using acamprosate in conjunction with disulfiram is a possibility for patients who can be supervised and are motivated to abstain from alcohol.

- Medication compliance - some patients will have difficulty remembering to take pills three times per day. Reminder and reward systems may be necessary. See earlier material in this chapter on compliance therapy.

- Acamprosate therapy does not preclude participating in other treatment or support activities such as counselling, $\mathrm{AA}$, and/or other support networks.

\section{Ending acamprosate therapy}

At present there is no evidence of a withdrawal syndrome following the use of acamprosate or developing dependence on acamprosate, so patients should not experience withdrawal symptoms when they cease taking it. ${ }^{1800}$ Nevertheless, dose reductions may have psychological benefits for the patient. Psychosocial relapse prevention interventions should continue beyond the end of pharmacotherapy. 


\section{NALTREXONE}

Naltrexone is an anti-craving drug that reduces the chance of a lapse becoming a relapse. It acts on the brain's opiate receptors. It has been approved for use as part of a comprehensive treatment plan for alcohol dependence.

\section{Suitability for naltrexone}

There is little evidence to inform decisions about who is suitable for naltrexone. However, some evidence and clinical expertise suggests that the following points should be taken into account: [175]

- Patients who are moderately to severely alcohol dependent, medically stable, and who are not currently using opioids, may be suitable candidates. Narcan challenge is not required unless recently opioid dependent.

- Naltrexone is indicated for patients who experience strong cravings for alcohol after a priming dose. ${ }^{[180]}$

- The patient's willingness to comply with the medication should be assessed and discussed.

- Naltrexone is contraindicated for people with acute hepatitis or liver failure, or with a history of sensitivity to naltrexone.

- There are no well controlled studies of the safety of naltrexone during pregnancy or lactation. The safety of naltrexone with patients younger than 18 years old has not been established. ${ }^{\text {95] }}$

- Patients requiring opiate-based pain relief are not suitable candidates.

- One study found that naltrexone patients had worse outcomes on depression scores than placebo patients. ${ }^{[181]}$ This result should be interpreted with caution. However, given the high prevalence of depression among alcohol dependent patients, regular monitoring of depression using an instrument such as the Beck Depression Inventory is recommended. ${ }^{[6]}$

- Naltrexone may be more effective for preventing relapse to heavy or problem drinking than for maintaining abstinence from alcohol.

- In combination with naltrexone, coping skills therapy appears to be more effective than abstinence-oriented supportive therapy in reducing relapse risk and alcohol consumption. Coping skills therapy may help clients to cope with lapses. However, supportive therapy with naltrexone produces higher rates of abstinence.

\section{Interaction with other drugs}

- Naltrexone induces precipitated opiate withdrawal in patients who are currently opiate dependent. It is contraindicated in patients currently being maintained on methadone.

- Naltrexone does not appear to alter the absorption or metabolism of alcohol; however some patients have reported nausea from drinking alcohol while taking naltrexone.

- Caution should be taken with other drugs, as the interaction of naltrexone and most other medications has not been tested. It is not ordinarily recommended for use with disulfiram because both are potentially hepatotoxic. 


\section{Starting treatment}

- Treatment should begin after the symptoms of acute alcohol withdrawal have subsided, usually three to seven days after the patient's last drink.

- As for acamprosate, there is some debate about starting naltrexone while the patient is still drinking. The issue here is not drug interaction, but motivation for treatment.

- A medical history should be taken, as per Chapter 3: Screening and assessment.

- Physical examination may include assessment signs of chronic liver disease and hepatic failure. The assessment of hepatic insufficiency is done via clinical examination and liver function tests.

- Once assessment is complete, discuss treatment goals and plan with the patient.

- Patient education should cover how the medication works, what side effects to expect, realistic expectations about reductions in cravings.

- Arrange for a follow-up visit within one week, as early drop-out is common.

\section{Dosage}

Naltrexone is administered orally at $25 \mathrm{mg}$ for one to two days and then increased to the standard dose of $50 \mathrm{mg}$ daily. Naltrexone is available in $50 \mathrm{mg}$ tablets. Maintenance doses of less than $50 \mathrm{mg} /$ day may be considered for patients who do not tolerate to the standard dose. It may be preferable to reduce the dose to $25 \mathrm{mg} /$ day to avoid non-compliance because of adverse side effects. Some women may not need as high a dose as men; this should be monitored by the prescribing doctor.

Doses of up to $100 \mathrm{mg} /$ day have been used with patients who report persistent feelings of craving, discomfort, and brief relapses. Medical monitoring of such patients is critical. Intensifying other treatment components should be considered first and the reason for treatment failure explored. However, it would appear that some patients do metabolise naltrexone more quickly than others and therefore need a higher dose.

\section{Continuing treatment}

- There is some evidence to support the targeted use of naltrexone, i.e. using it only when cravings are severe, or at high-risk relapse times.

- Treatment should continue even if the patient lapses; psychosocial relapse prevention techniques should be used to deal with the lapse or relapse. 


\section{Adverse effects and their management}

Common adverse effects include nausea, headache, dizziness, fatigue, nervousness, insomnia, vomiting, and anxiety in about 10 percent of patients.

The following strategies are recommended:

1. Patient education about expected side effects and duration, reassurance that these effects are not serious and resolve with cessation of therapy.

2. Timing of doses: establishing a routine; ideally taken in the morning with food; or timed to prevent strongest cravings.

3. Splitting the dosage: half in the morning and half in the evening.

4. Management of nausea: patients should be advised to take naltrexone with food. Consider dose reduction, slow titration, and stopping the medication for three to four days before reintroducing it at a lower dose.

5. Gradual introduction of medication (see dosage information).

6. Distinguishing between prolonged alcohol withdrawal symptoms and side effects of naltrexone.

\section{Treatment duration}

The usual treatment period is three months, but six to twelve months may be necessary in some cases. This decision should be made on a case-by-case basis between the patient and the doctor, based on side effects, history of relapse, social and family circumstances, and other individual factors.

\section{Clinical considerations during treatment}

- Dealing with continued drinking: encourage continuation of medication, also see earlier material in this chapter on psychosocial relapse prevention, and Chapter 8: Psychosocial interventions, specifically motivational interviewing and goal setting.

- Monitoring and attending to physical and mental health as depression and dysphoria are recognised side effects of naltrexone.

\section{Ending naltrexone therapy}

Naltrexone does not appear to produce dependence so patients should not experience withdrawal symptoms when they cease taking it. ${ }^{1800}$ Patients can safely come off naltrexone straightaway. Even so, dose reductions may have psychological benefits for the patient. Psychosocial relapse prevention should continue beyond the end of pharmacotherapy. 


\section{TABLE 9.1: SUMMARY OF ACAM PROSATE AND NALTREXONE CHARACTERISTICS}

\begin{tabular}{|c|c|c|}
\hline Drug & Naltrexone & Acamprosate \\
\hline Recommendation & $\begin{array}{l}\text { First-line treatment for moderate to } \\
\text { severely dependent patients }\end{array}$ & $\begin{array}{l}\text { First-line treatment for moderate to } \\
\text { severely dependent patients }\end{array}$ \\
\hline Mechanism of action & Antagonism of opiate receptor & $\begin{array}{l}\text { Mechanism unknown (involves } \\
\text { restoration of normal activity in } \\
\text { glutamate and GABA systems) }\end{array}$ \\
\hline Reaction with alcohol & $\begin{array}{l}\text { - No aversive reaction } \\
\text { - Prevents pleasant "high" effect } \\
\text { of alcohol } \\
\text { - Sedation and adverse } \\
\text { psychomotor effects still occur }\end{array}$ & $\begin{array}{l}\text { No aversive reaction } \\
\text { No alteration of CNS effect of } \\
\text { alcohol or withdrawal symptoms } \\
\text { Evidence of reduction }\end{array}$ \\
\hline $\begin{array}{l}\text { in craving } \\
\text { Yes }\end{array}$ & & Yes \\
\hline $\begin{array}{l}\text { Evidence of reduction } \\
\text { in alcohol intake }\end{array}$ & Yes & Yes \\
\hline $\begin{array}{l}\text { Evidence of increased } \\
\text { abstinence }\end{array}$ & Yes & Yes \\
\hline $\begin{array}{l}\text { Evidence of effect post- } \\
\text { drug therapy }\end{array}$ & No & Yes \\
\hline Contraindications & $\begin{array}{l}\text { - Opiate dependence } \\
\text { - Chronic pain requiring treatment } \\
\text { with opiates } \\
\text { - Hepatic failure or active hepatitis } \\
\text { (ALT >3x normal) } \\
\text { - Renal impairment } \\
\text { - Pregnancy (ADEC B3) } \\
\text { - Lactation (safety not established) }\end{array}$ & $\begin{array}{l}\text { - Renal impairment (excreted } \\
\text { unchanged in the kidney) } \\
\text { Pregnancy (ADEC B2) } \\
\text { - Lactation (no data in humans) }\end{array}$ \\
\hline Drug interactions & $\begin{array}{l}\text { Opioids (antagonism of action) } \\
\text { Disulfram (combination may result } \\
\text { in increased hepatotoxicity) }\end{array}$ & $\begin{array}{l}\text { None with diazepam, disulfiram or } \\
\text { imipramine }\end{array}$ \\
\hline Adverse effects & $\begin{array}{l}\text { - Nausea* } \\
\text { - Headache* } \\
\text { - Dizziness } \\
\text { - Anxiety } \\
\text { - Fatigue } \\
\text { - Insomnia } \\
\text { - Somnolence } \\
\text { - Rarely hepatotoxicity }\end{array}$ & $\begin{array}{l}\text { - Diarrhoea* } \\
\text { - Nausea, vomiting } \\
\text { - Abdominal pain } \\
\text { - Rash, pruritis } \\
\text { - Rarely libido changes and } \\
\text { bullous skin reactions }\end{array}$ \\
\hline $\begin{array}{l}\text { Preparation before } \\
\text { commencing treatment }\end{array}$ & $\begin{array}{l}\text { - Start after acute alcohol } \\
\text { withdrawal but presence of } \\
\text { alcohol is not a contraindication } \\
\text { - Ensure not dependent on opiates }\end{array}$ & $\begin{array}{l}\text { Start after acute alcohol withdrawal } \\
\text { but presence of alcohol is not a } \\
\text { contraindication }\end{array}$ \\
\hline Dose & $\begin{array}{l}50 \mathrm{mg} \text { daily (can start on } 25 \mathrm{mg} \\
\text { for 2-3 days to reduce incidence of } \\
\text { side effects) }\end{array}$ & $\begin{array}{l}>60 \mathrm{~kg} 2 \text { tabs tds, } \\
\varangle 60 \mathrm{~kg} 2 \text { mane, } 1 \text { noon and } 1 \text { nocte }\end{array}$ \\
\hline Cost & $\begin{array}{l}\text { Provided at no cost to the client at } \\
\text { some specialist D\&A agencies. Can } \\
\text { also be obtained as an authority } \\
\text { script on the PBS. }\end{array}$ & $\begin{array}{l}\text { Obtained as an authority script on } \\
\text { the PBS. }\end{array}$ \\
\hline
\end{tabular}

*Common side effects

Reproduced with permission from Next Step Drug and Alcohol Services, Western Australia. Sim, 2002. 


\section{DISULFIRAM}

Disulfiram aims to assist the drinker to achieve and maintain abstinence by interacting with alcohol to create an unpleasant reaction. The research evidence indicates that maximal effect is achieved when the disulfiram is provided to the patient under supervision. Despite some evidence for its effectiveness, disulfiram is rarely used in practice. ${ }^{[1]}$ The drug is costly and is not readily available, although some pharmacists will order it in on receipt of a script. Disulfiram is a useful adjunct to treatment in the short term, provided that it is supervised to ensure that the patient takes an adequate dose regularly.

Disulfiram primarily works by inhibiting the action of enzymes (aldehyde dehydrogenase) involved in the metabolism of alcohol. The results of consuming alcohol while on disulfiram are unpleasant. They include flushing, dizziness, nausea and vomiting, irregular heart beat, breathlessness and headaches. Disulfiram acts as a deterrent to drinking because the patient expects to experience these negative consequences if they drink alcohol. ${ }^{[182]}$

\section{Eligibility for disulfiram}

Disulfiram is an appropriate medication for patients who:

- Are motivated to abstain from alcohol.

- Accept that there is a need for an external control on their drinking.

- Display no medical or psychosocial contraindications.

- Are prepared to be supervised in the daily dosing of the medication.

\section{Interaction with Other Drugs}

- As described above, disulfiram interacts with the metabolism of alcohol.

- Refer to MIMS for other interactions, that is, with isoniazid, phenytoin, some benzodiazepines, anticoagulants, metronidazole, paraldehyde. ${ }^{[95]}$

\section{Starting Treatment}

- Discuss treatment goals and plan with the patient.

- Treatment should begin after detoxification. At a minimum, the patient should abstain from alcohol at least 24 hours before taking disulfiram.

- Medical history should be taken as per Chapter 3: Screening and assessment

- Patient education is critical. Discussion of the effects of the drug when alcohol is taken is an important part of the therapeutic strategy, as the patient's anticipation of its effects will greatly enhance the drug's effectiveness as a deterrent against drinking.

- Discuss motivation and supervision with the patient. The patient needs to understand supervised disulfiram as a useful strategy to assist in the maintenance of the choice of abstinence. In that way, the patient sees disulfiram as an aid that assists but does not detract from the patient's own responsibility and choice in maintaining abstinence. 


\section{Dosage}

A dose of 200 to $400 \mathrm{mg}$ of disulfiram per day is appropriate (tablets are $200 \mathrm{mg}$ each), although some commentators have suggested that much higher doses are required before a reaction to alcohol ingestion can be assured. ${ }^{1833}$ Although there is a risk that this dosage regimen is insufficient to guarantee a reaction upon drinking in some people, dosages above this level expose the patient to possible side effects. An aversive reaction to the drug can be tested for by prescribing a dose of $400 \mathrm{mg}$ and then reducing to $200 \mathrm{mg}$ according to the patient's response.

\section{Continuing treatment}

Disulfiram is likely to be a useful treatment for the first three to six months of treatment. After that time its use should be discontinued and the patient should be encouraged to maintain abstinence without disulfiram.

Ongoing treatment should include psychosocial relapse prevention strategies. Treatment should be continued even if the patient relapses.

\section{Supervision}

Supervision of disulfiram dosing is likely to have a marked effect on compliance, and to greatly improve the effectiveness of this intervention. Supervision can present problems for some patients. It might be difficult to organise: the patient needs to be prepared for public disclosure either with the clinician or some other person who is chosen to supervise.

The patient's spouse/partner is an obvious choice for married/de facto patients, and can play a valuable role in treatment. However, the non-alcohol dependent partner might feel the disulfiram contract places the responsibility for the drinking (inappropriately) on their shoulders. He or she has probably learned that they cannot control the partner's drinking. It is therefore important to stress to the patient's partner that:

- The spouse cannot be expected to control the other person's drinking, and emphasise that their responsibility as supervisor is simply to observe the partner taking the disulfiram.

- The supervision should not be viewed as a coercive checking-up operation.

\section{Summary}

Oral disulfiram is not recommended as a first-line strategy. It may be an appropriate short term strategy when the clinician and patient agree that there is a need for some form of external control over drinking behaviour to be present, and provided that the patient's compliance with dosing is adequately supervised either by a spouse or a health professional involved in treatment. Disulfiram is, however, difficult to obtain in Australia. 
Psychosocial relapse prevention strategies are effective in reducing alcohol intake, reducing the severity of relapse, and improving psychosocial outcomes.

Relapse prevention works equally well across treatment settings, in II combination with different types of treatment, and in group or individual sessions.

The optimum duration and intensity of relapse prevention is unclear, and may vary from client to client with different problems and needs.

Acamprosate is moderately effective as an adjunctive therapy in reducing relapse risk in the short term.

Acamprosate appears to be moderately effective as an adjunctive therapy in the longer term (up to two years).

Acamprosate appears to be safe for use during alcohol withdrawal, that is, no interactions were observed with withdrawal medications.

Acamprosate therapy should be started within one week of completing withdrawal.

One trial found acamprosate to be equally effective imespective of compulsory participation in counselling.

Clients who receive treatments that include strategies to increase compliance have better outcomes.

Naltrexone as an adjunctive therapy is moderately effective in reducing relapse risk and alcohol consumption in the short term.

Naltrexone may be more effective for preventing relapse to heavy or problem drinking than for maintaining abstinence from alcohol.

Targeted naltrexone in response to cravings may be of some use in reducing relapse risk.

In combination with naltrexone, coping skills therapy appears to be

II

more effective than abstinence-oriented supportive therapy in reducing relapse risk and alcohol consumption. Coping skills therapy may help clients to cope with lapses. However, supportive therapy with naltrexone may produce higher rates of abstinence.

One trial found naltrexone without compulsory psychosocial intervention to be effective in reducing relapse. more effective than acamprosate but not naltrexone. However, outcomes for naltrexone and acamprosate patients were not significantly different.

One pilot trial with acamprosate patients and evidence from other areas of mental health suggests that an intervention based on motivational interviewing and cognitive-behavioural strategies can increase medication compliance and lead to better treatment outcomes. 
Psychosocial relapse prevention strategies are Strong recommended for use with all moderate to severely alcohol dependent clients.

Psychosocial relapse prevention is best delivered after acute withdrawal symptoms have subsided.

Identify high risk situations. Assess for risk factors: cravings, disease beliefs, coping skills.

Assessment for relapse prevention suitability should take into account comorbid disorders, current coping skills, social skills, cognitive impairment, other drug use

Acamprosate is recommended as an adjunct to psychosocial relapse prevention for moderate to severely alcohol dependent clients, with the exception of those contraindicated as per table 9.1.

Naltrexone is recommended as an adjunct to psychosocial relapse prevention for moderate to severely alcohol dependent clients, with the exception of those contraindicated as per Table 9.1.

Patients who are opioid dependent should not be prescribed naltrexone. All patients should be warned of the potential for opioid withdrawal syndrome if any form of opiates is taken, that is, heroin, methadone, and opioid analgesics.

For some patients, general practitioner management to accompany acamprosate and naltrexone will be sufficient. Others (e.g. chronic relapsers) will need more intensive psychosocial interventions. Thus, psychosocial treatment should be tailored to the needs of the patient and should include relapse prevention strategies.

Acamprosate and naltrexone should be started within one week of detoxification.

Acamprosate and naltrexone are usually taken for three to six months, and in some cases up to 12 months. Optimum treatment duration has not been established.

Many patients find it difficult to comply with a Strong

Moderate

Strong

Strong medication regimen. This issue should be addressed with compliance therapy which is based on motivational interviewing and cognitive-behavioural strategies. 
Chapter 10: Extended care 


\section{Chapter 10: Extended care}

\section{CHAPTER AIM}

The aim of this chapter is to describe:

- The range of extended care strategies available for patients with an alcohol use disorder (abuse or dependence).

- The Alcoholics Anonymous (AA) approach and how AA participation might fit with other interventions for alcohol problems.

Successful treatment of any condition which is subject to relapse, such as alcohol dependence, often requires ongoing and extended assistance. The key issues include retention in treatment, assertive outreach, and effective referral. AA is included as an aftercare strategy because there is some evidence suggestive of its suitability for this purpose. However, many patients choose to attend, and may benefit from, AA sessions before or during the initial treatment, as well as using the self-help group to maintain sobriety post-treatment.

\section{Treatment retention}

The chronic nature of alcohol dependence means that treatment is often long term. The factors that retain clients in treatment include:

- Client variables (pre-treatment motivation and therapeutic involvement, see chapters three and eight).

- Treatment variables (strength of the therapeutic relationship, perceived helpfulness of the treatment services, empathy of the clinician, removal of practical bamiers such as transportation, and the inclusion of relapse prevention training). ${ }^{[3,}{ }^{39]}$ Clinician characteristics and the therapeutic relationship are crucial to engaging the client in treatment (see Chapter 8: Psychosocial interventions). ${ }^{\text {[3] }}$

\section{Alcoholics Anonymous}

AA is the mainstay of the self-help approach to alcohol problems in Australia, although there are other group approaches. It is the prototype of the self-help approach to assisting people with alcohol problems, and is the major organisation available of this kind. AA was formed in the USA in 1935 and came to Australia in 1945. There are now approximately 1700 AA groups in Australia. Currently, women make up about 35 percent of the membership 5 .

The essence of the self-help group approach to alcohol problems is that change is not facilitated or mediated by professionals, but is the result of the group members' own initiatives and support of each other. Because of the essential nature of self-help initiatives this form of intervention is not conceptualised as a form of treatment. It is a self-help organisation that assists people in working together to maintain abstinence from alcohol. Its wide availability and prominence in the community offers continuing support and assistance outside formal treatment. 


\section{HOW EFFECTIVE IS AA?}

The research into the effectiveness of conventional AA meetings suggests that it has a place as an adjunct to formal treatment, since participation predicts more positive long term outcomes for many clients. However, there are serious limitations to the research that has been conducted. First, few randomised controlled trials exist. Other limitations of the research are that many studies have lacked the statistical power to detect differences in treatment outcomes, many have used only drinking outcomes, and participation in AA meetings is an inherently heterogeneous experience. ${ }^{[184]}$ That is, AA operates differently even within a single region, so it is difficult to generalise about its processes.

\section{HOW AND FOR WHOM DOES IT WORK?}

AA participation has been shown to predict higher rates of abstinence post-treatment. Participants with more severe symptomatology are more likely to participate in AA. The practice of AA activities may lead to increased self-efficacy to abstain from alcohol. ${ }^{[133]}$

Given the poor outcome of participants coerced into AA participation, it would appear that mandating AA as a treatment is counterproductive. ${ }^{[185]}$ Individuals with higher network support for drinking may benefit most from AA participation. ${ }^{[186]}$ This is also the case when AA is used an aftercare strategy. ${ }^{[143,186]}$ The extent of active participation in AA processes may also predict better outcomes. ${ }^{[187]}$

\section{HOW SHOULD IT BE USED?}

Although these results provide limited evidence of the value of AA, they do suggest that AA assists in the reduction of alcohol problems at least for those clients who choose to attend the meetings. In summary, attendance at AA meetings is useful as an adjunct to therapy, and as an aftercare strategy for relapse prevention, particularly for clients with high network support for drinking. Table 10.1 outlines the 12 steps used in AA.

\section{TABLE 10.1:THE 12 STEPS OF ALCOHOLICS ANONYMOUS}

We:

1. Admitted we were powerless over alcohol and that our lives had become unmanageable.

2. Came to believe that a power greater than ourselves could restore us to sanity.

3. Made a decision to tum our will and our lives over to the care of God as we understood Him.

4. Made a searching and fearless moral inventory of ourselves.

5. Admitted to God, to ourselves, and to another human being the exact nature of our wrongs.

6. Were entirely ready to have God remove all these defects of character.

7. Humbly asked Him to remove our shortcomings.

8. Made a list of all persons we had harmed, and became willing to make amends to them all.

9. Made direct amends to such people wherever possible, except when to do so would injure them or others.

10. Continued to take a personal inventory and when we were wrong promptly admitted it.

11. Sought through prayer and meditation to improve our conscious contact with God as we understood Him, praying only for knowledge of His will for us and the power to cary that out.

12. Having had a spiritual awakening as the result of these steps, we tried to carry this message to alcoholics, and to practise these principles in all our affairs. 
Awareness of local AA activity, contact with the AA general service officer, familiarity with AA literature and attendance of some open AA meetings assist counsellors at times of referral. In particular, counsellors should be acquainted with a range of AA sponsors, so they can help clients meet others with similar interests. This process reassures the client that like-minded people attend AA. There are also AA groups that accommodate special needs, such as all-female meetings or meetings for homosexual members. AA is most appropriate for clients with alcohol dependence, who wish to work towards abstinence. Because it takes time to understand the full benefits of AA, these clients should be encouraged to attend AA meetings at least three times before making a decision about continued involvement.

Since AA is not viewed as a treatment it is not a sufficient intervention for alcohol problems. As noted earlier AA does not see itself as a treatment, and it fully acknowledges the need for drinkers to access professional assistance when required. Yet AA is available without charge to support changes in drinking to ex-drinkers, in many locations throughout Australia, and it will continue to be available after time-limited interventions have been terminated.

There is a risk that those with serious problems that are not dealt with within the self-help group will continue to suffer problems that could be ameliorated though professional assistance. The AA literature recognises this limitation, and states clearly the need to access the assistance of health professionals for serious problems that cannot be addressed by the self-help group. It is important for those on both sides of the debate to recognise the danger of making decisions or advising others on the basis of personal bias.

\section{Assertive outreach and structured aftercare}

Conventionally, aftercare is thought of as continuing contact between the client and clinician, once the initial treatment phase is completed. This can be "booster sessions" to maintain skills learnt in treatment, telephone contact initiated by the patient or clinician, or support and monitoring of progress as the drinker reintegrates into the community. Living without risky drinking may present unexpected problems that the patient needs assistance to deal with. This assistance should be a part of the overall intervention, and not seen as an optional "add-on" either by the patient or by the clinician. The patient should understand the importance of continued contact with the clinician and should be made to feel that contact is not only acceptable, but is also expected. It is too easy for a patient to feel unable to recontact, especially if a lapse occurs. Yet this is the critical time that requires assistance be available.

\section{CONTENT AND FORMAT OF AFTERCARE}

A highly structured approach to scheduling aftercare increases the likelihood of maintaining abstinence. [188]. In this study, aftercare consisted of individual problem-oriented treatment sessions. Patients had aftercare sessions scheduled on a calendar, which they were instructed to display prominently. They were instructed to attend aftercare regardless of drinking status and to reschedule missed appointments. A structured aftercare program is more effective than assistance on request. ${ }^{[1899],}{ }^{[190]}$ The structured approach in this instance significantly reduced the risk of relapse, decreased self-reported crime, and assisted unemployed persons to find employment.

Group aftercare is probably as effective as individual delivery. The content of aftercare depends 
upon the type of intervention used initially if the follow-up is to take the form of booster sessions, or it is determined by the problems and issues that arise for the patient within the post-intervention period. If the aftercare is run on an individual basis there is room for tailored problem solving approaches, and this has advantages. Run on a group basis, aftercare is less tailored but allows patients to form important support networks and to learn from each other's mistakes and successes. Relapse prevention strategies can form an important part of this aftercare and it allows lapses to be dealt with without becoming relapses. Self-help can be integrated, but it is recommended that there be a structure to the aftercare just as there needs to be to the intervention. The mix of selfhelp and structured professionally run aftercare in the McAuliffe and Ch'ien (1986) study provides a useful model for those agencies that wish to incorporate both a self-help and a relapse prevention approach. ${ }^{[190]}$

\section{SUMMARY OF EVIDENCE: EXTENDED CARE}

LEVEL OF EVIDENCE

Factors involved in long term recovery include treatment retention, III-1 social support networks, and assertive aftercare and follow-up.

Assertive, structured aftercare is more effective in reducing relapse than aftercare on request.

Evidence from natural recovery studies highlights the importance of social support and an intact sense of self-worth in maintaining controlled drinking or abstinence.

Voluntary participation in AA meetings may assist clients to III-1 maintain abstinence or reduce alcohol intake.

Coenced attendance at AA meetings is likely to be counterproductive.

Those with higier network support for drinking may benefit most III-1 from AA participation.

Attention should be given to the client's retention in treatment Strong and social support networks.

Aftercare should be structured and assertive, with follow-up of missed appointments.

Clients who show signs of dependence on alcohol should be made aware of the service offered by meetings of Alcoholics Anonymous (AA). Mandated AA participation is not recommended.

Conventional AA meetings should not be viewed as treatment for alcohol dependence.

Moderate 
Chapter 11: Interventions for specific client groups. 


\section{Chapter 11: Interventions for specific client groups}

\section{CHAPTER AIM}

The aim of this chapter is to highlight specific treatment needs of

- adolescents and young adults

- clients with cognitive impairment.

- clients with comorbid disorders

- men and women

- Indigenous clients

Although the interventions described above are recommended for all clients - taking into account the severity of the alcohol problem, the treatment goal, and other indications from the assessment - certain groups have specific needs that should be addressed in the treatment program. The following notes, therefore, are in addition to the general recommendations.

\section{Adolescents and young adults}

The adolescent years are a period for experimentation, socialisation and engaging in high-risk substance use behaviours. ${ }^{[191]}$ Alcohol consumption is now occurring earlier in adolescence. Binge drinking and deliberate drinking to become intoxicated is common, with 11.7 percent of those aged 14 to 19 years and 14.7 percent of those aged 20 to 29 years drinking at risky or high risk levels. ${ }^{[6]}$

\section{ASSESSMENT}

The DSM-IV criteria for alcohol use disorders may have limitations when used with adolescents. ${ }^{[192]}$ Some symptoms have a very low prevalence in adolescents, that is, withdrawal and alcohol-related medical problems, which generally emerge only after several years of heavy drinking. Other symptoms such as hazardous use and alcohol-related legal problems are highly correlated to male gender, increased age and symptoms of conduct disorder. Some symptoms such as tolerance to alcohol may have low specificity for adolescents.

DSM-IV alcohol symptoms have been found to develop in three distinct stages among adolescents, with some dependence symptoms developing before some abuse symptoms. ${ }^{[193]}$ During the first stage, which generally occurs after three to four years of exposure to alcohol, adolescents typically develop three dependence symptoms: tolerance, drinking greater amounts or for a longer period of time than intended and spent more using alcohol; and two abuse symptoms: role obligation problems and social problems.

The second stage is characterised by three dependence symptoms: unsuccessful attempts to quit or cut down on drinking, reduced activities because of alcohol use and continued use despite physical or psychological problems; and three abuse symptoms: blackouts, hazardous use and alcohol-related legal problems. This stage of adolescent alcohol symptoms is often described as "psychological dependence". 
The third stage is characterised by the dependence symptoms of withdrawal. This stage is often referred to as "physiological dependence" of the adolescent alcohol symptoms.

These findings suggest that a flexible approach to diagnosis and treatment is needed with adolescent drinkers. Whilst some adolescents may not meet strict diagnostic criteria for an alcohol use disorder, this should not be a barrier to treatment.

The differences between adult and adolescent problem drinkers may have important implications for treatment. ${ }^{[194]}$ These differences include shorter drinking histories, more polydrug use, greater binge drinking, rapid social and physical changes and a wider range of co-existing life problems such as homelessness, poor performance at school, difficult parental relationships and low employment prospects, and a familial history of heavy alcohol and drug use. ${ }^{[194,195]}$

For information about assessment methods and instruments, refer to Chapter 3: Screening and assessment.

\section{ENGAGING ADOLESCENTS INTREATMENT}

Engaging adolescents in treatment is a critical issue, given their low treatment retention rates. The Clinical Practice Guidelines: Depression in Young People ${ }^{[196]}$ recommend a number of useful strategies for clinicians to engage adolescents in treatment. ${ }^{6}$

\section{TREATMENT}

In comparisons of treatment settings and types for adolescents, few differences in outcome have been found. ${ }^{[194,197,198]}$ One study evaluating inpatient and outpatient treatment found that better outcomes were obtained with longer treatment retention, although a short stay in treatment was better than no treatment at all. ${ }^{[194]}$

Research with adolescents for substance use and other disorders, , mood disorders suggests that cognitive behavioural therapy (CBT) may be the treatment of choice. Relaxation therapy, therapeutic support groups, social skills training, interpersonal therapy, and family therapy may also be effective. [196]

Brief and motivational interventions appear to be effective for adolescent heavy or binge drinkers, and have been useful in emergency department, high school, and college settings.

One review of an Australian adolescent residential treatment facility found that more than 50 percent of female clients of an adolescent drug and alcohol treatment facility have experienced sexual abuse. Seventy five percent of admitted clients had either attempted or contemplated taking their own life. Thirty percent were living on the street, and 18 percent in a refuge, and most of their clients had left school in or before year nine. ${ }^{195]}$ There may be comorbid mental health disorders that require attention, and assessment should be especially careful to detect these problems. Risky drinking may interfere with the developmental life experiences of the young person, and therefore treatment is also aimed at teaching appropriate skills and compensating for skills deficits. 
The implications of this include:

- Suicide risk should be assessed in all adolescent clients presenting with alcohol-related problems or signs of depression

- Adolescent drinkers may be more chaotic than many adult drinkers. Therefore harm reduction, outreach and crisis intervention will be a priority. Office-based therapy is not always be appropriate.

- Family members should be included in some sessions if possible, to deal with family/relationship issues.

- Brief interventions suit some adolescent drinkers who are in the early stages of their drinking pathway, and are a critical part of reducing the risk of ongoing alcohol problems. However, many young people will do better by forming ongoing relationships with counsellors. They may need to be encouraged to return when they want to or need to.

- A developmental approach of tolerance and acceptance accompanied by clear boundaries, is appropriate for many adolescent clients.

- Polydrug use is the norm among adolescent problem drinkers so other drug use should be assessed, and where necessary, addressed.

- Problem-solving, social, coping, vocational, educational and other life skills training may be helpful in preventing relapse once the client has stabilised. 
SUMMARY OF EVIDENCE: ADOLESCENTS AND YOUNG ADULTS

Many adolescents do not fit DSM-IV criteria for alcohol use disorders, even though they may still be experiencing negative physical, social, educational and vocational consequences because of alcohol.

There is no evidence to suggest that any one form of treatment or treatment setting is superior for adolescents with alcohol problems. However, research in other fields suggests that CBT may be the treatment of choice.

Brief and motivational interventions help to reduce alcohol consumption among adolescent heavy or binge drinkers, across a range of treatment settings.
LEVEL OF EVIDENCE

III-1

IV

II

\section{RECOMMENDATIONS:ADOLESCENTS ANDYOUNG ADULTS}

Given the limitations of DSM-IV criteria for alcohol use disorders with adolescents, a flexible approach to diagnosis and treatment is needed. Whilst some adolescents may not meet strict diagnostic criteria for alcohol dependence or abuse, this should not be a barrier to treatment.

Clinicians should focus on building and maintaining a relationship with adolescent clients through outreach, crisis intervention and harm reduction activities, and the use of an empathetic, non-judgemental, developmental approach to counselling.

Brief and motivational interventions help to reduce alcohol consumption among adolescent heavy or binge drinkers, across a range of settings.

Younger drinkers who require more intensive intervention may have different treatment needs to older drinkers. These needs should start to be identified during the assessment phase, and may include crisis intervention, assertive outreach, and building social support networks.

The recommended psychological treatment is CBT. Other potentially useful treatments include social skills training, family therapy, therapeutic support groups, and interpersonal therapy.

\section{STRENGTH OF} RECOMMENDATIONS

Strong

Strong

Strong

Strong
Moderate 


\section{Cognitive impairment}

Although the examination of the effect of cognitive functioning on treatment outcome is in its early stages, several recommendations can be made. ${ }^{[199]}$ Many studies suggest that impaired cognitive functioning is related to poorer treatment outcome, particularly for treatments that require the acquisition of new skills. Thus, a brief assessment of cognitive functioning should be an integral part of the assessment procedure and results should be used to guide treatment planning. ${ }^{[200]}$

If significant impairment is suspected, a more thorough assessment by an appropriately qualified professional is indicated. Where severe cognitive impairment is present, treatment in an inpatient facility may be more effective than outpatient treatment. ${ }^{[107]}$ It should be noted that impairment can improve once drinking is stopped.

\section{- Screening for cognitive dleficits should be an integral part of the assessment procedure. \\ - Where cognitive impairment is confirmed, treatment elements that require heavy cognitive processing should not be employed as they are likely to be ineffective. ${ }^{[199]}$ \\ - Information presented to patients should be concrete and patients should be given opportunities to practise behaviours taught.}

Although it has been recognised for some time that many persons who suffer from risky drinking and alcohol dependence also suffer from cognitive impairment, there is little evidence on which treatments are most effective. Nevertheless, level of cognitive functioning should be used to guide treatment planning. Even subtle cognitive deficits could affect how treatment effectiveness in a number of ways.

Firstly, people who suffer from alcohol abuse or dependence may have difficulty processing all the relevant information about their problem and may be inflexible about changing behaviour. [199] It is important that the clinician realises that this inflexibility results from an inability to understand the need to change, rather than denial of a problem and refusal to change behaviour. In these situations, particularly where cognitive deficits are temporary, different treatment approaches may be used to engage the person in treatment.

\section{ENGAGINGTHE CLIENT INTREATMENT}

Many of the following strategies apply to all clients. However, they may be particularly important for engaging patients who suffer from cognitive deficits.

The following strategies may increase the client's engagement in treatment:

- Providing information to the client about treatment

The client may be more likely to enter treatment if they understand what treatment will involve, the process of treatment, and what they will be required to do.

- Discussing different treatment options with simple explanations

Although clients suffering from cognitive impairments should only be offered treatment interventions, which do not require complex cognitive processing and keep information and tasks concrete, the different options available to them should be discussed. Where the clinician judges that the client is capable of making a decision, the client should be involved in deciding which treatment to partake in.

- Establishing a positive relationship with the client by: 
$\triangleright \quad$ Keeping information simple and structured without being patronising.

$\triangleright \quad$ Adopting an empathetic, non-judgemental, and non-authoritarian approach.

$\triangleright$ Listening carefully to what the client has to say.

$\triangleright \quad$ Scheduling sufficient time for consultations.

$\triangleright \quad$ Treating clients with respect.

$\triangleright \quad$ Respecting confidentiality except where there is a threat to life or in cases of abuse.

- Maintaining contact

Maintaining contact with clients with cognitive deficits is very important. To increase the likelihood that clients attend appointments, clinicians should:

$\triangleright \quad$ Ring before a consultation to remind the patient they have an appointment.

$\triangleright \quad$ Keep the appointment at the same time on the same day to decrease the likelihood forgetting.

$\triangleright \quad$ Ring if an appointment has been forgotten and arrange an alternative time.

$\triangleright \quad$ Refer the patient to aftercare once treatment has been completed.

Cognitive deficits can also affect treatment by limiting the patients ability to effectively express their thoughts and feelings and to understand communication from the clinician. ${ }^{[199]}$ The clinician should keep all communication as simple as possible, and repeat information several times. The clinician should frequently check:

- That the client understands what is being said by the clinician.

- That the clinician understands what the client is saying.

Cognitive impairment can impair motivation, attention span, the capacity to evaluate situations critically and the ability to acquire new skills, but they can and often do improve with a period of abstinence from alcohol. ${ }^{[199]}$ Therefore, the possibility of improvement in cognitive functioning should be taken into account by allowing a sufficient period of abstinence from alcohol to elapse before finalising treatment planning. Interventions during the early stages of treatment may need to be kept relatively simple until or unless it is apparent that the client can cope with more complex information. Where cognitive impairment is apparent, treatment elements that require heavy cognitive processing should not be used as they are likely to be ineffective. ${ }^{2000}$ Information should be concrete and clients should be given opportunities to practise behaviours taught.

There is some evidence that cognitive impairment is associated with an increased risk of relapse. ${ }^{2000,201]}$ To avoid the chance of relapse, cognitively impaired patients should participate in psychosocial relapse prevention or be prescribed a pharmacotherapy (see Chapter 9: Relapse prevention for details). 


\section{SUMMARY}

Although evidence about the effect of cognitive deficits on treatment outcome is limited, available research indicates that treatment outcome may be adversely affected by cognitive impairment. Cognitive deficits are associated with an increased risk of relapse. There is less evidence about which treatments are more or less effective. However, one viable explanation is that poor treatment outcome is because of an inability of cognitively impaired patients to process the information imparted in therapy. Therefore, treatments that are simple, structured, and require less cognitive processing are thought to be more effective for patients with cognitive deficits.

\section{SUMMMARY OF EVIDENCE: COGITIIVE IMPAIRMENT}

Cognitive impairment adversely affects treatment outcome.

Cognitive impairment is associated with an increased risk of relapse.

Cognitively impaired patients have better outcomes with treatments that require little cognitive processing, are simple and well structured.

\section{LEVEL OF EVIDENCE}

III-2

III-2

III-2

A brief assessment of cognitive functioning should be a routine part

of assessment upon treatment entry.

More detailed assessment should be caried out where brief intervention suggests that a patient suffers from significant cognitive deficits.

Where cognitive impairment is confirmed, information presented to patients should be concrete and patients should be given opportunities to practise behaviours taught treatment.

Cognitively impaired patients should be engaged in treatment by the clinician by:

- providing information about treatment.

- discussing different treatment options.

- establishing a positive relationship.

- maintaining contact with the client.

The clinician should frequently check that the client understands what is being said, and that the clinician understands what the client is saying.

The possibility of improvement in cognitive functioning should be Moderate taken into account by allowing a sufficient period of abstinence from alcohol to elapse before finalising treatment planning. 


\section{Comorbid disorders}

Little controlled research has been conducted to evaluate the effectiveness of treatment for comorbid clients, despite the fact that a sizeable proportion of alcohol dependent clients have a comorbid mental disonder. In total, six percent of the National Survey of Mental Health and Well Being sample met the criteria for an alcohol use disorder. Just under two percent (1.9 percent) met the criteria for alcohol abuse, and 4.1 percent met the criteria for alcohol dependence. Of this group about one in five (20 percent) met the criteria for an anxiety disorder and nearly one in four (24 percent) met the criteria for an affective disorder (mood disorder). Other disorders associated with alcohol dependence include other substance use disorders, and psychosis. ${ }^{[202]}$

\section{ASSESSMENT}

Given the high prevalence of other mental disorders among patients with an alcohol use disorder, it is essential that checking for particularly common problems such as anxiety and depression symptoms is a routine part of the assessment. The Beck Depression Inventory and the Beck Anxiety Inventory are short but useful instruments for this purpose. ${ }^{[67,68]} \mathrm{The}$ Kessler 10 Symptom Scale is a scale of psychological distress, suitable for use as an outcome measure in people with anxiety and depressive disorders. It is likely to become the standard scale for use by general practitioners and mental health workers, since its use does not require the practitioner to have specific qualifications.

The AUDIT appears to be a suitable screening tool for identifying risky, problem and dependent alcohol consumption among psychiatric patients. ${ }^{\text {[2033 }}$ Assessment for comorbidity is covered in more detail in Chapter 3: Screening and assessment.

\section{PSYCHOSOCIAL INTERVENTIONS}

Several treatment issues arise with comorbid disorders. Should treatment be integrated, parallel or adjunctive? If the latter, which disorder should be treated first? What combination of therapies should be used? What implications does the order of onset have for treatment? The research to date, while providing some clues, does not adequately answer these questions.

Cognitive behavioural therapy (CBT) appears to be more beneficial than general counselling for drinkers with comorbid depression. ${ }^{[204]}$ Project MATCH data suggests that CBT was more effective than 12-step facilitation (TSF) for female, alcohol dependent patients with comorbid social phobia. For socially phobic males, there was a trend towards better outcomes in the TSF group. ${ }^{205]}$

Integrating motivational interviewing, CBT and family intervention with routine psychiatric care produced greater benefits for patients with comorbid schizophrenia and substance use disorders than routine psychiatric care alone. ${ }^{[206]}$ At 12-month follow-up, the integrated treatment group had better general functioning, a reduction in positive symptoms, and an increase in the percentage of days abstinent from alcohol or drugs.

Patients with a serious mental illness and a substance use disorder who received an integrated mental health and substance use treatment program had superior outcomes to patients assigned to a standard hospital treatment program. ${ }^{[207]}$

Differentiating between primary and secondary depressive disorders may have implications for 
treatment strategies, since secondary depression often abates once the alcohol use disorder is addressed. ${ }^{[208]}$ Almost 42 percent of dependent drinkers in Schuckit's study met criteria for a diagnosis of a major depressive episode at some time during their alcohol dependence. Of those, more than 60 percent reported a substance-induced depressive episode. Those with primary depression had a higher prevalence of independent depressive disorders in first-degree relatives. They were also more likely to be married, Caucasian, and female, or if male, to have a stable marital history. They typically had experience with fewer drugs and less treatment for alcohol problems, and were more likely to have attempted suicide. However, the clinical presentation of symptoms did not differ substantially between substance induced and primary depressive disorders, so determining the order of onset may be the best way to distinguish between primary and secondary depression.

According to Kavanagh, "Effective treatments focus on developing and maintaining motivation and promote re-entry after lapses. Studies with stronger effects tend to have assertive case management and (in the relatively chronic or severely affected populations usually studied) they extend over several months." (p. 63, ${ }^{[209)}$

\section{PHARMACOTHERAPIES}

There is no sound evidence for the capacity of SSRIs to reduce alcohol intake in the longer term. Although they do not seem to reduce alcohol consumption, ${ }^{[210,211]}$ they have been effective in reducing depression scores in alcohol dependent patients. ${ }^{[212]}$

Only one trial to date has specifically examined the effectiveness of SSRIs with comorbid patients. Cornelius et al (1997) administered fluoxetine or placebo to a randomised group of 51 alcohol dependent patients diagnosed with major depressive disorder, over a 12-week period, in an inpatient setting. ${ }^{[213]}$ Depression and alcohol consumption ratings were collected weekly during the 12-week period. Both depressive symptoms and total alcohol consumption over the trial were significantly lower in the fluoxetine group than in the placebo group. However, other studies have found either little effect on alcohol consumption, or that the effect dissipates quickly.

Buspirone, an anxiolytic, has been tested in one controlled trial with anxious alcohol dependent outpatients with some success. Buspirone patients were more likely to remain in treatment for the 12 weeks, had reduced anxiety, a slower return to heavy alcohol consumption, and fewer drinking days during the follow-up period. ${ }^{[214]}$

A controlled trial with desipramine (a tricyclic antidepressant) showed reduced relapse in alcohol dependent patients diagnosed with major depression, but not in those without major depression. ${ }^{[215]}$ A controlled trial found that nortriptyline, a noradrenergic antidepressant, reduced drinking in patients diagnosed with antisocial personality disorder, but not those patients with affective/anxiety disorders or those without a comorbid disorder. ${ }^{[216]}$

Finally, there is limited evidence that among schizophrenic patients, two atypical antipsychotics (risperidone and clozapine) may reduce alcohol misuse, smoking, and possibly some other substance misuse. [217]

Currently, only naltrexone and acamprosate are available under the Pharmaceutical Benefits Scheme for alcohol dependence. 
The AUDIT is an appropriate alcohol screening tool for psychiatric populations.

II

Patients with alcohol dependence or abuse are likely to have depression and anxiety symptoms or disorders. Some symptoms may abate once alcohol consumption is reduced or is ceased. However, some patients present with comorbid disorders which require specific intervention (see next point).

Cognitive behavioural therapy appears to be effective in treating clients with comorbid disorders.

Psychosocial interventions above and beyond normal hospital treatment may assist those with schizophrenia.

To date, there is equivocal evidence on the use of SSRI antidepressants as first-line therapy for reducing alcohol consumption in patients with comorbid mood or anxiety disorders. This is a separate issue to their use in treating mood or anxiety disorders.

There is limited evidence that some other pharmacotherapies, for example, desipramine, buspirone, and nortiptyline may reduce alcohol consumption in clients with anxiety or mood disorders. More evidence is required before a strong recommendation can be made.

There is limited evidence that risperidone and clozapine (atypical IV neuroleptics) may reduce alcohol misuse among schizophrenic patients. More evidence is required before a strong recommendation can be made.

\section{RECOMMENDATIONS: COMORBID DISORDERS}

The AUDIT is recommended for screening psychiatric populations. Strong

Assessment for comorbid disorders should take place once the client's withdrawal syndrome has diminished, since some anxiety and depressive symptoms may abate once alcohol consumption is reduced or ceased.

Comorbid mood and anxiety disorders which do not abate after alcohol withdrawal is complete should be treated with integrated/concurrent cognitive behavioural therapy for the cormorbid disorder.

Alcohol dependent patients with comorbid anxiety should be considered for treatment with buspirone, depending upon the sevenity and nature of their symptoms.

Alcohol dependent patients with major depression should be considered for treatment with desipramine, depending upon the severity and nature of their symptoms.

Schizophrenic patients who are risky drinkers should be considered for treatment with clozapine or risperidone, depending upon the severity and nature of their symptoms.

Alcohol dependent patients with antisocial personality disorder should Moderate be considered for treatment with nortiptyline, depending upon the severity and nature of their symptoms. 


\section{Gender}

Much of the research investigating patterns of alcohol use and treatment effectiveness has been conducted in studies focusing either exclusively or primarily on men. ${ }^{[218]}$ However, there is no reason to believe that treatment needs of men and women are the same. There may be different factors associated with risky drinking and alcohol dependence in men and women, and thus treatment may need to adapt according to the gender of the client.

Although risky drinking and alcohol dependence is more common among men than women, a substantial proportion of those who consume alcohol in a risky manner are women. However, most of the research on treatment effectiveness includes only men, and studies that include both men and women often fail to assess gender differences in outcome. Hence, less is known about the characteristics, treatment needs and treatment outcomes of women with alcohol problems than these issues in men.

Studies that have examined the characteristics of women with alcohol and other drug problems suggest that these women may be a distinct group. ${ }^{[218]}$ Because of differences in metabolism, where men and women drink at the same rate, women are at higher risk for liver disease, brain damage, heart disease, violent victimisation, and traffic fatalities. ${ }^{[19]}$ Further, risky alcohol consumption in women who are of childbearing age is particularly problematic as risky alcohol consumption can cause harm to the foetus.

A study of the characteristics of women who receive treatment for alcohol or other drug dependence in Australia reported that more than half of the women were mothers, of whom about one-third did not have custody of their children. ${ }^{[218}$ Polydrug use was the norm, although alcohol use was the primary drug of concern in 20 percent of the sample. Further, a sizeable proportion of the sample in this study suffered from physical and psychological health problems, particularly comorbid anxiety, depression and eating disorders. Almost three-quarters of the sample had experienced physical or sexual violence at some stage in their lives, with 37 percent having been sexually abused in childhood. Thus, women with alcohol problems are likely to present to treatment with a range of problems. Although clinicians cannot be expected to possess the expertise to address all of these issues, the issues should still be identified by clinicians.

In a study of the treatment needs of women, Australian women who had received treatment for alcohol and other drug problems were asked about their experience(s) in treatment, aspects of treatment that they found helpful and aspects perceived as needing improvement. ${ }^{[2201}$ Despite the women's general satisfaction with the services they had received, recommendations about the way in which services address the special needs of women can be made.

First, agencies should try to improve the recruitment of women to their services. Many barriers to seeking treatment were identified. ${ }^{[220]}$ Apart from perceived negative attitudes of general society, and feelings of guilt and shame, many women were unaware of available services and felt that agencies could improve recruitment of women. Possible methods included improving outreach, setting up referral networks, advertising facilities specifically targeting women who may be unaware of available treatment services, and the provision of more detailed information about the services. These methods would increase the awareness of women with problems of treatment services available to them, and increasing knowledge of what treatment involves, may decrease any 
reservations or fears women may have about entering treatment.

The provision of a safe therapeutic environment is essential for both men and women, as the prevalence of physical and sexual abuse is high, particularly among women with alcohol problems. ${ }^{[220]}$ Among the sample in this study, almost three-quarters had experienced physical or sexual violence at some stage in their lives and one-quarter had been sexually or physically harassed while attending a treatment service. Staff should be aware of the potential risks of sexual harassment within residential units and of the need to ensure that clients are protected. Further, women who are admitted to residential or inpatient facilities should have single residential facilities, which would ensure privacy and freedom from sexual harassment.

Treatment agencies should offer both men and women the opportunity to receive information about, and address health and other issues such as depression, anxiety and sexual and physical assault. ${ }^{[220]}$ Staff should be trained to address these issues in a sensitive and appropriate manner, and offer referral to a service, which specialises in the area of concern.

Although it may not always be possible, in treatment agencies where there are clinicians of both gender available to give treatment, patients should be given the choice of clinician gender.

There is a need for treatment providers to be sensitive to issues particular to women with alcohol problems, provide them with a range of treatment options, and refer women to other specialist services where necessary and appropriate. 
Studies that have examined the characteristics of women with alcohol and other drug problems suggest that these women have a range of characteristics which make them distinctive from men.

Because of differences in metabolism, where men and women III-2 drink at the same rate, women are at higher risk for liver disease, brain damage, heart disease, violent victimisation, and traffic fatalities.

Polydrug use and comorbid psychological disorders are common among risky and dependent female drinkers.

There appear to be gender differences in treatment outcome, with women having slightly better results than men in the first twelve months after treatment, and men having slightly better outcomes in follow-ups longer than twelve months.

There is evidence that brief interventions are effective in reducing alcohol consumption in excessive female drinkers.

There is no evidence suggesting which treatment modalities are effective for risky and dependent female drinkers.

Clinicians need to be aware of and sensitive to issues particular to women with alcohol problems.

Treatment agencies should try to improve the recruitment of women to their services by improving outreach, setting up referral networks, advertising facilities specifically targeting women, and providing more detailed information about services. This would increase women's awareness of treatment services available, and may decrease any reservations or fears about entering treatment

All clients should be provided with a safe therapeutic environment, as the prevalence of physical and sexual abuse is high, particularly among women with alcohol problems.

Treatment agencies should offer all clients the opportunity to receive information about, and address health and other issues such as depression, anxiety and sexual and physical assault.

There should be a range of services available to women. Moderate

Women should be referred to other specialist services where necessary and appropriate.

Strong

Where possible, clients should be given the option of having Moderate a clinician of the same or opposite gender, as preferred. 


\section{Indigenous clients}

The harms caused to Australian Indigenous communities by risky alcohol consumption are evident in their higher rates of alcohol-related deaths and greater hospitalisation rates, compared to the non-Indigenous population. This section provides an overview of some of the issues to be considered in providing treatment to Indigenous drinkers. The National Recommendations for the Clinical Management of Alcohol-Related Problems in Indigenous Primary Care Settings provides more detailed advice on the use of direct questioning, overcoming barriers to discussing alcohol within Australia's Indigenous cultures, screening and brief intervention, and treating physical and psychological comorbidities. ${ }^{[5]}$

The key issues from the recommendations include:

- A public health approach is required, that is, interventions at a community and systemic level, to address the social and economic drivers of alcohol misuse. Relapse rates among Indigenous clients who have completed treatment are likely to be high when clients return to a poor socioeconomic situation. Treatment services therefore need to be supported by effective, culturally appropriate interventions at the population level. This should include education about the effects of drinking and the availability of treatment and interventions aimed at improving the socio-economic situation for Indigenous people.

- A person's location affects drug choice and availability, drinking styles and consumption rates. For example, drinkers in a "dry" community are more likely to binge drink irregularly, whereas a person living in a city may have more regular drinking patterns.

- Cultural sensitivity should not be confused with treatment efficacy. Brief interventions may still be effective with Indigenous drinkers, but ways of communicating and knowledge of issues such as gender roles are important issues in their implementation. Hunter, Brady and Hall (2000) ${ }^{[5]}$ state that it would be counterproductive to be prescriptive when the lifestyle and socio-cultural norms of Indigenous Australians are so diverse, but suggest some readings on cross-cultural issues for those who are working with Indigenous clients, including:

$\triangleright \quad$ Brady, M. (1998). The Grog Book: Strengthening Indigenous Community Action on Alcohol. Available from Government information shops in each State or Territory, Cat. No. 9807357.

$\triangleright \quad$ Hollinsworth, D. \& Cunningham, J. (1998). Indigenous Health: Cultural Awareness Program for Medical Education. Produced by Yunggorendi First Nations Centre for Higher Education and Research, Flinders University, South Australia.

$\triangleright \quad$ Eckermann, A. (1992). Binang Goonj. Bridging Cultures in Aboriginal Health. University of New England Press, Armidale.

$\triangleright \quad$ For more readings, see Hunter, Brady \& Hall (2000). ${ }^{[5]}$

- Rates of general practitioner consultations are comparable across Indigenous and non-Indigenous Australians. However, Indigenous people are three times more likely to attend an outpatient clinic than go to a doctor's practice. There is great potential for opportunistic intervention by medical staff in hospitals, and in other settings such as in sobering-up centres, workplaces, comectional facilities, and community health services. The recommendations provide information on and examples of non-judgemental brief interventions. Also see Chapter 7: Brief interventions. Risky and high-nisk drinkers who are not dependent may respond to low-intensity intervention. 
- Although the recommendations suggest the use of the AUDIT for screening alcohol problems, subsequent qualitative research by Brady et al. (2002) ${ }^{[221]}$ suggests that the AUDIT may have limited acceptability with Indigenous health workers: it was thought to be intrusive, question 8 (how often during the last year have you had a feeling of guilt or remorse after drinking?) sometimes needed clarification, and health workers believed that clients sometimes "fudged" their responses. The authors suggest instead using "two carefully worded questions on the frequency of binge drinking, and average daily consumption." p. 378, ${ }^{2211}$. Brief intervention seemed to be culturally appropriate in this particular urban Aboriginal health service. It should be noted that Indigenous clients were not interviewed about their thoughts on the AUDIT. There was also evidence that the health workers became increasingly comfortable with the AUDIT questions over time.

- This same article identified a number of constraints to delivering brief interventions in Indigenous primary-care settings: lack of time, patients who became annoyed when alcohol was raised as an issue in addition to their presenting problem, "...the severity of illness and the complexity of the physical, social and psychological problems with which patients present." p. 378, ${ }^{1221}$. There was some client resistance to intervention, however the authors note that this is probably not specific to Aboriginal settings.

- Experience suggests that clinics in Indigenous settings are often visited by intoxicated individuals. Some intoxicated individuals may present with an insignificant complaint, but may have other concerns they do not volunteer. Communication with intoxicated clients should be direct, in a calm and slow manner, dealing with one thing at a time. It is not the right time to engage in complex health promotion activities.

- Intervention with alcohol dependent clients who present with other problems should deal with the client's primary clinical needs and at the same time draw attention to the contribution of alcohol to the client's problems. Developing and maintaining a non-judgemental and honest relationship is essential to keeping the client engaged in treatment. Dependence indicates abstinence as a goal.

- Medical comorbidity among Indigenous drinkers is common and may include cardiovascular disease, hypertension, respiratory disease, diabetes, liver disease, neurological disonder, for instance, seizures, injury and poisoning, and sexually transmitted infections. Risky alcohol consumption may be associated with violence and sexual assault. Management of comorbid illness may be neglected because of the clinician's reactions to the client's alcohol problem, or by the fatalistic belief that nothing can be done.

- Psychiatric comorbidity, such as anxiety, depression, psychosis is also common. Self-harm and all threats of self-harm should be treated seriously. Primary-care practitioners are advised to develop close working relationships with the relevant area mental health service.

- Not withstanding the high rates of comorbidity among Indigenous clients, and the exclusion of such clients from much of the research, it seems reasonable to assume that research on pharmacotherapies for alcohol dependence, that is, naltrexone, acamprosate and disulfiram) will apply to Indigenous people. However, increasing compliance with the medication may entail dealing with a slightly different set of issues which will, in turn, depend upon the client's cultural environment.

- Practitioners should attempt to remain engaged with drinkers, whether or not the drinking behaviour changes. Many clients do not change their behaviour immediately. In the meantime, support, advice and help can be offered to the client's family and community in 
providing a safe environment while not condoning harmful drinking or associated behaviours. If the client does reduce or stop drinking, they may feel worse for a time. The practitioner's focus should be on helping deal with these, rather than insisting that not drinking always leads to better outcomes.

The services available for Indigenous clients need to provide a greater quality and diversity of treatment options. Particularly in rural areas, one model of treatment (12-step facilitation or AAbased residential treatment) tends to dominate the current services.

\section{RECOMMENDATIONS: INDIGENOUS CLIENTS}

Relapse among Indigenous clients who have completed treatment is likely to be high when clients retum to a poor socioeconomic situation. Treatment services therefore need to be supported by effective, culturally appropriate interventions at the population level.

Clients may present to health care clinics with minor complaints without volunteering information about alcohol consumption. Carefully worded questions about the frequency of heavy drinking and average daily consumption can help to identify risky drinking.

Cultural sensitivity should not be confused with treatment efficacy. For instance brief interventions may still be effective with Indigenous drinkers, but ways of communicating and knowledge of issues such as gender roles are important issues in their implementation.

Increasing compliance with medication such as acamprosate and naltrexone may entail dealing with a slightly different set of issues which will, in tum, depend upon the client's cultural environment.

There is great potential for opportunistic intervention by medical staff in hospitals, and in other settings, such as in sobering-up centres, workplaces, comectional facilities, and community health services.

The services available for Indigenous clients need to provide a greater quality and diversity of treatment options.

Health care practitioners should seek to keep clients engaged in treatment, whether or not the drinking behaviour changes. In the meantime, support can be offered to the drinker's family and community.

Primary care practitioners are advised to develop close working relationships with the relevant area mental health service to help them deal with psychiatric comorbidity.

Communication with intoxicated clients should be direct, in a calm and slow manner, dealing with one thing at a time. It is not the right time to engage in complex health promotion activities.
STRENGTH OF

RECOMMENDATION

Moderate

Strong

Moderate

Moderate

Strong

Strong

Strong

Moderate

Moderate 
Chapter 12: How to put it together 


\section{Chapter 12: How to put it together}

\section{CHAPTER AIM}

This chapter provides a basis for designing an intervention that is appropriate for different levels of dependence and the severity/complexity of problems.

There is a continuum of care from residential to non-residential, and from intensive to brief, and from carefully structured approaches delivered by specialist professional drug and alcohol counsellors through to less structured simple advice from primary health care workers. These components form the overall strategy that now needs to be put in place to deal with alcohol problems in our society. Figures 12.1 and 12.2 below provide an overview of the basic decision making process.

The time available is an important factor in determining the nature and the content of the intervention. Beyond this parameter the severity and complexity of the presenting problem(s) determine duration, setting and content.

The following sections look at situations where there is:

- No time available to intervene with risky drinkers, for instance in emergency wards.

- Brief treatment of risky drinkers, that is, hazardous and early stage problem drinkers identified within a routine screening where the intervention is provided over a few minutes.

- Brief treatment for those drinkers with more severe alcohol problems over a few sessions.

- Longer duration outpatient treatment over a large number of sessions, and over an extended period, where the alcohol problems or dependence suggests the need.

- Inpatient/residential and day patient interventions for the most seriously affected and dependent people.

The emphasis throughout is on providing clinicians with a structured set of procedures that they can use and that are thought likely to maximise the effect of the intervention of choice. 


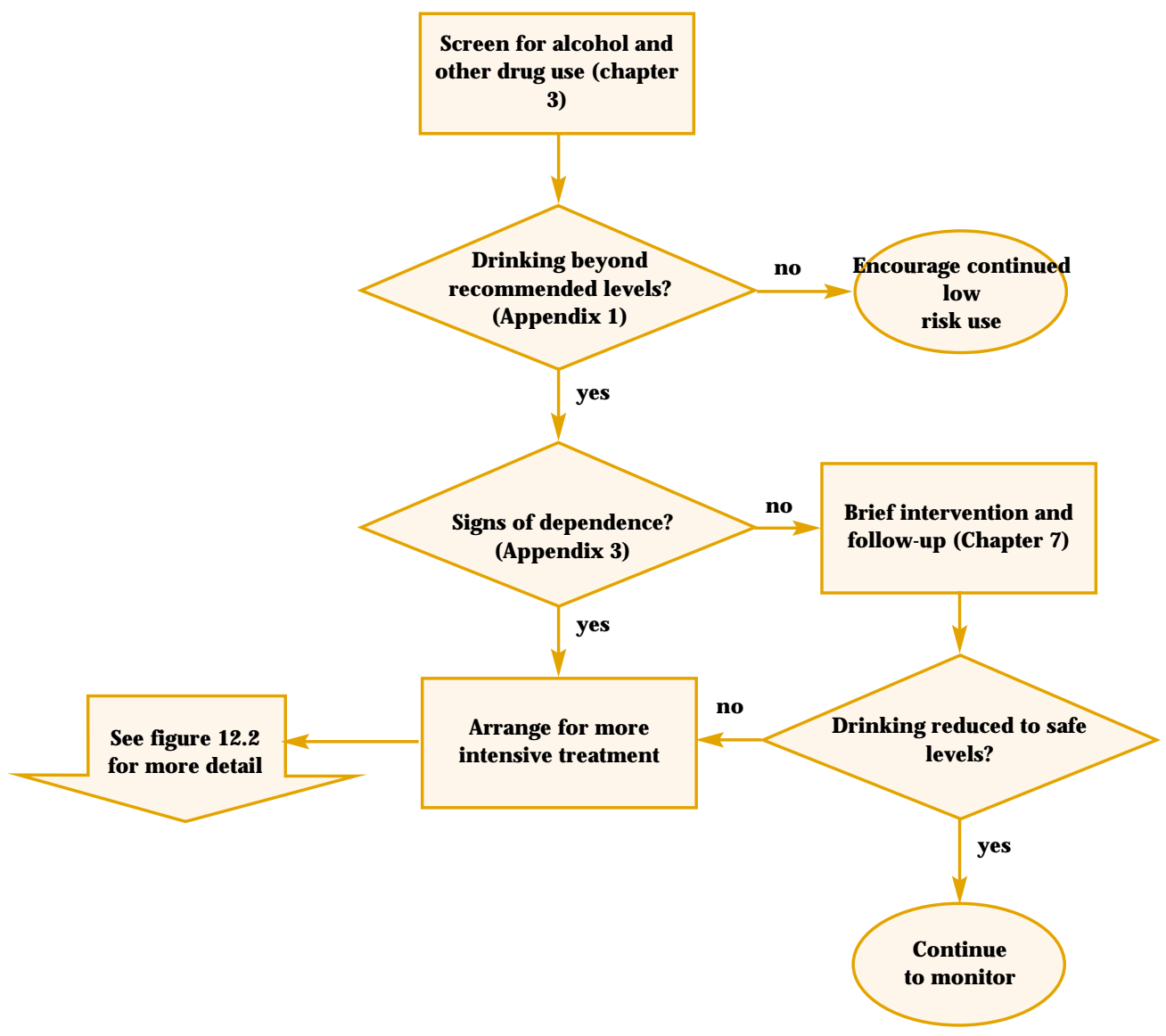




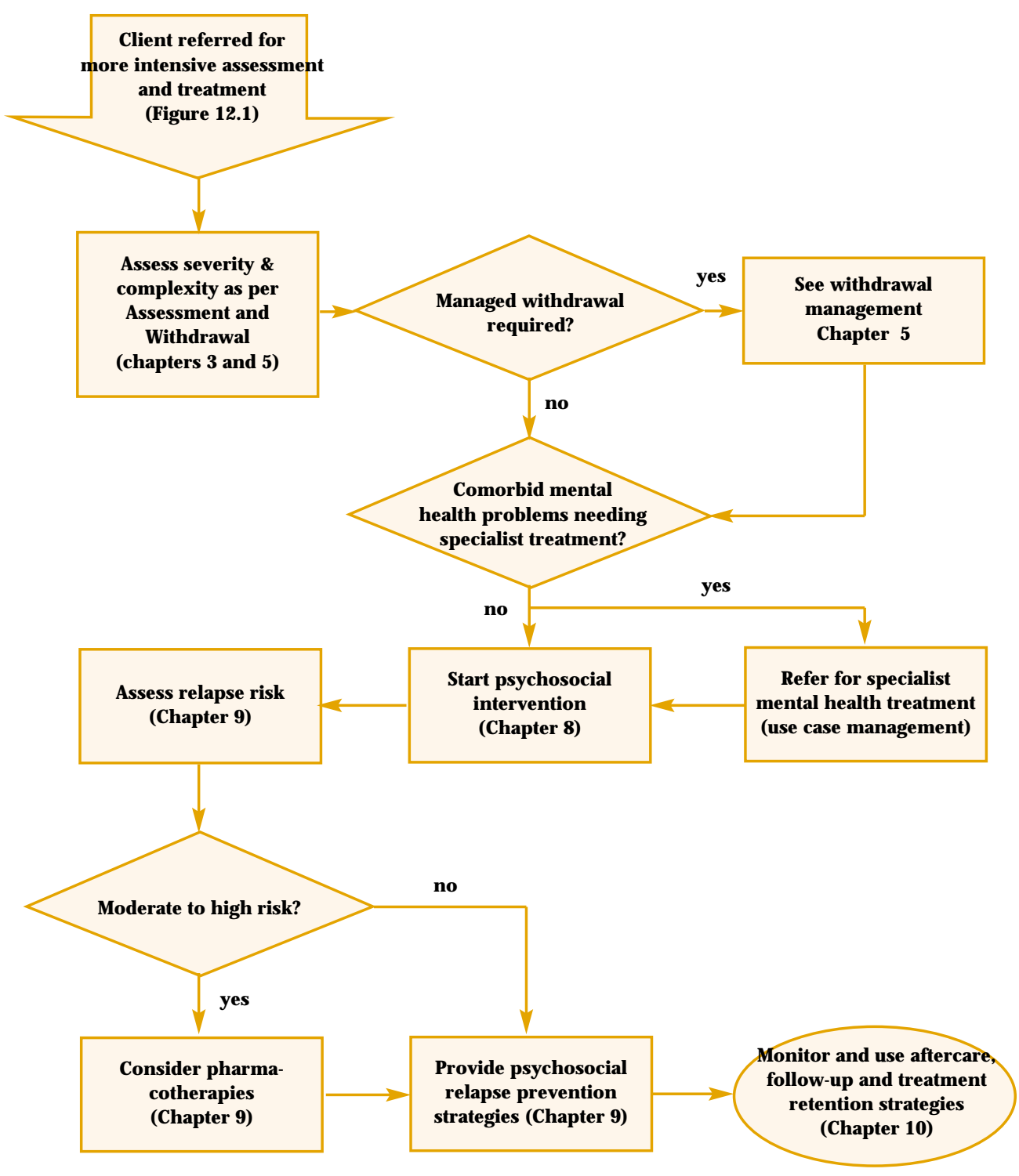




\section{When there is no time available}

In a number of settings there is no time available to screen and detect people with alcohol-related problems, let alone provide an intervention for risky drinking, or alcohol dependence. The most obvious example of such a setting is the accident and emergency wards of larger hospitals. In these settings it is recommended that pamphlets be made available that set out the currently accepted "safe" limits for alcohol consumption, and list the strategies for cutting back drinking, as well as appropriate contact points for referral.

If there is evidence of risky alcohol consumption from the clinical interview there should be a notation to that effect made on the chart or record of the patient to allow later discussion of drinking level, and/or referral for more specialist assistance, as seems necessary. These strategies should be adopted in all general medical, health and welfare settings where it is not possible to deal with the issue of risky drinking directly.

\section{RECOMMENDATIONS}

STRENGTH OF RECOMMENDATION

In those settings that do not allow for screening and Strong intervention with risky drinkers it is recommended that written material setting out the "safe" levels of alcohol consumption, methods for cutting back, and referral points, be made available.

\section{BRIEF, ONE SESSION, FACE-TO-FACE INTERVENTIONS}

Frequently there is time for a brief single session face-to-face intervention, and for many risky drinkers this is all that is required to alter drinking habits. In the context of these brief interventions it is recommended that at a minimum there should be:

- screening and identification of risky alcohol consumption.

- clear and firm advice to cut down consumption.

- description of the safe level of consumption. ${ }^{[34]}$

- a follow-up visit.

It should be possible to deliver that intervention in a relatively brief period. In those settings where there is more time to provide assistance, or where there is a specialist drug and alcohol counsellor providing screening and intervention within a large health care facility:

- The negative health effects of risky consumption should be described in a way that personalises them to the person being counselled, and an empathic counselling style should be used.

- There should be further brief assessment of the extent of the problem and the readiness of the drinker to attempt to reduce consumption.

- Methods of limit setting and general self-management procedures should be discussed.

- If resources allow, a self-help manual/pamphlet should be made available.

- A follow-up visit or some other form of follow-up/referral should be organised.

It is not appropriate in these settings to introduce complex explanations of interventions that require some substantial time and effort in training, such as skills-based approaches. Similarly, the assessment that can be conducted is necessarily brief. 
In brief, one session, face-to-face interventions for Strong risky drinkers, it is recommended that there be clear advice to cut down to a "safe" level of alcohol consumption and that a follow-up visit arranged.

Where there is more time available to counsel the drinker.

- The negative health effects of nisky consumption should be personalised.

- Further brief assessment of the problem and the readiness to reduce consumption conducted and methods of limit setting and self-management should be set out.

A self-help manual made available. Strong

For those whose risky consumption does not decrease, it is recommended that referral for specialist assessment is offered.

\section{BRIEF SESSIONAL OUTPATIENT INTERVENTIONS}

For many clients brief intervention over a few sessions, for example one session up to five sessions will be all that is required, and often all that is wanted by the individual. In this circumstance the treatment should incorporate all of the interventions that are described in Chapter 7: Brief Interventions, and the following:

- An assessment of alcohol dependence and, if there is time, of other areas of the drinker's functioning, especially mental health status (see Chapter 3: Screening and Assessment).

- Motivational interviewing if the drinker appears to be unsure or ambivalent about changing the drinking behaviour (see Chapter 8: Psychosocial interventions).

- The identification of high-risk situations and provision of relapse prevention strategies, including the identification of strategies, such as drink refusal skills to minimise the risk of relapse (see Chapter 9: Relapse prevention).

- Arrangement of a follow-up visit.

Outpatient interventions conducted over a few sessions should include those interventions that have been recommended for brief, one session, face-to-face interventions and: - Assessment of level of dependence and associated problems - A motivational intervention based on the client's readiness for change - Assessment of high-nisk relapse situations and provision of relapse prevention procedures

- A follow-up appointment. 


\section{Longer multi-session outpatient interventions}

Where assessment or previous failures in treatment suggest the need, longer outpatient intervention extended over multiple sessions is required. This form of intervention might occur for up to 15 or more sessions, however no precise guide can (or should) be made about the length of intervention required. In this circumstance the treatment should incorporate all of the interventions that are described in the previous sections, and the following:

- A comprehensive assessment covering motivation to alter drinking patterns, pattern of drinking, dependence on alcohol and need for a supervised detoxification,

psychological/psychiatric problems and disorders, and familial or interpersonal factors that may be exacerbating drinking, and assessment of cognitive functioning if that is necessary (see Chapter 3: Screening and assessment).

- A motivational intervention and personalisation of the health effects of risky drinking, especially if the drinker appears to be unsure or ambivalent about changing the drinking behaviour (see Chapter 8: Psychosocial interventions).

- Referral for intervention for comorbid mental health problems that are detected if there are no suitably skilled staff available in the agency to deal with such problems (see Chapter 11: Interventions for specific client groups).

- Naltrexone or acamprosate should be considered for patients who are medically stable and willing to comply with the medication. Pharmacotherapies should be used only as an adjunct to a comprehensive treatment program.

- After the development of a client-centred relationship aimed to help the client to explore methods of overcoming their dependence, the use of selected training in skills-based approaches as necessary to deal with communication skills deficits, social skills deficits, lack of assertiveness, relaxation or stress management problems, and relationship difficulties may be helpful (see Chapter 8: Psychosocial interventions).

- An introduction to Alcoholics Anonymous (AA) may be discussed (see Chapter 10: Extended care).

- Relapse prevention should be undertaken to minimise the risk of a return to problem drinking (see Chapter 9: Relapse prevention).

- Scheduled aftercare or booster sessions should form an integral part of the intervention.

\begin{tabular}{l|c} 
RECOMMENDATIONS & $\begin{array}{c}\text { STRENGTH OF } \\
\text { RECOMMENDATION }\end{array}$
\end{tabular}

It is recommended that in the case of extended outpatient Strong intervention that there be:
a comprehensive assessment
referral for treatment of serious comorbid disorders
introduction of pharmacotherapy if appropriate
the development of a client-centred relationship and
training in selected skills based approaches
relapse prevention
referral to AA when there is a goal of abstinence

Aftercare, via scheduled booster or follow-up sessions, should

Strong

be an integral part of the treatment package. 


\section{Brief inpatient/residential withdrawal management}

The guidelines for managing withdrawal from alcohol are set out in Chapter 5. Many brief inpatient programs aimed at detoxification can provide some intervention for the drinker. Patient's needs for further intervention should be assessed after detoxification. A motivational interview incorporating the personalisation of health effects should be conducted and the range of treatment options available should be fully described to the drinker. Where the goal of abstinence seems appropriate the drinker may be linked into an AA group that is suitable to his or her background and characteristics. Thereafter the patient should be offered an outpatient or residential intervention as required, based on the assessment.

\begin{tabular}{l|c}
\hline RECOMMENDATIONS & $\begin{array}{l}\text { STRENGTH OF } \\
\text { RECOMMENDATION }\end{array}$ \\
\hline $\begin{array}{l}\text { It is recommended that withdrawal facilities should assess } \\
\text { drinkers for further intervention, provide a motivational } \\
\text { intervention where necessary, and describe the range of } \\
\text { interventions available. }\end{array}$ & $\begin{array}{l}\text { Strong } \\
\end{array}$ \\
\hline
\end{tabular}

\section{Day patient and inpatient/residential interventions}

For some clients, assessment indicates that a more intensive and extensive day patient or residential intervention conducted over several weeks is required. This is the case where outpatient treatment has repeatedly failed and where the severity of the problems indicates the need. The circumstances wherein such an intervention is indicated are set out in Chapter 6 . Where inpatient/residential intervention is desirable, the treatment should incorporate all of the interventions that are described in the previous sections, and the following:

- A comprehensive assessment covering motivation to alter drinking patterns, pattern of drinking, dependence on alcohol and need for a supervised detoxification, psychological/psychiatric problems and disorders, and familial or interpersonal factors that may be exacerbating drinking, and an assessment of cognitive functioning if that seems necessary (see Chapter 3: Post-withdrawal treatment setting).

- A motivational intervention and personalisation of the health effects of risky drinking, especially if the drinker appears to be unsure or ambivalent about changing the drinking behaviour (see Chapter 8: Psychosocial interventions).

- Referral for intervention for comorbid mental health problems that are detected if there are no suitably skilled staff available in the agency to deal with such problems (see Chapter 11: Interventions for specific client groups).

- Naltrexone or acamprosate should be considered for patients who are medically stable and willing to comply with the medication. Pharmacotherapies should be used only as an adjunct to a comprehensive treatment program (see Chapter 9: Relapse prevention).

- After the development of a client-centred relationship aimed to help the client to explore methods of overcoming their dependence, the use of selected training in skills-based approaches as necessary to deal with communication skills deficits, social skills deficits, lack of assertiveness, relaxation or stress management problems, and relationship difficulties may be helpful (see Chapter 8: Psychosocial interventions). 
- An introduction to AA may be arranged (see Chapter 10: Extended care).

- Relapse prevention intervention should be undertaken to minimise the risk of a return to problem drinking (see Chapter 9: Relapse prevention).

- A reintegration or "re-entry" phase that assists the drinker to make a transition from institutional care to self-care in the community should be included.

- Scheduled aftercare or booster sessions should form an integral part of the intervention, and should include professionally delivered relapse prevention procedures plus self-help if appropriate.

\section{RECOMMENDATIONS}

It is recommended that intensive day patient or residential intervention proceed along similar lines to outpatient treatment. It should involve:

- comprehensive assessment

- referral for serious comorbid disorders

- introduction of pharmacotherapies if appropriate

- training in selected skills

- relapse prevention

- referral to AA when the goal is abstinence

There should be a re-entry phase to the intervention wherein the client is assisted in returning to his or her usual environment while not relapsing to drinking.

\section{STRENGTH OF RECOMMENDATION}

\section{Strong}




\section{References}




\section{Refierences}

1. Mattick, R.\& Jarvis, T. (1993). An outline for the management of alcohol problems: Quality Assurance in the Treatment of Drug Dependence Project. Monograph No. 20. National Drug Strategy, Commonwealth Department of Human Services and Health: Canberra.

2. National Health and Medical Research Council. (2000). How to use the evidence: assessment and application of scientific evidence. National Health and Medical Research Council: Canberra.

3. Andrews, G. (1984). On the promotion of non-drug treatments. British Medical Journal, 289, 994-995.

4. Joint Advisory Group on General Practice and Population Health (2001). Smoking, Nutrition, Alcohol and Physical Activity (SNAP) Framework for General Practice. Commonwealth Department of Health and Aged Care: Canberra.

5. Hunter, E., Brady, M., \& Hall, W. (2000). National Recommendations for the Clinical Management of Alcohol-related Problems in Indigenous Primary Care Settings, Commonwealth Department of Health and Aged Care: Canberra.

6. Australian Institute of Health \& Welfare. (2002). 2001 National Drug Strategy Household Survey: First results. AIHW, Canberra. www.aihw.gov.au:

7. National Institute of Alcohol Abuse and Alcoholism. (1998). Alcohol and aging, in Alcohol Alert No. 40. NIAAA: Bethesda, MD.

8. Holder, H., Longabaugh, R, Miller, W., \& Rubonis, A. (1991). The cost effectiveness of treatment for alcoholism: a first approximation. Journal of Studies on Alcohol, 52(6), 517-540.

9. Wutzke, S., Shiell, A., Gomel, M., \& Conigrave, K. (2001). Cost effectiveness of brief interventions for reducing alcohol consumption. Social Science and Medicine, 52, 863-870.

10. Babor, T. \& Higgins-Biddle, J. (2000). Alcohol screening and brief intervention: dissemination strategies for medical practice and public health. Addiction, 95(5), 677-686.

11. Roche, A., Guray, C., \& Saunders, J. (1991). General practitioners' experiences of patients with drug and alcohol problems. British Journal of Addiction, 86, 263-275.

12. Richmond, R, Kehoe, L, Heather, N., \& Wodak, A. (2000). Evaluation of a workplace brief intervention for excessive alcohol consumption: the workscreen project. Preventative Medicine, 30(1), 51-63.

13. Babor, T.\& Higgins-Biddle, J. (2000). Alcohol screening and brief intervention: dissemination strategies for medical practice and public health. Addiction, 95(5), 677-686. 
14. Dawe, S., Loxton, N., Hides, L, Kavanagh, D., \& Mattick, R. (2002). Review of diagnostic screening instruments for alcohol and other drug use and other psychiatric disorders (2nd edition). Commonwealth Department of Health and Ageing: Canberra.

15. Saunders, J., Aasland, O., Babor, T., de la Fuente, J., \& Grant, M. (1993). Development of the Alcohol Use Disorders Identification Test (AUDIT). WHO collaborative project on early detection of persons with harmful alcohol consumption - II. Addiction, 88, 791804 .

16. Babor, T., de la Fuente, J., Saunders, J., \& Grant, M. (1992). The Alcohol Use Disorders Identification Test: Guidelines for use in Primary Health Care. World Health Organization.

17. Piccinelli, M., Tessari, E., Bortolomasi, M., Piasere, O., Semenzin, M., Garzotto, N., \& Tansella, M. (1997). Efficacy of the Alcohol Use Disorders Identification Test as a screening tool for hazardous alcohol intake and related disorders in primary care: a validity study. British Medical Journal, 314, 420-424.

18. Bush, K., Kivlahan, D., McDonell, M., Fihn, S., \& Bradley, K. (1998). The AUDIT alcohol consumption questions (AUDIT-C): an effective brief screening test for problem drinking. Archives of International Medicine, 158, 1789-1794.

19. Selzer, M. (1971). The Michigan Alcoholism Screening Test: The quest for a new diagnostic instrument. American Journal of Psychiatry, 127, 85-94.

20. Mayfield, D., McLeod, G., \& Hall, P. (1974). The CAGE questionnaire: Validation of a new alcoholism screening instrument. American Journal of Psychiatry, 131, 1121-1123.

21. Drummond, D. (1990). The relationship between alcohol dependence and alcohol-related problems in a clinical population. British Journal of Addiction, 85(3), 357-366.

22. Williams, B., \& Drummond, D. (1994). The Alcohol Problems Questionnaire: reliability and validity. Drug \& Alcohol Dependence, 35(3), 239-244.

23. Ryder, D., Lenton, S., Harrison, S., \& Dorricott, J. (1988). Alcohol-related problems in a general hospital and a general practice: screening and the preventive paradox. Medical Journal of Australia, 149, 355-360.

24. Rydon, P. (1991). Detection of alcohol problems in general practice: development of the Newcastle alcohol-related problems scale. University of Newcastle: Newcastle.

25. Waterson, E.\& Murray-Lyon, I. (1988). Are the CAGE questions outdated? British Journal of Addiction, 83, 1113 - 1115.

26. Russell, M., Martier, S., Sokol, R., \& Mudar, P. (1994). Screening for pregnancy risk-drinking. Alcoholism, Clinical and Experimental Research, 18, 1156-1161. 
27. World Health Organization. (1992). The Alcohol Use Disorders Identification Test: Guidelines for use in primary health care. World Health Organization.

28. Saunders, J.\& Conigrave, K. (1990). Early identification of alcohol problems. Canadian Medical Association Journal, 143, 1060-1068.

29. Skinner, H., Holt, S., Sheu, W., \& Israel, Y. (1986). Clinical versus laboratory detection of alcohol abuse: the Alcohol Clinical Index. British Medical Journal, 292, 1703-1708.

30. Yang, M., \& Skinner, H. (2002). Assessment for brief intervention and treatment, in International Handbook of Alcohol Dependence and Problems. N. Heather, T.J.Peters, T. Stockwell, Editor. John Wiley \& Sons: Chichester.

31. Vanclay, F., Raphael, B., Dunne, M., Whitfield, J., Lewin, T., \& Singh, B. (1991). A community screening test for high alcohol consumption using biochemical and haematological measures. Alcohol \& Alcoholism, 26, 337-346.

32. Musshoff, F.\& Daldrup, T. (1998). Determination of biological markers for alcohol use. Journal of Chromatography B, 713(1), 245-264.

33. Shakeshaft, A., Bowman, J., \& Sanson-Fisher, R. (1998). Comparison of three methods to assess binge consumption: one-week retrospective drinking diary, AUDIT, and quantity/frequency. Substance Abuse, 19(4), 191-203.

34. National Health \& Medical Research Council. (2001). Australian Alcohol Guidelines: Health Risks and Benefits. NHMRC: Canberra.

35. Shakeshaft, A., Bowman, J., \& Sanson-Fisher, R. (1999). A comparison of two retrospective measures of weekly alcohol consumption: diary and quantity/frequency index. Alcohol \& Alcoholism, 34(4), 636-645.

36. Miller, W. (1995). Increasing Motivation for Change, in Handbook of Alcoholism Treatment Approaches: Effective Alternatives, R.K. Hester, W.R. Miller, Editors. Allyn \& Bacon: Boston.

37. Ritter, A., Bowden, S., Murray, T., Ross, P., Greeley, J., \& Pead, J. (2002). The influence of the therapeutic relationship in treatment for alcohol dependency. Drug \& Alcohol Review, 21261-268.

38. Fiorentine, R., Nakashima, J., \& Anglin, D. (1999). Client engagement in drug treatment. Journal of Substance Abuse Treatment, 17(3), 199-206.

39. Joe, G., Simpson, D., Greener, J., \& Rowan-Szal, G. (1999). Integrative modeling of client engagement and outcomes during the first 6 months of methadone treatment. Addictive Behaviors, 24(5), 649-659.

40. Miller, W. (1987). Motivation and treatment goals. Drugs and Society, 11, 33-151.

41. Sanchez-Craig, M. (1990). Brief didactic treatment for alcohol and drug-related problems: an approach based on client choice. British Journal of Addiction, 85(2), 169-177.

42. Rokke, P., Tomhave, J., \& Jocic, Z. (1999). The role of client choice and target selection 
in self-management therapy for depression in older adults. Psychology \& Aging, 14(1), 155-169.

43. Brown, J.\& Miller, W. (1993). Impact of motivational interviewing on participation and outcome in residential and alcoholism treatment. Psychology of Addictive Behavior, 7(4), 211-218.

44. Richmond, R., Webster, I., Elkins, L., Mendelson, C., \& Rollnick, S. (1991). Smokescreen for the 1990s: The stop smoking programme for use by general practitioners. University of New South Wales: Sydney.

45. Prochaska, J., \& DiClemente, C. (1986). Toward a comprehensive model of change., in Treating addictive behaviors: Processes of change, W.R. Miller, N. Heather, Editors. Plenum Press: New York. p. 3-27.

46. Belding, M., Iguchi, M., \& Lamb, R. (1997). Stages and processes of change as predictors of drug use among methadone maintenance patients. Experimental \& Clinical Psychopharmacology, 5(1), 65-73.

47. Edens, J. \& Willoughby, F. (2000). Motivational patterns of alcohol dependent patients: a replication. Psychology of Addictive Behaviors, 14(4), 397-400.

48. Willoughby, F.W. \& Edens, J.F. (1996). Construct validity and predictive utility of the stages of change scale for alcoholics. Journal of Substance Abuse., 8(3), 275-91.

49. Davidson, R. (1998). The Transtheoretical Model: A Critical Overview, in Treating Addictive Behaviors, W.R. Miller, N. Heather, Editors. Plenum Press: New York.

50. Littell, J.H.\& Girvin, H. (2002). Stages of change. A critique. Behavior Modification, 26(2), 223-73.

51. Whitelaw, S., Baldwin, S., Bunton, R., \& Flynn, D. (2000). The status of evidence and outcomes in Stages of Change research. Health Education Research, 15(6), 707-18.

52. Rollnick, S. (1998). Readiness, Importance, and Confidence: Critical Conditions of Change in Treatment, in Treating Addictive Behaviors, W.R. Miller, N. Heather, Editors. Plenum Press: New York.

53. Carney, M. \& Kivlahan, D. (1995). Motivational subtypes among veterans seeking substance abuse treatment: Profiles based on stages of change. Psychology of Addictive Behaviors, 9(2), 135-142.

54. Heather, N., Rollnick, S., \& Bell, A. (1993). Predictive validity of the Readiness to Change Questionnaire. Addiction, 88(12), 1667-77.

55. Gavin, D.R., Sobell, L.C., \& Sobell, M.B. (1998). Evaluation of the readiness to change questionnaire with problem drinkers in treatment. Journal of Substance Abuse, 10(1), 53-8.

56. Edwards, G. (1982). The treatment of drinking problems: A guide for the helping professions. London: Grant MacIntyre.

57. Sobell, L \& Sobell, M. (1992). Timeline follow-back: A technique for assessing self- 
reported alcohol consumption, in Measuring Alcohol Consumption, R. Litten, J.Allen, Editors. Humana Press Inc.

58. Miller, W. (1984). Brief Drinker Profile. Odessa, Florida: Psychological Assessment Disorders.

59. Miller, W. \& Marlatt, G. (1984). Comprehensive Drinker Profile. Odessa, Florida: Psychological Assessment Resources.

60. American Psychiatric Association. (1994). Diagnostic and Statistical Manual of Mental Disorders, 4th Edition. Washington: American Psychiatric Association.

61. Stockwell, T., Sitharthan, T., McGrath, D., \& Lang, E. (1994). The measurement of alcohol dependence and impaired control in community samples. Addiction, 89, 167-174.

62. Raistrick, D., Dunbar, G., \& Davidson, R. (1983). Development of a questionnaire to measure alcohol dependence. British Journal of Addiction, 78, 89-95.

63. Meehan, J., Webb, M., \& Unwin, A. (1985). The Severity of Alcohol Dependence Questionnaire (SADQ) in sample of Irish problem drinkers. British Journal of Addiction, 80(1), 57-63.

64. Skinner, H. \& Horn, J. (1984). Alcohol Dependence Scale (ADS): User's Guide. Toronto: Addiction Research Foundation.

65. Heather, N. (1989). Brief intervention strategies, in Handbook of alcoholism treatment approaches, R. Hester, W.R. Miller, Editors. Pergamon Press: New York. p. 93-116.

66. Degenhardt, L (2000b). Comorbidity between substance use and mental health in Australia: Relationships of alcohol, tobacco and cannabis use with other substance use and mental disorders, in Thesis, School of Psychology and National Drug and Alcohol Research Centre. University of New South Wales: Sydney, Australia. p. 443.

67. Beck, A. \& Steer, R. (1987). Beck depression inventory: Manual. USA: Harcourt, Brace, Jovanovich.

68. Beck, A.\& Steer, R. (1990). Beck anxiety inventory: Manual. USA: Harcourt, Brace, Jovanovich.

69. Derogatis, L. (1983). SCL-90-R: Administration, scoring and procedures manual - II for the revised version. Towson, MD, USA: Clinical Psychometric Research.

70. Lovibond, S. \& Lovibond, P. (1993). Manual for the Depression Anxiety Stress Scales. Sydney: Psychology Foundation, University of New South Wales: Sydney.

71. Copeland, J. \& Hall, W. (1992). A comparison of women seeking drug and alcohol treatment in a specialist women's and two traditional mixed-sex treatment services. British Journal of Addiction, 87, 1293-1302. 
72. Goldberg, D. (1972). The detection of psychiatric illness by questionnaire. London, UK: Oxford University Press.

73. Ware, J., Jr., Kosinski, M., \& Keller, S.D. (1996). A 12-Item Short-Form Health Survey: construction of scales and preliminary tests of reliability and validity. Medical Care, 34(3), 220-33.

74. Beck, A.\& Steer, R. (1987). Beck Hopelessness Scale Manual. San Antonio: The Psychological Corporation. Harcourt Brace Jovanovich Inc.

75. Speilberger, C., Gorsuch, R., Lushene, R., Vagg, P., \& Jacobs, G. (1983). Manual for the State-Trait Anxiety Inventory (Form Y). Palo Alto: Consulting Pyschologist Press, Inc.

76. Mattick, R.P.\& Clarke, J.C. (1998). Development and validation of measures of social phobia scrutiny fear and social interaction anxiety. Behaviour Research \& Therapy, 36(4), 455-70.

77. Falsetti, S., Resnick, H., Resick, P., \& Kilpatrick, D. (1993). The Modified PTSD Symptom Scale: A brief self-report measure of posttraumatic stress disorder. The Behavioral Therapist, 16, 161-162.

78. Bryant, R., Moulds, M., \& Guthrie, R. (2000). Acute stress disorder scale: A self-report measure of acute stress disorder. Psychological Assessment, 12(1), 61-68.

79. Garner, D.M.\& Garfinkel, P.E. (1979). The Eating Attitudes Test: an index of the symptoms of anorexia nervosa. Psychological Medicine, 9(2), 273-9.

80. Kessler, R. (2002). Short screening scales to monitor population prevalences and trends in nonspecific psychological distress. Psychological Medicine, 32(6), 959-976.

81. Cook, C. (2000). Prevention and treatment of Wernicke-Korsakoff syndrome. Alcohol and Alcoholism, 35(Suppl. 1), 19-20.

82. Lishman, W. (1987). Organic psychiatry: psychological consequences of cerebral disorder (2nd ed.). Oxford: Blackwell Scientific Publications.

83. Goldman MS. (1995). Recovery of Cognitive Functioning in Alcoholics: The Relationship to Treatment. Alcohol Health and Research World, 19(2), 148-154.

84. Folstein, M., Folstein, S., \& McHugh, P. (1975). Mini-Mental State: A practical method for grading the cognitive status of patients for the clinician. Journal of Psychiatric Research, 12, 189-198.

85. Alderman, C. \& Peter, P. (1995). Mini-Mental State Examination alone may not adequately rule out the possibility of cognitive deficit (letter, comment). Medical Journal of Australia, 163(1), 53.

86. Ryan, J., Arb, J., Paul, C., \& Kreiner, D. (2000). Reliability of the WAIS-III subtests, indexes, and IQs in individuals with substance abuse disorders. Assessment, 7(2), 151156.

87. Lennane, K. (1986). Management of moderate to severe alcohol-related brain damage 
(Korsakoff's syndrome). Medical Journal of Australia, 145, 136-143.

88. Miller, W., Sovereign, R, \& Krege, B. (1988). Motivational interviewing with problem drinkers; II. The Drinker's Check-up as a preventative intervention. Behavioural Psychotherapy, 16, 251-268.

89. Mattson, M.E. (1994). Patient-treatment matching. Alcohol Health and Research World, 18(4), 287-295.

90. Project MATCH Research Group. (1997). Matching alcoholism treatments to client heterogeneity: Project MATCH posttreatment drinking outcomes. Journal of Studies in Alcohol, 58(1), 7-29.

91. Yost, D. (1996). Alcohol Withdrawal Syndrome. American Family Physician, 54(2), 657659.

92. Foy, A., Kay, J., \& Taylor, A. (1997). The course of alcohol withdrawal in a general hospital. Quarterly Journal of Medicine, 90(253-271).

93. Sellers, E. \& Romach, M. (1991). Pharmacotherapy of alcohol and drug problems. Drug and Alcohol Review, 10(3), 215-224.

94. Frank, L \& Pead, J. (1995). New concepts in drug withdrawal: A resource handbook. Department of Public Health and Community Medicine, Victoria.

95. MIMS Australia. (2002). MMS Annual 2002. 26th Edition ed. Singapore: Tien Wah Press Pty Ltd.

96. D’Onofrio, G., Rathlev, N., Ulrich, A., Fish, S., \& Freedland, E. (1999). Lorazepam for the prevention of recurrent seizures related to alcohol. The New England Journal of Medicine, 340(12), 915-919.

97. NSW Health Department. (1999). NSW Detoxification Clinical Practice Guidelines. Sydney: Better Health Centre.

98. Saitz, R\& O'Malley, S. (1997). Pharmotherapies for alcohol abuse: Withdrawal and Treatment. Alcohol and Other Substance Abuse, 81(4), 881-906.

99. Sullivan, J., Sykora, K., Schneiderman, J., Naranjo, C., \& Sellers, E. (1989). Assessment of Alcohol Withdrawal: the revised clinical institute withdrawal assessment for alcohol scale (CIWA-Ar). British Journal of Addiction, 84, 1353-1357.

100. Novak, H., Blayney, R., Hanagan, M., Moor, S., Symes, A., \& Saunders, J. (1989). Guidelines for nursing management of patients who are intoxicated or withdrawing from alcohol, Directorate of the Drug Offensive, NSW Health: Rozelle, NSW.

101. Gossop, M., Keaney, F., Stewart, D., Marshall, E., \& Strang, J. (2002). A short Alcohol Withdrawal Scale (SAWS): development and psychometric properties. Addiction Biology, 7, 37-43.

102. Saunders, J., Ward, H., \& Novak, H. (1996). Guide to Home Detoxification. Sydney: Drug and Alcohol Department, Central Sydney Area Health Service, National Drug Strategy.

103. Better Health Good Health Care. (2000). Alcohol and Other Drugs Policy for Nursing 
Practice in NSW: Clinical Guidelines. NSW Department of Health: Sydney.

104. Hillborn, M., Tokola, R., Kuusela, V., Karkkainen, P., Kalli-Lemma, L, Pilke, A., \& Kaste, M. (1989). Prevention of alcohol withdrawal seizures with carbamazepine and valproic acid. Alcohol, 6, 223-226.

105. Swift, R. (1997). Pharmacological Treatment of Alcoholism: Clinical Management, in The principles and practice of addictions in psychiatry, S Miller (Ed.), Editor. W. B. Saunders Company: Philadelphia. p. 467-478.

106. Gill, T. (1997). GP Drug and Alcohol Supplement, in Detoxification from alcohol. Central Coast Area Health Service, New South Wales, Australia.

107. Rychtarik, R., Connors, G., Whitney, R., McGillicuddy, N., Fitterling, J., \& Wirtz, P. (2000). Treatment settings for persons with alcoholism: evidence for matching clients to inpatient versus outpatient care. Journal of Consulting \& Clinical Psychology, 68(2), 277-89.

108. Keso, L \& Salaspuro, M. (1990). Inpatient treatment of employed alcoholics: A randomized clinical trial on Hazelden-type and traditional treatment. Alcoholism: Clinical \& Experimental Research, 14, 584-589.

109. De Leon, G., Sacks, S., Staines, G., \& McKendrick, K. (2000). Modified therapeutic community for homeless mentally ill chemical abusers: treatment outcomes. American Journal of Drug \& Alcohol Abuse, 26(3), 461-480.

110. Gowing, L, Cooke, R., Biven, A., \& Watts, D. (in press). Towards Better Practice in Therapeutic Communities. Australasian Therapeutic Communities Association.

111. Melnick, G., De Leon, G., Thomas, G., \& Kressel, D. (2001). A client-treatment matching protocol for therapeutic communities: first report. Journal of Substance Abuse Treatment, 21, 119-128.

112. Yohman, J., Schaeffer, K., \& Parson, O. (1988). Cognitive training in alcoholic men. Journal of Consulting \& Clinical Psychology, 56, 67-72.

113. Morgenstern, J. \& Bates, M. (1999). Effects of executive function impairment on change processes and substance use outcomes in 12-step treatment. Journal of Studies on Alcohol, 60(6), 846-855.

114. De Leon, G., Hawke, J., Jainchill, N., \& Melnick, G. (2000). Therapeutic communities. Enhancing retention in treatment using "Senior Professor" staff. Journal of Substance Abuse Treatment, 19(4), 375-382.

115. Moyer, A., Finney, J., Swearington, C., \& Vergun, P. (2002). Brief interventions for alcohol problems: a meta-analytic review of controlled investigations in treatment -seeking and non-treatment seeking populations. Addiction, 97, 279-292.

116. Bien, T., Miller, W., \& Tonigan, J. (1993a). Brief interventions for alcohol problems: a review. Addiction, 88(3), 315-355. 
117. Heather, N. (2001). Brief Interventions, in International Handbook of Alcohol Dependence and Problems, N. Heather, T.J. Peters, T. Stockwell, Editors. John Wiley \& Sons, Ltd: New York.

118. Doran, C., Fawcett, J., Shakeshaft, A., Shanahan, M., \& Mattick, R. (in preparation). General Practitioners' role in preventive medicine: Scenario analysis using alcohol as a case study.

119. Britt, H., Miller, G., Knox, S., Charles, J., Valenti, L, Henderson, J., Kelly, Z., \& Pan, Y. (2001). General Practice Activity in Australia 2000-01. Australian Institute of Health and Welfare (General Practice Series No. 8): Canberra.

120. Bell, A., Richmond, R., Heather, N., \& Rollnick, S. (1994). Results of screening for excessive drinkers in four Sydney teaching hospitals. Drug and Alcohol Review, 13, 179184.

121. Heather, N., Rollnick, S., Bell, A., \& Richmond, R. (1996). Effects of brief counselling among male heavy drinkers identified on general hospital wards. Drug and Alcohol Review, 15(1), 29-38.

122. Gentilello, L, Rivara, F., Donovan, D., Jurkovich, G., Daranciang, E., Cunn, C., Villaveces, A., Copass, M., \& Ries, R. (1999). Alcohol interventions in a trauma center as a means of reducing the risk of injury recurrence. Annals of Surgery, 230(4), 473-483.

123. Galarza, N., Diaz, R., Guzman, F., Caballero, J., \& Martinez, A. (1997). The use of naltrexone to treat ambulatory patients with alcohol dependence. Boletin - Asociacion Medica de Puerto Rico, 89(10-12), 157-160.

124. Gentilello, L, Duggan, P., Drummond, D., Tonnesen, A., Degner, E., Fischer, R., Reed, R. \& . (1988). Major injury as a unique opportunity to initiate treatment in the alcoholic. American Journal of Surgery, 156, 558-561.

125. Longabaugh, R., Minugh, P., Nirenberg, T., Clifford, P., Becker, B., \& Woolard, R. (1995). Injury as a motivator to reduce drinking. Academic Emergency Medicine, 2, 817-825.

126. Minugh, P., Nirenberg, T., Clifford, P., Longabaugh, R., Becker, B., \& Wooland, R. (1997). Analysis of alcohol use clusters among subcritically injured emergency department patients. Academic Emergency Medicine, 4, 1059-1067.

127. Richmond, R. Wodak, A., Bourne, S., \& Heather, N. (1998). Screening for unhealthy lifestyle factors in the workplace. Australia and New Zealand Journal of Public Health, 22, 324-331.

128. Richmond, R., Wodak, A., Kehoe, L, \& Heather, N. (1998). How healthy are the police? A survey of lifestyle factors. Addiction, 93(11), 1729-1737.

129. Richmond, R., Kehoe, L, Hailstone, S., Wodak, A., \& Uebel-Yan, M. (1999). Quantitative and qualitative evaluations of brief interventions to change excessive drinking, smoking and stress in the police force. Addiction, 94(10), 1509-1521.

130. Carroll, K. (2001). Constrained, confounded and confused: why we really know so little about therapists in treatment outcome research. Addiction, 96, 203-206. 
131. Finney, J. \& Moos, R. (2002). Psychosocial treatments for alcohol use disorders, in A guide to treatments that work, P. Nathan, Gorman JM., Editor. Oxford University Press: London, England. p. 157-168.

132. Najavits, L. \& Weiss, R. (1994). Variations in therapist effectiveness in the treatment of patients with substance use disorders: an empirical review. Addiction, 89, 679-688.

133. Najavits, L, Crits-Christoph, P., \& Dierberger, A. (2000). Clinicians' impact on the quality of substance abuse disonder treatment. Substance Use and Misuse, 35(12-14), 2161-2190.

134. Miller, W., Benefield, R., \& Tonigan, J. (1993). Enhancing motivation for change in problem drinking: A controlled comparison of two therapist styles. Journal of Consulting and Clinical Psychology, 61, 455-461.

135. Broome, K., Simpson, D., \& Joe, G. (1999). Patient and program attributes related to treatment process indicators in DATOS. Drug \& Alcohol Dependence, 57(2), 127-135.

136. Crits-Christoph, P. (1991). Meta-analysis of therapist effects in psychotherapy outcome studies. Psychotherapy Research, 1(2), 81-91.

137. Egan, G. (2002). The skilled helper: A problem-management and opportunity-development approach to helping. $7^{\text {th }}$ ed. California: Brooks-Cole.

138. Miller, W.R. \& Rollnick, S. (1991). Motivational interviewing: preparing people to change addictive behavior. New York: The Guilford Press.

139. Miller, W.R. \& Rollnick, S. (2002). Motivational Interviewing: Preparing people for change. $2^{\text {nd }}$ ed. New York: The Guilford Press.

140. Miller, W. \& Wilbourne, P. (2002). Mesa Grande: a methodological analysis of clinical trials of treatments for alcohol use disorders. Addiction, 97, 265-277.

141. Marlatt, G. \& Gordon, J. (1985). Relapse Prevention: Maintenance Strategies in the Treatment of Addictive Behaviors. New York: The Guildford Press.

142. Jarvis, T., Tebbutt, J., \& Mattick, R. (1995). Treatment approaches for alcohol and drug dependence. West Sussex, England: John Wiley and Sons.

143. Connors, G., Tonigan, J., \& Miller, W. (2001). A longitudinal model of intake symptomatology, AA participation and outcome: retrospective study of the Project MATCH outpatient and aftercare samples. Journal of Studies on Alcohol, 62(6), 817-825.

144. Beamish, P.M.G., Darcy Haag; Belcastro, Amy L (2002). Treatment of panic disorder: Practical guidelines. Journal of Mental Health Counseling, 24(3), 224-246. 
145. Kadden, R. (2001). Behavioral and cognitive-behavioral treatments for alcoholism: research opportunities. Addictive Behaviors, 26, 489-507.

146. Monti, P.\& Rohsenow, D. (1999a). Coping skills training and cue-exposure theory in the treatment of alcoholism. Alcohol Research and Health, 23(2), 107-115.

147. Conklin, C.\& Tiffany, S. (2002). Applying extinction research and theory to cue-exposure addiction treatments. Addiction, 97, 155-167.

148. O'Farrell, T. (1991). Workshop on marital and family therapy in alcoholism treatment. in The 5th International Conference on the Treatment of Addictive Behaviours. Sydney, Australia.

149. O'Farrell, T. (1989). Marital and family therapy in alcoholism treatment. Journal of Substance Abuse Treatment, 6, 23-29.

150. Epstein, E.\& McCrady, B. (1998). Behavioral couples treatment of alcohol and drug use disorders: current status and innovations. Clinical Psychology Review, 18(6), 689-711.

151. Finfgeld, D. (2000). Use of self-help manuals to treat problem drinkers. Journal of Psychosocial Nursing and Mental Health Services, 38(4), 20-7.

152. Ryder, D., Lenton, S., Blignault, I., Hopkins, C., \& Cooke, A. (1995). The Drinker's Guide to cutting down or cutting out. Adelaide: The Drug and Alcohol Services Council.

153. Sanchez-Craig, M. (1993). Saying when: How to quit drinking or cut down. Toronto: Addiction Research Foundation.

154. Spivak, K., Sanchez-Craig, M., \& Davila, R. (1994). Assisting problem drinkers to change on their own: effect of specific and non-specific advice. Addiction, 89(9), 1135-1142.

155. Sitharthan, T., Kavanagh, D., \& Sayer, G. (1996). Moderating drinking by correspondence: an evaluation of a new method of intervention. Addiction, 91(3), 345-55.

156. Sitharthan, T., Sitharthan, G., Hough, M., \& Kavanagh, D. (1997). Cue exposure in moderation drinking: a comparison with cognitive-behavior therapy. Journal of Consulting and Clinical Psychology, 65(5), 878-882.

157. Heather, N., Richmond, R., Webster, I., Wodak, A., Hardie, M., \& Polkinghorne, H. (1989). A guide to healthier drinking. Kensington, NSW: University of New South Wales, School of Community Medicine and National Drug and Alcohol Research Centre.

158. Wilson, G. (1996). Manual-based treatments: the clinical application of research findings. Behavior Research Therapy, 34(4), 295-314.

159. Wilson, G. (1995). Manual-based treatments: the clinical application of research findings. Behavior Research Therapy, 34(4), 295-314. 
160. Connors, G., Maisto, S., \& Zywiak, W. (1996). Understanding relapse in the broader context of post-treatment functioning. Addiction, 91(Supplement), S173-S190.

161. Parks, G., Anderson, B., \& Marlatt, G. (2001a). Relapse prevention therapy, in International Handbook of Alcohol Dependence and Problems, N. Heather, T.J. Peters, T. Stockwell, Editors. John Wiley and Sons: West Sussex.

162. Larimer, M.E.P., RS, Marlatt GA. (1999). Relapse Prevention: An overview of Marlatt's cognitive-behavioral model. Alcohol Research and Health, 23(2), 151-160.

163. Miller, W., Westerberg, V., Harriis, R., \& Tonigan, J. (1996). What predicts relapse? Prospective testing of antecedent models. Addiction, 91(Suppl), S155-S172.

164. Marlatt, G. (1976). The Drinking Profile: A questionnaire for the behavioral assessment of alcoholism, in Behavior therapy assessment: Diagnosis, design, and evaluation, E.J. Mash, L.G. Terdal, Editors. Springer: New York.

165. Zywiak, W., Connors, G., Maisto, S., \& Westerberg, V. (1996). Relapse research and the Reasons for Drinking Questionnaire: a factor analysis of Marlatt's relapse taxonomy. Addiction, 91(Supplement), S121-130.

166. Annis, H. \& Graham, J. (1988). Situational confidence questionnaire (SCQ) user's guide. Addiction Research Foundation: Toronto.

167. Litman, G. (1986). Alcoholism survival: the prevention of relapse, in Treating Addictive Behaviours: Processes of Change, W.R. Miller, N.Heather, Editors. Plenum Press: New York. p. 391-405.

168. Dimeff, L. \& Marlatt, G. (1995). Relapse Prevention, in Handbook of Alcoholism Treatment Approaches: Effective Alternatives, W.M. RK Hester, Editor. Allyn \& Bacon: Massachusetts. p. 176-194.

169. Saunders, J. \& Allsop, S. (1991). Helping those who relapse., in Counselling problem drinkers, R,.Davidson, S.Rollnick, I.MacEwah, Editors Tavistock/Routledge: London.

170. Besson, J., Aeby, F., Kasas, A., Lehert, P., \& Potgieter, A. (1998). Combined efficacy of acamprosate and disulfiram in the treatment of alcoholism: A controlled study. Alcoholism, Clinical and Experimental Research, 22(3), 573-579.

171. Kiefer, F. Wiedemann, K. (2002). Combining acamprosate and naltrexone in the treatment of alcoholism, results from a randomized placebo controlled trial. European Psychiatry, 17(Suppl 1), 2.

172. Volpicelli, J., Rhines, K., Volpicelli, L., Alterman, A., \& O’Brien, C. (1997). Naltrexone and alcohol dependence. Role of subject compliance. Archives of General Psychiatry, 54(8), 737-742.

173. Kranzler, H., Modesta-Lowe, V., \& Van Kirk, J. (2000). Naltrexone vs. nefazodone for treatment of alcohol dependence. A placebo-controlled trial. Neuropsychopharmacology, 22(5), 493-503. 
174. Oncken, C., Van Kirk, J., \& Kranzler, H. (2001). Adverse effects of oral naltrexone: analysis of data from two clinical trials. Psychopharmacology, 154, 7-402.

175. O'Malley, S. (1998). Naltrexone And Alcoholism Treatmen: Treatment Improvement Protocol (TIP). Substance Abuse and Mental Health Services Administration, Center for Substance Abuse Treatment: Rockville, MD.

176. Kemp, R., Hayward, P., Applewaite, G., Everitt, B., \& David, A. (1996). Compliance therapy in psychotic patients: randomised controlled trial. British Medical Journal, 312, 5-349.

177. Reid, S., Teesson, M., Sannibale, M., Matsuda, M., \& Haber, P. (2002). The Effectiveness of Compliance Therapy in Pharmacotherapy for Alcohol Dependence: Interim Results. in The Australian Professional Society on Alcohol and Other Drugs Conference. Adelaide, South Australia.

178. Roter, D., Hall, J., Merisca, R., Nordstron, B., Cretin, D., \& Svarstad, B. (1998). Effectiveness of interventions to improve patient compliance: a meta-analysis. Medical Care, 36(8), 1138-1161.

179. Haynes, R., McDonald, H., \& Garg, A. (2002). Helping patients follow prescribed treatment: clinical applications. Journal of the American Medical Association, 288(22), 28802883.

180. Alcohol Pharmacotherapy Forum. (2001). Diagnosis and management of alcohol misuse - a guide for general practice in Australia. Intramed Pty Ltd: Sydney, Australia.

181. Latt, N., Jurd, S., Houseman, J., \& Wutzke, S. (2002). Naltrexone in alcohol dependence: a randomised controlled trial of effectiveness in a standard clinical setting. Medical Journal of Australia, 176(11), 530-534.

182. Heather, N. (1989). Disulfiram treatment for alcoholism: Deserves re-examination. British Medical Journal, 299, 471-472.

183. Brewer, C. (1996). On the specific effectiveness, and under-valuing, of pharmacological treatments for addiction: A comparison of Methadone, Naltrexone and Disulfiram with psychosocial interventions. Addiction Research, 3(4), 297-313.

184. Tonigan, J., Toscova, R., \& Miller, W. (1996). Meta-analysis of the literature on Alcoholics Anonymous: sample and study characteristics modify findings. Journal of Studies on Alcohol, 5765-72.

185. Kownacki, R. \& Shadish, W. (1999). Does Alcoholics Anonymous work? The results from a meta-analysis of controlled experiments. Substance Abuse and Misuse, 34(13), 1897-1916.

186. Longabaugh, R., Wirtz, P.W., Zweben, A., \& Stout, R.L (1998). Network support for drinking, Alcoholics Anonymous and long-term matching effects. Addiction, 93(9), 1313-33. 
187. Montgomery, H., Miller, W., \& Tonigan, J. (1995). Does Alcoholics Anonymous involvement predict treatment outcome? Journal of Substance Abuse Treatment, 12(4), 241-246.

188. Ahles, T., Schlundt, D., Prue, P., \& Rychtarik, R. (1983). Impact of aftercare arrangements on the maintenance of treatment success of abusive drinkers. Addictive Behaviors, 8, 53-58.

189. McAuliffe, W. (1990). A randomised controlled trial of recovery training and self-help for opioid addicts in New England and Hong Kong. Journal of Psychoactive Drugs, 22, 197-209.

190. McAuliffe, W. \& Ch'ien, J. (1986). Recovery training and self-help: A relapse prevention program for treated opiate addicts. Journal of Substance Abuse Treatment, 3, 9-20.

191. Spooner C, Mattick RP, \& Noffs W. (2001). Outcomes of a comprehensive treatment program for adolescents with a substance disorder. Journal of Substance Abuse Treatment, 20(3), 205-213.

192. Martin, C.\& Winters, K. (1998). Diagnosis and assessment of alcohol use disorders among adolescents. Alcohol Health and Research World, 22(2), 95-96.

193. Martin, C., Langenbucher, J., Kaczynski, N., \& Chung, T. (1996). Staging in the onset of DSM-IV alcohol symptoms in adolescents: survival/hazard analyses. Journal of Studies on Alcohol, 57(5), 549-558.

194. Winters, K., Stinchfield, R., Opland, E., Weller, C., \& Latimer, W. (2000). The effectiveness of the Minnesota Model approach in the treatment of adolescent drug abusers. Addiction, 95(4), 601-012.

195. Connie, P. \& Miller, R. (1993). Review of Dunsmore House. Western Sydney Area Health Service and the Drug and Alcohol Directorate: Mt Druitt, NSW, Australia.

196. National Health \& Medical Research Council. (1997). Clinical practice guidelines: Depression in young people. National Health \& Medical Research Council: Canberra.

197. Spooner, C., Mattick, R., \& Noffs, W. (2001). Outcomes of a comprehensive treatment program for adolescents with a substance disorder. Journal of Substance Abuse Treatment, 20(3), 205-213.

198. Rice, C., Longabaugh, R., Beattie, M., \& Noel, N. (1993). Age group differences in response to treatment for problematic alcohol use. Addiction, 88, 1369-1375.

199. Goldman, M. (1995). Recovery of Cognitive Functioning in Alcoholics: The Relationship to Treatment. Alcohol Health and Research World, 19(2), 148-154.

200. Allsop, S., Saunders, B., \& Phillips, M. (2000). The process of relapse in severely dependent male problem drinkers. Addiction, 95(1), 95-106.

201. Saunders, B., Baily, B., Phillips, M., \& Allsop, S. (1993). Women with alcohol problems: do they relapse for reasons different to their male counterparts? Addiction, 88, 14131422 .

202. Degenhardt, L, Hall, W., Teeson, M., \& Lynskey, M. (2000a). Alcohol use disorders in Australia: Findings from the National Survey of Mental Health and Well-being. National 
Drug and Alcohol Research Centre: Sydney.

203. Hulse, G., Saunders, J., Roydhouse, R., Stockwell, T., \& Basso, M. (2000). Screening for hazardous alcohol use and dependence in psychiatric in-patients using the AUDIT questionnaire. Drug and Alcohol Review, 19, 291-298.

204. Brown, R., Evans, D., Miller, I., Burgess, E., \& Mueller, T. (1997). Cognitive-behavioral treatment for depression in alcoholism. Journal of Consulting and Clinical Psychology, 65(5), 715-726.

205. Thevos, A., Roberts, J., Thomas, S., \& Randall, C. (2000). Cognitive Behavioral therapy delays relapse in female socially phobic alcoholics. Addiction Behaviors, 25(3), 333-345.

206. Barrowclough, C., Haddock, G., Tarrier, N., Lewis, S., Moring, J., O’Brien, R, Schofield, N., \& McGovern, J. (2001). Randomized controlled trial of motivational interviewing, cognitive behavior therapy, and family intervention for patients with comorbid schizophrenia and substance use disorders. American Journal of Psychiatry, 158(10), 1706-13.

207. Herman, S., Frank, K., Mowbray, C., Ribisl, K., Davidson, W., Boots-Miller, B., Jordan, L, Greenfield, A., Loveland, D., \& Luke, D. (2000). Longitudinal effects of integrated treatment on alcohol use for persons with serious mental illness and substance use disorders. Journal of Behavioral Health Services and Research, 27, 286-302.

208. Schuckit, M., Tipp, J., Bergman, M., Reich, W., Hellelbrock, V., \& Smith, T. (1997). Comparison of induced and independent major depressive disorders in 2,945 alcoholics. American Journal of Psychiatry, 154(7), 948-957.

209. Kavanagh, D.J. (2001). Treatment of comorbidity, in National Comorbidity Project, M.Teesson, L. Burns, Editors. Commonwealth Department of Health and Aged Care: Canberra. p. 60 - 70.

210. Naranjo, C., Bremner, K., \& Lanctot, K. (1995b). Effects of citalopram and a brief psycho-social intervention on alcohol intake, dependence and problems. Addiction, 90(1), 87-99.

211. Kabel, D. \& Petty, F. (1996). A placebo controlled, double-blind study of fluoxetine in severe alcohol dependence: adjunctive pharmacotherapy during and after inpatient treatment. Alcoholism: Clinical \& Experimental Research, 20(4), 780-784.

212. Kranzler, H., Burleson, J., Korner, P., Del Boca, F., Bohn, M., Brown, J., \& Liebowitz, N. (1995). Placebo-controlled trial of fluoxetine as an adjunct to relapse prevention in alcoholics. American Journal of Psychiatry, 152(3), 391-7.

213. Cornelius, J., Salloum, I., Ehler, J., Jarrett, P., Cornelius, M., Perel, J., Thase, M., \& Black, A. (1997a). Fluoxetine in depressed alcoholics. Archives of General Psychiatry, 54, 700705. 
214. Kranzler, H., Burleson, J., Del Boca, F., Babor, T., Korner, P., Brown, J., \& Bohn, M. (1994). Buspirone treatment of anxious alcoholics: a placebo-controlled trial. Archives of General Psychiatry, 51(9), 720-731.

215. Mason, B., Kocsis, J., Ritvo, E., \& Cutler, R. (1996). A double-blind, placebo-controlled trial of desipramine for primary alcohol dependence stratified on the presence or absence of major depression. JAMA, 275(10), 761-767.

216. Powell, B., Campbell, J., Landon, J., Liskow, B., Thomas, H., Nickel, E., Dale, T., Penick, E., Samuelson, S., \& Lacoursiere, R. (1995). A double-blind, placebo-controlled study of nortriptyline and bromocriptine in male alcoholics subtyped by comorbid psychiatric disorders. Alcoholism: Clinical \& Experimental Research, 19(2), 462-8.

217. Kavanagh, D., McGrath, J., Saunders, J., Dore, G., \& Clark, D. (2002). Substance misuse in patients with schizophrenia: epidemiology and management. Drugs, 62(5), 743-755.

218. Swift, W., Copeland, J., \& Hall, W. (1996). Characteristics of women with alcohol and other drug problems: findings of an Australian national survey. Addiction, 91(8), 11411150.

219. Chang, G. (2002). Brief interventions for problem drinking and women. Journal of Substance Abuse Treatment, 23, 1-7.

220. Swift, W. \& Copeland, J. (1996). Treatment needs and experiences of Australian women with alcohol and other drug problems. Drug and Alcohol Dependence, 40, 211-219.

221. Brady, M., Sibthorpe, B., Bailie, R., Ball, S., \& Sumnerdodd, P. (2002). The feasibility and acceptability of introducing brief intervention for alcohol misuse in an urban Aboriginal medical service. Drug \& Alcohol Review, 21, 375-380. 
Appendices 


\section{Appendices}

\section{Appendix 1: Safe drinking levels}

\section{FOR RISK OF HARM INTHE SHORTTERM}

\begin{tabular}{|l|l|l|l|}
\hline & $\begin{array}{l}\text { Low risk } \\
\text { (standard drinks) }\end{array}$ & $\begin{array}{l}\text { Risky } \\
\text { (standard drinks) }\end{array}$ & $\begin{array}{l}\text { High risk } \\
\text { (standard drinks) }\end{array}$ \\
\hline $\begin{array}{l}\text { Males } \\
\text { On any one day }\end{array}$ & $\begin{array}{l}\text { Up to 6 on any one } \\
\text { day, no more than 3 } \\
\text { days per week }\end{array}$ & $\begin{array}{l}7 \text { day } 10 \text { on any one } \\
\text { Females } \\
\text { On any one day }\end{array}$ & $\begin{array}{l}\text { Up to } 4 \text { on more on any } \\
\text { one day }\end{array}$ \\
\hline $\begin{array}{l}\text { day, no more than 3 } \\
\text { days a week }\end{array}$ & 5 to 6 on any one day & $\begin{array}{l}7 \text { or more on any one } \\
\text { day }\end{array}$ \\
\hline
\end{tabular}

FOR RISK OF HARM INTHE LONGTERM

\begin{tabular}{|l|l|l|l|}
\hline & $\begin{array}{l}\text { Low risk } \\
\text { (standard drinks) }\end{array}$ & $\begin{array}{l}\text { Risky } \\
\text { (standard drinks) }\end{array}$ & $\begin{array}{l}\text { High risk } \\
\text { (standard drinks) }\end{array}$ \\
\hline $\begin{array}{l}\text { Males } \\
\text { On an average day }\end{array}$ & Up to 4 per day & 5 to 6 per day & 7 or more per day \\
Overall weekly level & Up to 28 per week & 29 to 42 per week & 43 or more per week \\
\hline $\begin{array}{l}\text { Females } \\
\text { On an average day } \\
\text { Overall weekly level }\end{array}$ & $\begin{array}{l}\text { Up to 2 per day } \\
\text { Up to } 14 \text { per week }\end{array}$ & $\begin{array}{l}3 \text { to } 4 \text { per day } \\
15 \text { to } 28 \text { per week }\end{array}$ & 29 or more per week \\
\hline
\end{tabular}




\section{Appendix 2: DSM -IV diagnostic criteria for substance abuse}

A. A maladaptive pattern of substance use leading to clinically significant impairment or distress, as manifested by one (or more) of the following, occurring within a 12-month period:

a. recurrent substance use resulting in failure to fulfil major role obligations at work, school, or home.

b. recurrent substance use in situations in which it is physically hazardous (e.g. driving while intoxicated).

c. recurrent substance-related legal problems.

d. continued substance use despite having persistent or recurrent social or interpersonal problems caused or exacerbated by the effects of the substance.

B. The symptoms have not met criteria for substance dependence. 


\section{Appendix 3: DSM -IV diagnostic criteria for substance dependence}

A maladaptive pattern of substance use, leading to clinically significant impairment or distress, as manifested by three or more of the following, occurring at any time in the same 12 month period:

1. Tolerance, as defined by either:

a. a need for markedly increased amounts of the substance to achieve intoxication or the desired effect.

b. markedly diminished effect with continued use of the same amount of the substance.

2. Withdrawal, as manifested by either of the following:

a. A characteristic withdrawal syndrome

b. The same or a closely related substance is used to relieve or avoid withdrawal symptoms

3. the substance is taken in larger amounts or for a longer period than intended.

4. there is a persistent desire or unsuccessful efforts to cut down or control substance use

5. a great deal of time in spent in activities necessary to obtain the substance, use the substance, or recover from its effects.

6. important social, occupational or recreational activities are reduced or given up because of substance use.

7. substance use is continued despite knowledge of having a persistent or recurrent physical or psychological problem that is likely to have been caused or exacerbated by the substance. 